\title{
Stacks of twisted modules and integral transforms
}

\author{
Andrea D’Agnolo* $\quad$ Pietro Polesello
}

\begin{abstract}
Stacks were introduced by Grothendieck and Giraud and are, roughly speaking, sheaves of categories. Kashiwara developed the theory of twisted modules, which are objects of stacks locally equivalent to stacks of modules over sheaves of rings. In this paper we recall these notions, and we develop the formalism of operations for stacks of twisted modules. As an application, we state a twisted version of an adjunction formula which is of use in the theory of integral transforms for sheaves and $D$-modules.
\end{abstract}

2000 Mathematics Subject Classification: 14A20, 32C38, 35A22

\section{Contents}

1 Stacks of twisted modules 4

1.1 Prestacks . . . . . . . . . . . . . . . . 4

1.2 Stacks . . . . . . . . . . . . . . . . . . 6

1.3 Constructions of stacks . . . . . . . . . . . . . . . 8

1.4 Operations . . . . . . . . . . . . . . . . . . . 10

1.5 Linear stacks . . . . . . . . . . . . . . . . . . . . . . 10

1.6 Operations . . . . . . . . . . . . . . . . . . . . . . . 11

1.7 Stacks of twisted modules . . . . . . . . . . . . . . . . . . . 12

2 Operations 13

2.1 Morita theorv I. Functors admitting an adjoint . . . . . . . . . . . 13

2.2 Internal product of stacks of twisted modules . . . . . . . . . . 15

2.3 Morita theorv II. Relative case . . . . . . . . . . . . . . . . 17

2.4 Pull-back of stacks of twisted modules . . . . . . . . . . . . 18

2.5 Twisted sheaf-theoretical operations . . . . . . . . . . . . 20

2.6 Derived twisted operations . . . . . . . . . . . . . . . 21

*A.D'A. had the occasion to visit the Research Institute for Mathematical Sciences of Kyoto University during the preparation of this paper. Their warm hospitality is gratefully acknowledged.

${ }_{\ddagger}^{\ddagger}$ P.P. was partially supported by INdAM during the preparation of this paper 
3 Descent 22

3.1 Morita theorv III. Equivalences . . . . . . . . . . . . . . . . . . . . 23

3.2 Twisting data on an open covering . . . . . . . . . . . . . . . . 24

3.3 Twisting data . . . . . . . . . . . . . . . . . . . . 24

3.4 Classification of stacks of twisted modules . . . . . . . . . . . . . . 29

3.5 Operations in terms of twisting data . . . . . . . . . . . . . . . . . 30

3.6 Complex powers of line bundles . . . . . . . . . . . . . . . . 31

$4 \quad$ Examples and applications $\quad 32$

4.1 Twisted modules over commutative local rings . . . . . . . . . . . 32

4.2 Twisting by inner forms . . . . . . . . . . . . . . . . . . . . . . 34

4.3 Azumava algebras . . . . . . . . . . . . . . . . . . . 35

4.4 Twisted differential operators . . . . . . . . . . . . . . . . . . . . . 37

4.5 Twisted $\mathcal{D}$-module operations . . . . . . . . . . . . . . . . . . . . . 39

4.6 Twisted adjunction formula . . . . . . . . . . . . . . . . . . . . 40

\section{Introduction}

Stacks are, roughly speaking, sheaves of categories. They were introduced by Grothendieck and Giraud [14] in algebraic geometry where some special stacks, called gerbes, are now commonly used in moduli problems to describe objects with automorphisms (see for example 2, 29]). Recently, gerbes have infiltrated differential geometry and mathematical physics (see for example [7, 31, 15, 6]).

We are interested here in the related notion of twisted modules, which are objects of stacks locally equivalent to stacks of modules over sheaves of rings. The simplest example is that of stacks of twisted $\mathcal{R}$-modules on a locally ringed space $(X, \mathcal{R})$. These can be considered as higher cohomological analogues to line bundles. More precisely, line bundles on $X$ are sheaves of $\mathcal{R}$-modules locally isomorphic to $\mathcal{R}$, and their isomorphism classes describe the cohomology group $H^{1}\left(X ; \mathcal{R}^{\times}\right)$. Stacks of twisted $\mathcal{R}$-modules are $\mathcal{R}$-linear stacks on $X$ locally equivalent to the stack $\mathfrak{M o d}(\mathcal{R})$ of $\mathcal{R}$-modules and, as we shall recall, their equivalence classes describe the cohomology group $H^{2}\left(X ; \mathcal{R}^{\times}\right)$. As line bundles correspond to principal $\mathcal{R}^{\times}$-bundles, so stacks of twisted $\mathcal{R}$-modules correspond to gerbes with band $\mathcal{R}^{\times}$. However, this correspondence no longer holds for the more general type of stacks of twisted modules that we consider here.

Twisted modules appear in works by Kashiwara on representation theory [18] and on quantization [20]. In the first case, they were used to describe solutions on flag manifolds to quasi-equivariant modules over rings of twisted differential operators (see also [26]). In the second case, twisted modules turned out to be the natural framework for a global study of microdifferential systems on a holomorphic contact manifold (see also [28, 30]). Rings of microdifferential operators can be locally defined on a contact manifold, but do not necessarily exist globally. Kashi- 
wara proved that there exists a globally defined $\mathbb{C}$-linear stack which is locally equivalent to the stack of modules over a ring of microdifferential operators.

Twisted modules induced by Azumaya algebras are used in [8] 11] in relation with the Fourier-Mukai transform.

Motivated by Kashiwara's work on quantization, we consider here twisted modules over sheaves of rings which are not necessarily commutative nor globally defined. More precisely, let $X$ be a topological space, or more generally a site, and $\mathcal{R}$ a sheaf of commutative rings on $X$. Then $\mathfrak{M}$ is a stack of $\mathcal{R}$-twisted modules on $X$ if it is $\mathcal{R}$-linear and there exist an open covering $X=\bigcup_{i \in I} U_{i}$, sheaves of $\left.\mathcal{R}\right|_{U_{i}}$-algebras $\mathcal{A}_{i}$, and $\left.\mathcal{R}\right|_{U_{i}}$-equivalences $\left.\mathfrak{M}\right|_{U_{i}} \approx \mathfrak{M} \mathfrak{o d}\left(\mathcal{A}_{i}\right)$, where $\mathfrak{M o d}\left(\mathcal{A}_{i}\right)$ denotes the stack of left $\mathcal{A}_{i}$-modules on $U_{i}$. We review the notions of stack and stack of twisted modules in Section 1 restricting to the case of topological spaces for simplicity of exposition.

Morita theory is the basic tool to deal with stacks of $\mathcal{R}$-twisted modules, and we use it to develop the formalism of operations, namely duality $(\cdot)^{\circledast-1}$, internal product $\circledast_{\mathcal{R}}$, and inverse image $f^{\circledast}$ by a continuous map $f: Y \rightarrow X$. If $\mathcal{A}$ and $\mathcal{A}^{\prime}$ are sheaves of $\mathcal{R}$-algebras on $X$, these operations satisfy $\mathfrak{M o d}(\mathcal{A})^{\circledast-1} \approx \mathfrak{M} \mathfrak{o d}\left(\mathcal{A}^{\text {op }}\right)$, $\mathfrak{M o d}(\mathcal{A}) \circledast_{\mathcal{R}} \mathfrak{M o d}\left(\mathcal{A}^{\prime}\right) \approx \mathfrak{M o d}\left(\mathcal{A} \otimes_{\mathcal{R}} \mathcal{A}^{\prime}\right)$, and $f^{\circledast} \mathfrak{M o d}(\mathcal{A}) \approx \mathfrak{M o d}\left(f^{-1} \mathcal{A}\right)$. With this formalism at hand, we then describe Grothendieck's six operations for derived categories of twisted modules over locally compact Hausdorff topological spaces. This is the content of Section 2

Morita theory is used again in Section 3 to describe effective descent data attached to semisimplicial complexes. In particular, we get a Cech-like classification of stacks of $\mathcal{R}$-twisted modules, with invertible bimodules as cocycles, which is parallel to the bitorsor description of gerbes in [6].

In Section 4 assuming that $\mathcal{R}$ is a commutative local ring, we recall the above mentioned classification of stacks of twisted $\mathcal{R}$-modules in terms of $H^{2}\left(X ; \mathcal{R}^{\times}\right)$. We then consider the case of twisted modules induced by ordinary modules over an inner form of a given $\mathcal{R}$-algebra. This allows us to present in a unified manner the examples provided by modules over Azumaya algebras and over rings of twisted differential operators. Finally, we state a twisted version of an adjunction formula for sheaves and $D$-modules, which is of use in the theory of integral transforms with regular kernel, as the Radon-Penrose transform.

This paper is in part a survey and in part original. The survey covers material from Kashiwara's papers [18, 20, from his joint works [26, 27, and from the last chapter of his forthcoming book with Pierre Schapira [25. The main original contribution is in establishing the formalism of operations for stacks of twisted modules.

It is a pleasure to thank Masaki Kashiwara for several useful discussions and insights. We also wish to thank him and Pierre Schapira for allowing us to use results from a preliminary version of their book 25. 


\section{Stacks of twisted modules}

The theory of stacks is due to Grothendieck and Giraud 14. We review it here restricting for simplicity to the case of stacks on topological spaces (thus avoiding the notions of site and of fibered category). Finally, we recall the notion of stack of twisted modules, considering the case of modules over rings which are not necessarily commutative nor globally defined. Our main references were [18, 20, 24, 25].

\subsection{Prestacks.}

We assume that the reader is familiar with the basic notions of category theory, as those of category, functor between categories, transformation between functors (also called morphism of functors), and equivalence of categories.

If $\mathrm{C}$ is a category, we denote by $\mathrm{Ob}(\mathrm{C})$ the $\operatorname{set}^{1}$ of its objects, and by $\operatorname{Hom}_{\mathrm{C}}(c, d)$ the set of morphisms between the objects $c$ and $d$. The identity of $\operatorname{Hom}_{C}(c, c)$ will be denoted by $\mathrm{id}_{c}$.

Denote by $C^{\mathrm{op}}$ the opposite category, which has the same objects as $\mathrm{C}$ and reversed morphisms $\operatorname{Hom}_{\mathrm{Cop}}(c, d)=\operatorname{Hom}_{\mathrm{C}}(d, c)$. If $\mathrm{D}$ is another category, denote by $\operatorname{Hom}(C, D)$ the category of functors from $C$ to $D$, with transformations as morphisms.

Let $X$ be a topological space, and denote by $\mathrm{X}$ the category of its open subsets with inclusion morphisms. Recall that the category of presheaves on $X$ with values in a category $C$ is the category $\operatorname{Hom}\left(X^{o p}, C\right)$ of contravariant functors from $X$ to C. In particular, presheaves of sets are obtained by taking $\mathrm{C}=$ Set, the category of $\operatorname{sets}^{2}$ and maps of sets.

Considering $\mathrm{C}=\mathrm{Cat}$, the category of categories ${ }^{3}$ and functors, one has a notion of presheaf of categories. This a functor $\mathcal{F}: \mathrm{X}^{\mathrm{op}} \rightarrow$ Cat, and if $W \stackrel{v}{\rightarrow} V \stackrel{u}{\rightarrow} U$ are inclusions of open sets, the restriction functors $\mathcal{F}(u): \mathcal{F}(U) \rightarrow \mathcal{F}(V)$ and $\mathcal{F}(v): \mathcal{F}(V) \rightarrow \mathcal{F}(W)$ are thus required to satisfy the equality $\mathcal{F}(v) \circ \mathcal{F}(u)=$ $\mathcal{F}(u \circ v)$. Such a requirement is often too strong in practice, and the notion of prestack is obtained by weakening this equality to an isomorphism of functors, i.e. to an invertible transformation.

\footnotetext{
${ }^{1}$ Following Bourbaki's appendix in SGA4, one way to avoid the paradoxical situation of dealing with the set of all sets is to consider universes, which are "big" sets of sets stable by most of the set-theoretical operations. We assume here to be given a universe $\mathcal{U}$ and, unless otherwise stated, all categories $\mathrm{C}$ will be assumed to be $\mathcal{U}$-categories, i.e. categories such that $\mathrm{Ob}(\mathrm{C}) \subset \mathcal{U}$ and $\operatorname{Hom}_{\mathrm{C}}(c, d) \in \mathcal{U}$ for every pair of objects.

${ }^{2}$ More precisely, Set denotes the $\mathcal{U}$-category of sets belonging to the fixed universe $\mathcal{U}$.

${ }^{3}$ More precisely, let $\mathcal{V}$ be another fixed universe with $\mathcal{U} \in \mathcal{V}$. Then Cat denotes the $\mathcal{V}$-category whose objects are $\mathcal{U}$-categories. From now on we will leave to the reader who feels that need the task of making the universes explicit.
} 
In other words, prestacks are the 2 -categorical ${ }^{4}$ version of presheaves of categories. However, we prefer not to use the language of 2-categories, giving instead the unfolded definition of prestack.

Definition 1.1. A prestack $\mathfrak{P}$ on $X$ consists of the data

(a) for every open subset $U \subset X$, a category $\mathfrak{P}(U)$,

(b) for every inclusion $V \stackrel{u}{\rightarrow} U$ of open subsets, a functor $\mathfrak{P}(u): \mathfrak{P}(U) \rightarrow \mathfrak{P}(V)$, called restriction functor, and

(c) for every inclusion $W \stackrel{v}{\rightarrow} V \stackrel{u}{\rightarrow} U$ of open subsets, invertible transformations $\mathfrak{P}(v, u): \mathfrak{P}(v) \circ \mathfrak{P}(u) \Rightarrow \mathfrak{P}(u \circ v)$ of functors from $\mathfrak{P}(U)$ to $\mathfrak{P}(W)$,

subject to the conditions

(i) if $U$ is an open subset, then $\mathfrak{P}\left(\operatorname{id}_{U}\right)=\operatorname{id}_{\mathfrak{P}(U)}$ and $\mathfrak{P}\left(\operatorname{id}_{U}, \mathrm{id}_{U}\right)=\operatorname{id}_{\operatorname{id}_{\mathfrak{P}(U)}}$;

(ii) if $Y \stackrel{w}{\rightarrow} W \stackrel{v}{\rightarrow} V \stackrel{u}{\rightarrow} U$ are inclusions of open subsets, then the following diagram of functors from $\mathfrak{P}(U)$ to $\mathfrak{P}(Y)$ commutes

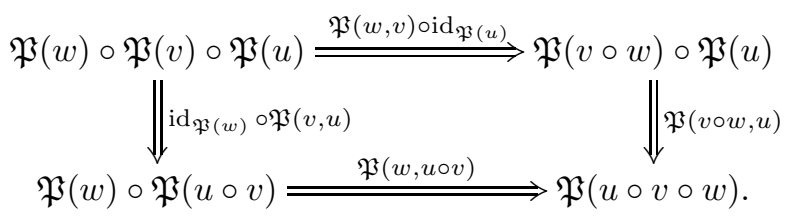

In particular, $\mathfrak{P}\left(u, \mathrm{id}_{U}\right)=\mathfrak{P}\left(\operatorname{id}_{V}, u\right)=\operatorname{id}_{\mathfrak{P}(u)}$.

For $\mathcal{F} \in \mathfrak{P}(U)$ and $V \stackrel{u}{\rightarrow} U$ an open inclusion, one usually writes $\left.\mathcal{F}\right|_{V}$ instead of $\mathfrak{P}(u)(\mathcal{F})$. One denotes by $\left.\mathfrak{P}\right|_{U}$ the natural restriction of $\mathfrak{P}$ to $U$ given by $V \mapsto \mathfrak{P}(V)$ for $V \subset U$.

Definition 1.2. Let $\mathfrak{P}$ and $\mathfrak{Q}$ be prestacks on $X$. A functor of prestacks $\varphi: \mathfrak{P} \rightarrow$ $\mathfrak{Q}$ consists of the data

(a) for any open subset $U \subset X$, a functor $\varphi(U): \mathfrak{P}(U) \rightarrow \mathfrak{Q}(U)$,

\footnotetext{
${ }^{4}$ Roughly speaking, a 2-category (refer to 33 §9] for details) $\mathbf{C}$ is a "category enriched in Cat", i.e. a category whose morphism sets are the object sets of categories $\operatorname{Hom}_{\mathbf{C}}(c, d)$, such that composition is a functor. Morphisms in the category $\operatorname{Hom}_{\mathbf{C}}(c, d)$ are called 2-cells. The basic example is the 2-category Cat which has categories as objects, functors as morphisms, and transformations as 2-cells.

There is a natural notion of pseudo-functor between 2-categories, preserving associativity for the composition functor only up to an invertible 2-cell. Then a prestack (see [SGA1 exposé VI]) is a pseudo-functor $\mathbf{X}^{\mathrm{op}} \rightarrow \mathbf{C a t}$, where $\mathbf{X}^{\mathrm{op}}$ is the 2-category obtained by trivially enriching $\mathbf{X}^{\mathrm{op}}$ with identity 2-cells. Functors of prestacks and their transformations are transformations and modifications of pseudo-functors, respectively.

Note that Corollary 9.2 of [33] asserts that any prestack is equivalent, in the 2-category of pseudo-functors, to a presheaf of categories. However, this equivalence is not of practical use for our purposes.
} 
(b) for any open inclusion $V \stackrel{u}{\rightarrow} U$, an invertible transformation $\varphi(u): \varphi(V) \circ$ $\mathfrak{P}(u) \Rightarrow \mathfrak{Q}(u) \circ \varphi(U)$ of functors from $\mathfrak{P}(U)$ to $\mathfrak{Q}(V)$,

subject to the condition

(i) if $W \stackrel{v}{\rightarrow} V \stackrel{u}{\rightarrow} U$ are inclusions of open subsets, then the following diagram of functors from $\mathfrak{P}(U)$ to $\mathfrak{Q}(W)$ commutes

$$
\begin{gathered}
\varphi(W) \circ \mathfrak{P}(v) \circ \mathfrak{P}(u) \stackrel{\varphi(v) \circ \operatorname{id}_{\mathfrak{P}(u)}}{=} \mathfrak{Q}(v) \circ \varphi(V) \circ \mathfrak{P}(u) \stackrel{\operatorname{id}_{\mathfrak{Q}(v)} \circ \varphi(u)}{=} \mathfrak{Q}(v) \circ \mathfrak{Q}(u) \circ \varphi(U) \\
\| \operatorname{id}_{\varphi(W)} \circ \mathfrak{P}(v, u) \\
\varphi\left(w(v, u) \circ \operatorname{id}_{\varphi(U)} \|\right.
\end{gathered}
$$

In particular, $\varphi\left(\operatorname{id}_{U}\right)=\operatorname{id}_{\varphi(U)}$.

Definition 1.3. Let $\varphi, \psi: \mathfrak{P} \rightarrow \mathfrak{Q}$ be functors of prestacks. A transformation $\alpha: \varphi \Rightarrow \psi$ of functors of prestacks consists of the data

(a) for any open subset $U \subset X$, a transformation $\alpha(U): \varphi(U) \Rightarrow \psi(U)$ of functors from $\mathfrak{P}(U)$ to $\mathfrak{Q}(U)$,

such that

(i) if $V \stackrel{u}{\rightarrow} U$ is an inclusion of open subsets, then the following diagram of functors from $\mathfrak{P}(U)$ to $\mathfrak{Q}(V)$ commutes

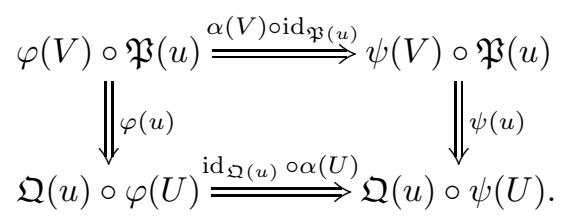

An example of prestack is the prestack $\mathfrak{P S h _ { X }}$ of presheaves of sets on $X$. It associates to an open subset $U \subset X$ the category Hom ( $U^{\text {op }}$, Set) of presheaves of sets on $U$, and to an open inclusion $V \subset U$ the restriction functor $\mathfrak{P S h}_{X}(U) \rightarrow$ $\mathfrak{P S \mathfrak { h } _ { X }}(V),\left.\mathcal{F} \mapsto \mathcal{F}\right|_{V}$. For open inclusions $W \subset V \subset U$ one has $\left.\left.\mathcal{F}\right|_{V}\right|_{W}=\left.\mathcal{F}\right|_{W}$, so that $\mathfrak{P} \mathfrak{S h}_{X}$ is in fact a presheaf of categories.

If $\mathfrak{P}$ and $\mathfrak{Q}$ are prestacks, one gets another prestack $\mathfrak{H o m}(\mathfrak{P}, \mathfrak{Q})$ by associating to an open subset $U \subset X$ the category $\operatorname{Hom}\left(\left.\mathfrak{P}\right|_{U},\left.\mathfrak{Q}\right|_{U}\right)$ of functors of prestacks from $\left.\mathfrak{P}\right|_{U}$ to $\left.\mathfrak{Q}\right|_{U}$, with transformations of functors of prestacks as morphisms, and with the natural restriction functors. Note that $\mathfrak{H o m}(\mathfrak{P}, \mathfrak{Q})$ is actually a presheaf of categories.

(Pre)stacks which are not (pre)sheaves of categories will appear in Section 2

\subsection{Stacks.}

The analogy between presheaves and prestacks goes on for sheaves and stacks. 
Let $X$ be a topological space. Given a family of subsets $\left\{U_{i}\right\}_{i \in I}$ of $X$, let us use the notations

$$
U_{i j}=U_{i} \cap U_{j}, \quad U_{i j k}=U_{i} \cap U_{j} \cap U_{k}, \quad \text { etc. }
$$

Recall that a presheaf of sets $\mathcal{F}$ on $X$ is called a sheaf if for any open subset $U \subset X$, and any open covering $\left\{U_{i}\right\}_{i \in I}$ of $U$, the natural sequence given by the restriction maps

$$
\mathcal{F}(U) \longrightarrow \prod_{i \in I} \mathcal{F}\left(U_{i}\right) \Longrightarrow \prod_{i, j \in I} \mathcal{F}\left(U_{i j}\right)
$$

is exact, i.e. if for any family of sections $s_{i} \in \mathcal{F}\left(U_{i}\right)$ satisfying $\left.s_{i}\right|_{U_{i j}}=\left.s_{j}\right|_{U_{i j}}$ there is a unique section $s \in \mathcal{F}(U)$ such that $\left.s\right|_{U_{i}}=s_{i}$.

Similarly to the definition of sheaf, a prestack $\mathfrak{S}$ on $X$ is called a stack if for any open subset $U \subset X$, and any open covering $\left\{U_{i}\right\}_{i \in I}$ of $U$, the natural sequence given by the restriction functors

$$
\mathfrak{S}(U) \longrightarrow \prod_{i \in I} \mathfrak{S}\left(U_{i}\right) \Longrightarrow \prod_{i, j \in I} \mathfrak{S}\left(U_{i j}\right) \Longrightarrow \prod_{i, j, k \in I} \mathfrak{S}\left(U_{i j k}\right)
$$

is exact in the sense of [SGA1, exposé XIII], i.e. if the category $\mathfrak{S}(U)$ is equivalent to the category whose objects are families of objects $\mathcal{F}_{i}$ of $\mathfrak{S}\left(U_{i}\right)$ and of isomorphisms $\theta_{i j}:\left.\left.\mathcal{F}_{j}\right|_{U_{i j}} \stackrel{\sim}{\rightarrow} \mathcal{F}_{i}\right|_{U_{i j}}$ which are compatible in the triple intersections, in a natural sense.

More explicitly, recall that a descent datum for $\mathfrak{S}$ on $U$ is a triplet

$$
F=\left(\left\{U_{i}\right\}_{i \in I},\left\{\mathcal{F}_{i}\right\}_{i \in I},\left\{\theta_{i j}\right\}_{i, j \in I}\right),
$$

where $\left\{U_{i}\right\}_{i \in I}$ is an open covering of $U, \mathcal{F}_{i} \in \mathfrak{S}\left(U_{i}\right)$, and $\theta_{i j}:\left.\left.\mathcal{F}_{j}\right|_{U_{i j}} \stackrel{\sim}{\rightarrow} \mathcal{F}_{i}\right|_{U_{i j}}$ are isomorphisms such that the following diagram of isomorphisms commutes

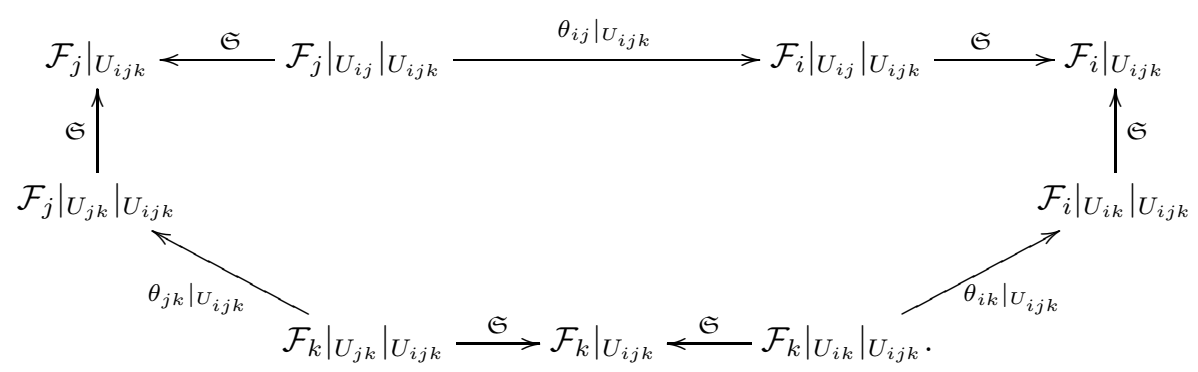

The descent datum $F$ is called effective if there exist $\mathcal{F} \in \mathfrak{S}(U)$ and isomorphisms $\theta_{i}:\left.\mathcal{F}\right|_{U_{i}} \stackrel{\sim}{\rightarrow} \mathcal{F}_{i}$ for each $i$, such that the following diagram of isomorphisms commutes

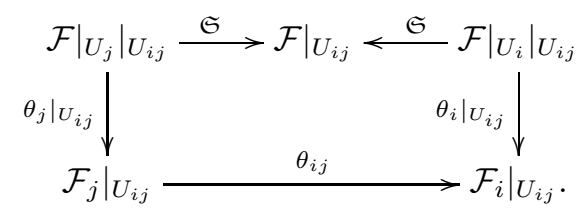


To $\mathfrak{S}$ a prestack on $X$ is attached a bifunctor of prestacks

$$
\mathcal{H o m}_{\mathfrak{S}}: \mathfrak{S}^{\text {op }} \times \mathfrak{S} \rightarrow \mathfrak{P S}_{X},
$$

associating to $\mathcal{F}, \mathcal{G} \in \mathfrak{S}(U)$ the presheaf of sets on $U \subset X$ given by

$$
\mathcal{H o m}_{\left.\mathfrak{S}\right|_{U}}(\mathcal{F}, \mathcal{G}): V \mapsto \operatorname{Hom}_{\mathfrak{S}(V)}\left(\left.\mathcal{F}\right|_{V},\left.\mathcal{G}\right|_{V}\right)
$$

Definition 1.4. (i) A prestack $\mathfrak{S}$ on $X$ is called separated if for any open subset $U$, and any $\mathcal{F}, \mathcal{G} \in \mathfrak{S}(U)$, the presheaf $\mathcal{H o m}_{\left.\mathfrak{S}\right|_{U}}(\mathcal{F}, \mathcal{G})$ is a sheaf.

(ii) A stack is a separated prestack such that any descent datum is effective.

(iii) Functors and transformations of stacks are functors and transformations of the underlying prestacks, respectively.

For example, the prestack $\mathfrak{S h}_{X}$ of sheaves of sets, associating to $U \subset X$ the category of sheaves of sets on $U$, is actually a stack. As another example, if $\mathfrak{S}$ and $\mathfrak{T}$ are stacks, then the prestack $\mathfrak{H o m}(\mathfrak{S}, \mathfrak{T})$ is a stack. (Note that both $\mathfrak{S h}_{X}$ and $\mathfrak{H o m}(\mathfrak{S}, \mathfrak{T})$ are in fact sheaves of categories.)

One says that a functor of stacks $\varphi: \mathfrak{S} \rightarrow \mathfrak{T}$ is an equivalence if there exists a functor $\psi: \mathfrak{T} \rightarrow \mathfrak{S}$, called a quasi-inverse to $\varphi$, and invertible transformations $\varphi \circ \psi \Rightarrow \mathrm{id}_{\mathfrak{T}}$ and $\psi \circ \varphi \Rightarrow \mathrm{id}_{\mathfrak{S}}$. One says that $\varphi$ admits a right adjoint if there exists a functor of stacks $\psi: \mathfrak{T} \rightarrow \mathfrak{S}$, called a right adjoint to $\varphi$, and an invertible transformation $\mathcal{H o m}_{\mathfrak{T}}(\varphi(\cdot), \cdot) \Rightarrow \mathcal{H o m}_{\mathfrak{S}}(\cdot, \psi(\cdot))$. Similarly for left adjoint. Finally, if $\mathfrak{T}=\mathfrak{S h}_{X}$, one says that $\varphi: \mathfrak{S} \rightarrow \mathfrak{S h}_{X}$ is representable if there exists $\mathcal{F} \in \mathfrak{S}(X)$, called a representative of $\varphi$, and an invertible transformation $\varphi \Rightarrow \mathcal{H o m}_{\mathfrak{S}}(\mathcal{F}, \cdot)$.

Lemma 1.5. For a functor of stacks to be an equivalence (resp. to admit a right or left adjoint, resp. to be representable) is a local property.

Proof. Right or left adjoints and representatives are unique up to unique isomorphisms, and hence glue together globally. As for equivalences, assume that $\varphi$ is locally an equivalence. Then we have to show that for each open subset $U \subset X$ the functors $\varphi(U)$ are fully faithful and essentially surjective. Being fully faithful is a local property already for separated prestacks. Assume that $\varphi\left(U_{i}\right)$ are essentially surjective for a covering $U=\bigcup_{i} U_{i}$. Let $\mathcal{G} \in \mathrm{Ob}(\mathfrak{T}(U))$, and choose $\mathcal{F}_{i} \in \mathrm{Ob}\left(\mathfrak{S}\left(U_{i}\right)\right)$ with isomorphisms $\left.\varphi\left(U_{i}\right)\left(\mathcal{F}_{i}\right) \stackrel{\sim}{\longrightarrow} \mathcal{G}\right|_{U_{i}}$. Since $\varphi$ is fully faithful, the restriction morphisms of $\left.\mathcal{G}\right|_{U_{i}}$ give descent data for $\mathcal{F}_{i}$. Finally, since $\mathfrak{S}$ is a stack, one gets $\mathcal{F} \in \mathrm{Ob}(\mathfrak{S}(U))$ with $\varphi(U)(\mathcal{F}) \stackrel{\sim}{\longrightarrow} \mathcal{G}$.

\subsection{Constructions of stacks.}

The forgetful functor, associating to a sheaf of sets its underlying presheaf, has a left adjoint, associating a sheaf $\mathcal{P}^{+}$to a presheaf $\mathcal{P}$. There is a similar construction associating a stack $\mathfrak{P}^{+}$to a prestack $\mathfrak{P}$. This is done in two steps as 
follows. Consider first the separated prestack $\mathfrak{P}^{a}$, with the same objects as $\mathfrak{P}$ and morphisms

$$
\operatorname{Hom}_{\mathfrak{P}^{a}(U)}(\mathcal{F}, \mathcal{G})=\Gamma\left(U ; \mathcal{H} o m_{\left.\mathfrak{P}\right|_{U}}^{+}(\mathcal{F}, \mathcal{G})\right)
$$

Then let $\mathfrak{P}^{+}(U)$ be the category whose objects are descent data for $\mathfrak{P}^{a}$ on $U$, and whose morphisms $\left(\left\{U_{i}\right\},\left\{\mathcal{F}_{i}\right\},\left\{\theta_{i i^{\prime}}\right\}\right) \rightarrow\left(\left\{V_{j}\right\},\left\{\mathcal{G}_{j}\right\},\left\{\varpi_{j j^{\prime}}\right\}\right)$ consist of morphisms $\varphi_{j i}:\left.\left.\mathcal{F}_{i}\right|_{U_{i} \cap V_{j}} \rightarrow \mathcal{G}_{j}\right|_{U_{i} \cap V_{j}}$ such that $\varpi_{j^{\prime} j} \circ \varphi_{j i}=\varphi_{j^{\prime} i^{\prime}} \circ \theta_{i^{\prime} i}$ on $U_{i i^{\prime}} \cap V_{j j^{\prime}}$.

Since sheaves of sets form a stack, descent data for sheaves are effective. Similarly, it is possible to patch stacks together. More precisely, a descent datum for stacks on $X$ is a quadruplet

$$
S=\left(\left\{U_{i}\right\}_{i \in I},\left\{\mathfrak{S}_{i}\right\}_{i \in I},\left\{\varphi_{i j}\right\}_{i, j \in I},\left\{\alpha_{i j k}\right\}_{i, j, k \in I}\right)
$$

where $\left\{U_{i}\right\}_{i \in I}$ is an open covering of $X, \mathfrak{S}_{i}$ are stacks on $U_{i}, \varphi_{i j}:\left.\left.\mathfrak{S}_{j}\right|_{U_{i j}} \stackrel{\approx}{\rightarrow} \mathfrak{S}_{i}\right|_{U_{i j}}$ are equivalences of stacks, and $\alpha_{i j k}: \varphi_{i j} \circ \varphi_{j k} \Rightarrow \varphi_{i k}$ are invertible transformations of functors from $\left.\mathfrak{S}_{k}\right|_{U_{i j k}}$ to $\left.\mathfrak{S}_{i}\right|_{U_{i j k}}$, such that for any $i, j, k, l \in I$, the following diagram of transformations of functors from $\left.\mathfrak{S}_{l}\right|_{U_{i j k l}}$ to $\left.\mathfrak{S}_{i}\right|_{U_{i j k l}}$ commutes

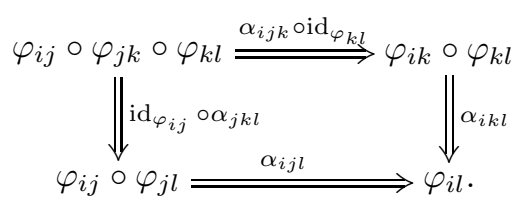

Proposition 1.6. Descent data for stacks are effective, meaning that given a descent datum for stacks $S$ as in (1.2), there exist a stack $\mathfrak{S}$ on $X$, equivalences of stacks $\varphi_{i}:\left.\mathfrak{S}\right|_{U_{i}} \stackrel{\approx}{\rightarrow} \mathfrak{S}_{i}$, and invertible transformations of functors $\alpha_{i j}: \varphi_{i j} \circ$ $\left.\left.\varphi_{j}\right|_{U_{i j}} \Rightarrow \varphi_{i}\right|_{U_{i j}}$ such that $\left.\left.\alpha_{i j}\right|_{U_{i j k}} \circ \alpha_{j k}\right|_{U_{i j k}}=\left.\alpha_{i k}\right|_{U_{i j k}} \circ \alpha_{i j k}$. The stack $\mathfrak{S}$ is unique up to equivalence.

Sketch of proof. For $U \subset X$ open, denote by $\mathfrak{S}(U)$ the category whose objects are triplets

$$
F=\left(\left\{V_{i}\right\}_{i \in I},\left\{\mathcal{F}_{i}\right\}_{i \in I},\left\{\xi_{i j}\right\}_{i, j \in I}\right)
$$

where $V_{i}=U \cap U_{i}, \mathcal{F}_{i} \in \mathrm{Ob}\left(\mathfrak{S}_{i}\left(V_{i}\right)\right)$, and $\xi_{i j}:\left.\varphi_{i j}\left(\left.\mathcal{F}_{j}\right|_{V_{i j}}\right) \rightarrow \mathcal{F}_{i}\right|_{V_{i j}}$ are isomorphisms such that for $i, j, k \in I$ the following diagram commutes

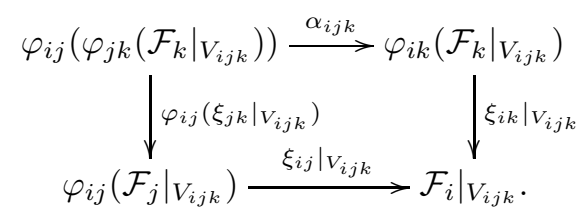


For $G=\left(\left\{V_{i}\right\},\left\{\mathcal{G}_{i}\right\},\left\{\eta_{i j}\right\}\right)$, a morphisms $\gamma: F \rightarrow G$ in $\mathfrak{S}(U)$ consists of morphisms $\gamma_{i}: \mathcal{F}_{i} \rightarrow \mathcal{G}_{i}$ in $\mathfrak{S}_{i}\left(V_{i}\right)$ such that the following diagram commutes

$$
\begin{gathered}
\left.\varphi_{i j}\left(\left.\mathcal{F}_{j}\right|_{V_{i j}}\right) \stackrel{\xi_{i j}}{\longrightarrow} \mathcal{F}_{i}\right|_{V_{i j}} \\
\qquad \varphi_{i j}\left(\left.\gamma_{j}\right|_{V_{i j}}\right) \\
\left.\varphi_{i j}\left(\left.\mathcal{G}_{j}\right|_{V_{i j}}\right) \stackrel{\left.\eta_{i j}\right|_{V_{i j}}}{\longrightarrow} \mathcal{G}_{i}\right|_{V_{i j}} .
\end{gathered}
$$

Then one checks that the prestack $\mathfrak{S}: U \rightarrow \mathfrak{S}(U)$ is a stack satisfying the requirements in the statement.

\subsection{Operations.}

Let us recall the stack-theoretical analogue of internal and external operations for sheaves.

Given two stacks $\mathfrak{S}$ and $\mathfrak{S}^{\prime}$ on $X$, denote by $\mathfrak{S} \times \mathfrak{S}^{\prime}$ the prestack $\mathfrak{S} \times \mathfrak{S}^{\prime}(U)=$ $\mathfrak{S}(U) \times \mathfrak{S}^{\prime}(U)$. This is actually a stack. We already noticed that the prestack $\mathfrak{H o m}\left(\mathfrak{S}, \mathfrak{S}^{\prime}\right)$ is a stack. If $\mathfrak{S}^{\prime \prime}$ is another stack, there is a natural equivalence

$$
\mathfrak{H o m}\left(\mathfrak{S} \times \mathfrak{S}^{\prime}, \mathfrak{S}^{\prime \prime}\right) \stackrel{\approx}{\rightarrow} \mathfrak{H o m}\left(\mathfrak{S}, \mathfrak{H o m}\left(\mathfrak{S}^{\prime}, \mathfrak{S}^{\prime \prime}\right)\right) \text {. }
$$

Let $f: Y \rightarrow X$ be a continuous map of topological spaces. If $\mathfrak{T}$ is a stack on $Y$, denote by $f_{*} \mathfrak{T}$ the prestack $f_{*} \mathfrak{T}(U)=\mathfrak{T}\left(f^{-1} U\right)$, which is actually a stack. If $\mathfrak{S}$ is a stack on $X$, denote by $f^{-1} \mathfrak{S}=\left(f^{\sim} \mathfrak{S}\right)^{+}$the stack associated with the prestack $f^{\sim \mathfrak{S}}$ defined as follows. For $V \subset Y, f^{\sim} \mathfrak{S}(V)$ is the category whose objects are the disjoint union $\bigsqcup_{U: f^{-1} U \supset V} \mathrm{Ob}(\mathfrak{S}(U))$, and whose morphisms are given by

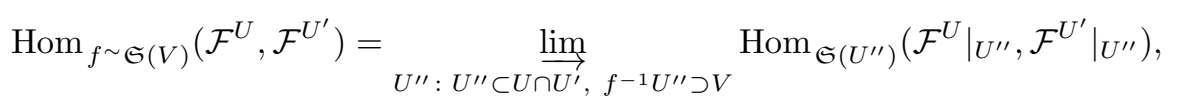

for $\mathcal{F}^{U} \in \mathrm{Ob}(\mathfrak{S}(U))$ and $\mathcal{F}^{U^{\prime}} \in \mathrm{Ob}\left(\mathfrak{S}\left(U^{\prime}\right)\right)$. There is a natural equivalence

$$
f_{*} \mathfrak{H o m}\left(f^{-1} \mathfrak{S}, \mathfrak{T}\right) \stackrel{\approx}{\rightarrow} \mathfrak{H o m}\left(\mathfrak{S}, f_{*} \mathfrak{T}\right) \text {. }
$$

\subsection{Linear stacks.}

As a matter of conventions, in this paper rings are unitary, and ring homomorphisms preserve the unit. If $R$ is a commutative ring, we call $R$-algebra a not necessarily commutative ring $A$ endowed with a ring homomorphism $R \rightarrow A$ whose image is in the center of $A$.

Let $R$ be a commutative ring. An $R$-linear category, that we will call $R$-category for short, is a category $C$ whose morphism sets are endowed with a structure of $R$-module such that composition is $R$-bilinear. An $R$-functor is a functor which is $R$-linear at the level of morphisms. Transformations of $R$-functors are simply transformations of the underlying functors. Note that if $\mathrm{D}$ is another $R$-category, the 
category $\mathrm{Hom}_{R}(\mathrm{C}, \mathrm{D})$ of $R$-functors and transformations is again an $R$-category, the $R$-module structure on the sets of transformations being defined object-wise.

For each $c \in \mathrm{Ob}(\mathrm{C})$ the set of endomorphisms $\operatorname{End}_{\mathrm{C}}(c)$ has a natural structure of $R$-algebra, with product given by composition. In particular, note that $R$ algebras are identified with $R$-categories with a single object. Let us denote for short by End (id $\mathrm{C}$ ) the $R$-algebra End $_{\mathrm{End}_{R}(\mathrm{C})}\left(\mathrm{id}_{\mathrm{C}}\right)$. It is a commutative ${ }^{5} R$-algebra, called the center of $C$. Note that $C$ is an $R$-category if and only if $C$ is a $\mathbb{Z}$ category (also called preadditive category) endowed with a ring homomorphism $R \rightarrow$ End (idc).

Definition 1.7. (a) An $R$-linear stack, that we will call $R$-stack for short, is a stack $\mathfrak{S}$ such that $\mathfrak{S}(U)$ is an $R$-category for every open subset $U$, and whose restrictions are $R$-functors. An $R$-functor of $R$-stacks is a functor which is linear at the level of morphisms. No additional requirements are imposed on transformations of $R$-functors.

(b) Let $\mathcal{R}$ be a sheaf of commutative rings on $X$. An $\mathcal{R}$-linear stack, that we will call $\mathcal{R}$-stack for short, is a $\mathbb{Z}$-stack $\mathfrak{S}$ whose center $\mathcal{E} n d\left(\mathrm{id}_{\mathfrak{S}}\right)$ is a sheaf of commutative $\mathcal{R}$-algebras ${ }^{6}$. There is a natural notion of $\mathcal{R}$-functor ${ }^{7}$, and transformations of $\mathcal{R}$-functors are just transformations of the underlying functors.

One says that an $\mathcal{R}$-functor $\varphi: \mathfrak{S} \rightarrow \mathfrak{T}$ is an equivalence (resp. admits a right or a left adjoint) if it is so forgetting the $\mathcal{R}$-linear structure. Note that a quasiinverse to $\varphi$ (resp. its right or left adjoint) is necessarily an $\mathcal{R}$-functor itself. One says that $\varphi: \mathfrak{S} \rightarrow \mathfrak{M o d}(\mathcal{R})$ is representable if there is an invertible transformation $\varphi \Rightarrow \mathcal{H o m}_{\mathfrak{S}}(\mathcal{F}, \cdot)$ for some $\mathcal{F} \in \mathfrak{S}(X)$.

\subsection{Operations.}

Let $\mathfrak{S}$ and $\mathfrak{S}^{\prime}$ be $\mathcal{R}$-stacks. The stack $\mathfrak{H o m}_{\mathcal{R}}\left(\mathfrak{S}, \mathfrak{S}^{\prime}\right)$ of $\mathcal{R}$-functors and transformations is an $\mathcal{R}$-stack. The product $\mathfrak{S} \otimes_{\mathcal{R}} \mathfrak{S}^{\prime}$ is the stack associated with the prestack

\footnotetext{
${ }^{5}$ Let $\alpha, \beta: \mathrm{id}_{\mathrm{C}} \Rightarrow \mathrm{id}_{\mathrm{C}}$ be transformations, and $c \in \mathrm{Ob}(\mathrm{C})$. By definition of transformation, applying $\alpha$ to the morphism $\beta(c)$ we get a commutative diagram

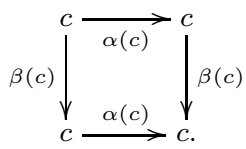

Note that the natural morphism End $\left(\operatorname{id}_{C}\right) \rightarrow \operatorname{End}_{C}(c), \alpha \mapsto \alpha(c)$, identifies End $\left(\operatorname{id}_{C}\right)$ canonically with the center of End $(c)$ for each $c \in \mathrm{Ob}(\mathrm{C})$

${ }^{6}$ By definition, this means that there is a morphism of sheaves of rings $\mu: \mathcal{R} \rightarrow \mathcal{E}$ nd $\left(\mathrm{id}_{\mathfrak{S}}\right)$. Note that the data of $\mu$ is equivalent to the requirement that for every open subset $U \subset X$, and any $\mathcal{F}, \mathcal{G} \in \mathfrak{S}(U)$ the sheaf $\mathcal{H o m}_{\left.\mathfrak{S}\right|_{U}}(\mathcal{F}, \mathcal{G})$ has a structure of $\left.\mathcal{R}\right|_{U}$-module compatible with restrictions, and such that composition is $\mathcal{R}$-bilinear.

${ }^{7}$ If $\mathfrak{S}^{\prime}=\left(\mathfrak{S}^{\prime}, \mu^{\prime}\right)$ is another $\mathcal{R}$-stack, an $\mathcal{R}$-functor $\varphi: \mathfrak{S} \rightarrow \mathfrak{S}^{\prime}$ is a functor of $\mathbb{Z}$-stacks such that $\varphi(\mu(r)(\mathcal{F}))=\mu^{\prime}(r)(\varphi(\mathcal{F}))$, as endomorphisms of $\varphi(\mathcal{F})$, for any $U \subset X, r \in \mathcal{R}(U)$, and $\mathcal{F} \in \mathrm{Ob}(\mathfrak{S}(U))$.
} 
$\mathfrak{S} \tilde{\otimes}_{\mathcal{R}} \mathfrak{S}^{\prime}$ defined as follows. At the level of objects, $\operatorname{Ob}\left(\mathfrak{S} \tilde{\otimes}_{\mathcal{R}} \mathfrak{S}^{\prime}\right)=\operatorname{Ob}(\mathfrak{S}) \times \mathrm{Ob}\left(\mathfrak{S}^{\prime}\right)$. At the level of morphisms,

$$
\operatorname{Hom}_{\mathfrak{S}_{\tilde{Q}_{\mathcal{R}} \mathfrak{S}^{\prime}(U)}}\left(\left(\mathcal{F}_{1}, \mathcal{F}_{1}^{\prime}\right),\left(\mathcal{F}_{2}, \mathcal{F}_{2}^{\prime}\right)\right)=\operatorname{Hom}_{\mathfrak{S}(U)}\left(\mathcal{F}_{1}, \mathcal{F}_{2}\right) \otimes_{\mathcal{R}} \operatorname{Hom}_{\mathfrak{S}^{\prime}(U)}\left(\mathcal{F}_{1}^{\prime}, \mathcal{F}_{2}^{\prime}\right) .
$$

If $\mathfrak{S}^{\prime \prime}$ is another $\mathcal{R}$-stack, there is a natural $\mathcal{R}$-equivalence

$$
\mathfrak{H o m}{ }_{\mathcal{R}}\left(\mathfrak{S} \otimes_{\mathcal{R}} \mathfrak{S}^{\prime}, \mathfrak{S}^{\prime \prime}\right) \stackrel{\approx}{\rightarrow} \mathfrak{H o m}_{\mathcal{R}}\left(\mathfrak{S}, \mathfrak{H o m} \mathfrak{m}_{\mathcal{R}}\left(\mathfrak{S}^{\prime}, \mathfrak{S}^{\prime \prime}\right)\right)
$$

Let $f: Y \rightarrow X$ be a continuous map of topological spaces, $\mathfrak{S}$ an $\mathcal{R}$-stack on $X$, and $\mathfrak{T}$ an $f^{-1} \mathcal{R}$-stack on $Y$. Then $f_{*} \mathfrak{T}$ is an $\mathcal{R}$-stack, $f^{-1} \mathfrak{S}$ is an $f^{-1} \mathcal{R}$-stack, and there is a natural equivalence

$$
f_{*} \mathfrak{H o m} f_{f^{-1} \mathcal{R}}\left(f^{-1} \mathfrak{S}, \mathfrak{T}\right) \stackrel{\approx}{\rightarrow} \mathfrak{H o m}_{\mathcal{R}}\left(\mathfrak{S}, f_{*} \mathfrak{T}\right) .
$$

\subsection{Stacks of twisted modules.}

Let $X$ be a topological space, $\mathcal{R}$ a sheaf of commutative rings on $X$, and $\mathcal{A}$ a sheaf of not necessarily commutative $\mathcal{R}$-algebras. Let $\operatorname{Mod}(\mathcal{A})$ be the category of $\mathcal{A}$-modules and $\mathcal{A}$-linear morphisms. Unless otherwise stated, by $\mathcal{A}$-module we mean here left $\mathcal{A}$-module. The prestack $\mathfrak{M} \mathfrak{o d}(\mathcal{A})$ of $\mathcal{A}$-modules on $X$ is defined by $U \mapsto \operatorname{Mod}\left(\left.\mathcal{A}\right|_{U}\right)$, with natural restriction functors. It is clearly an $\mathcal{R}$-stack.

Definition 1.8. (a) A stack of $\mathcal{R}$-twisted modules is an $\mathcal{R}$-stack which is locally $\mathcal{R}$-equivalent to stacks of modules over $\mathcal{R}$-algebras. More precisely, an $\mathcal{R}$ stack $\mathfrak{M}$ is a stack of $\mathcal{R}$-twisted modules if there exist an open covering $\left\{U_{i}\right\}_{i \in I}$ of $X,\left.\mathcal{R}\right|_{U_{i}}$-algebras $\mathcal{A}_{i}$ on $U_{i}$, and $\left.\mathcal{R}\right|_{U_{i}}$-equivalences of $\left.\mathcal{R}\right|_{U_{i}}$-stacks $\varphi_{i}:\left.\mathfrak{M}\right|_{U_{i}} \rightarrow \mathfrak{M o d}\left(\mathcal{A}_{i}\right)$.

(b) A stack of $\mathcal{R}$-twisted $\mathcal{A}$-modules is an $\mathcal{R}$-stack which is locally $\mathcal{R}$-equivalent to $\mathfrak{M} \mathfrak{o d}(\mathcal{A})$.

(c) A stack of twisted $\mathcal{R}$-modules is a stack of $\mathcal{R}$-twisted $\mathcal{R}$-modules.

If $\mathfrak{M}$ is a stack of $\mathcal{R}$-twisted modules (resp. a stack of $\mathcal{R}$-twisted $\mathcal{A}$-modules, resp. a stack of twisted $\mathcal{R}$-modules), objects of $\mathfrak{M}(X)$ are called $\mathcal{R}$-twisted modules (resp. $\mathcal{R}$-twisted $\mathcal{A}$-modules, resp. twisted $\mathcal{R}$-modules).

Recall that a stack $\mathfrak{M}$ is called additive if the categories $\mathfrak{M}(U)$ and the restriction functors are additive. A stack $\mathfrak{M}$ is called abelian if the categories $\mathfrak{M}(U)$ are abelian, and the restriction functors are exact. Since stacks of modules over $\mathcal{R}$-algebras are abelian, stacks of $\mathcal{R}$-twisted modules are also abelian.

Remark 1.9. The stacks constructed in [20, 28, 30] provide examples of stacks of twisted modules which are of an intermediate nature between (a) and (b) of Definition 1.8 With notations as in (a), denote by $\psi_{i}$ a quasi-inverse to $\varphi_{i}$. These are stacks of $\mathcal{R}$-twisted modules for which the equivalences $\left.\varphi_{i} \circ \psi_{j}\right|_{U_{i j}}$ are induced by isomorphisms of $\left.\mathcal{R}\right|_{U_{i j}}$-algebras $\left.\left.\mathcal{A}_{i}\right|_{U_{i j}} \stackrel{\sim}{\rightarrow} \mathcal{A}_{j}\right|_{U_{i j}}$. This is related to non-abelian cohomology as in [14, and we will discuss these matters in 9]. 
Recall that $\mathcal{A}$-modules are sheaves of $\mathcal{R}$-modules $\mathcal{F}$ endowed with a morphism of sheaves of rings $m: \mathcal{A} \rightarrow \mathcal{E} n d_{\mathcal{R}}(\mathcal{F})$.

Definition 1.10. If $\mathcal{A}$ is an $\mathcal{R}$-algebra and $\mathfrak{S}$ an $\mathcal{R}$-stack, we denote by $\mathfrak{M o d}(\mathcal{A} ; \mathfrak{S})$ the $\mathcal{R}$-stack whose objects on an open subset $U \subset X$ are pairs of an object $\mathcal{F} \in \mathfrak{S}(U)$ and a morphism of $\left.\mathcal{R}\right|_{U}$-algebras $m:\left.\mathcal{A}\right|_{U} \rightarrow \mathcal{E} n d_{\left.\mathfrak{S}\right|_{U}}(\mathcal{F})$, and whose morphisms are those morphisms in $\mathfrak{S}(U)$ commuting with $m$. We denote by $\operatorname{Mod}(\mathcal{A} ; \mathfrak{S})$ the category $\mathfrak{M o d}(\mathcal{A} ; \mathfrak{S})(X)$.

Let $\mathcal{A}$ and $\mathcal{B}$ be $\mathcal{R}$-algebras. Recall that an $\mathcal{A} \otimes_{\mathcal{R}} \mathcal{B}$-module is the same as a $\mathcal{B}$-module $\mathcal{M}$ endowed with an $\mathcal{R}$-algebra morphism $\mathcal{A} \rightarrow \mathcal{E} n d_{\mathcal{B}}(\mathcal{M})$. Hence, there is an $\mathcal{R}$-equivalence

$$
\mathfrak{M o d}(\mathcal{A} ; \mathfrak{M o d}(\mathcal{B})) \approx \mathfrak{M o d}\left(\mathcal{A} \otimes_{\mathcal{R}} \mathcal{B}\right) .
$$

In particular, if $\mathfrak{M}$ is a stack of $\mathcal{R}$-twisted modules (resp. of twisted $\mathcal{R}$-modules), then $\mathfrak{M o d}(\mathcal{A} ; \mathfrak{M})$ is a stack of $\mathcal{R}$-twisted modules (resp. of $\mathcal{R}$-twisted $\mathcal{A}$-modules).

\section{Operations}

Using Morita theory, we develop the formalism of operations for stacks of twisted modules. We then obtain Grothendieck's six operations for derived categories of twisted modules over locally compact Hausdorff topological spaces.

\subsection{Morita theory I. Functors admitting an adjoint.}

Morita theory describes in terms of bimodules functors between categories of modules which admit an adjoint (references are made to [1, 12]). We are interested in the local analogue of this result, dealing with stacks of modules over sheaves rings. Our reference was [25], where only the case of equivalences is discussed. We thus adapt here their arguments in order to deal with functors admitting an adjoint.

Let $\mathcal{R}$ be a sheaf of commutative rings on a topological space $X$, and let $\mathcal{A}$ be a sheaf of not necessarily commutative $\mathcal{R}$-algebras. Denote by $\mathcal{A}^{\text {op }}$ the opposite algebra to $\mathcal{A}$, given by $\mathcal{A}^{\mathrm{op}}=\left\{a^{\mathrm{op}}: a \in \mathcal{A}\right\}$ with product $a^{\mathrm{op}} b^{\mathrm{op}}=(b a)^{\mathrm{op}}$. Note that left (resp. right) $\mathcal{A}^{\mathrm{op}}$-modules are but right (resp. left) $\mathcal{A}$-modules.

For $\mathfrak{S}$ and $\mathfrak{S}^{\prime}$ two $\mathcal{R}$-stacks, denote by

$$
\mathfrak{H o m}_{\mathcal{R}}^{\mathrm{r}}\left(\mathfrak{S}, \mathfrak{S}^{\prime}\right)
$$

the full $\mathcal{R}$-substack of $\mathfrak{H o m}_{\mathcal{R}}\left(\mathfrak{S}, \mathfrak{S}^{\prime}\right)$ of functors that admit a right adjoint. This is equivalent to the opposite of the stack of $\mathcal{R}$-functors from $\mathfrak{S}^{\prime}$ to $\mathfrak{S}$ that admit a left adjoint. 
Proposition 2.1. Let $\mathcal{A}$ and $\mathcal{B}$ be $\mathcal{R}$-algebras. The functor

$$
\Phi: \mathfrak{M o d}\left(\mathcal{A} \otimes_{\mathcal{R}} \mathcal{B}^{\mathrm{op}}\right) \rightarrow \mathfrak{H o m}_{\mathcal{R}}^{\mathrm{r}}(\mathfrak{M o d}(\mathcal{B}), \mathfrak{M o d}(\mathcal{A}))
$$

given by $\mathcal{L} \mapsto \mathcal{L} \otimes_{\mathcal{B}}(\cdot)$ is an $\mathcal{R}$-equivalence.

It follows that $\mathcal{R}$-functors $\mathfrak{M o d}(\mathcal{A}) \rightarrow \mathfrak{M o d}(\mathcal{B})$ which admit a left adjoint are of the form $\mathcal{H o m}_{\mathcal{A}}(\mathcal{L}, \cdot)$, for an $\mathcal{A} \otimes_{\mathcal{R}} \mathcal{B}^{\text {op }}$-module $\mathcal{L}$.

Proof. (We follow here arguments similar to those in the proof of Morita theorem given in [25].) One checks that $\Phi$ is fully faithful. Let us show that it is essentially surjective. Let $\varphi: \mathfrak{M o d}(\mathcal{B}) \rightarrow \mathfrak{M o d}(\mathcal{A})$ be an $\mathcal{R}$-functor admitting a right adjoint. The $\mathcal{A}$-module $\mathcal{L}=\varphi(\mathcal{B})$ inherits a compatible $\mathcal{B}^{\text {op }}$-module structure by that of $\mathcal{B}$ itself, and we set $\varphi^{\prime}(\cdot)=\mathcal{L} \otimes_{\mathcal{B}}(\cdot)$. A transformation $\alpha: \varphi^{\prime} \Rightarrow \varphi$ is defined as follows. For $U \subset X$ and $\mathcal{N} \in \operatorname{Mod}\left(\left.\mathcal{B}\right|_{U}\right)$, the morphism

$$
\alpha(\mathcal{N}):\left.\varphi(\mathcal{B})\right|_{U} \otimes_{\left.\mathcal{B}\right|_{U}} \mathcal{N} \rightarrow \varphi(\mathcal{N})
$$

is given by $l \otimes n \mapsto \varphi(\widetilde{n})(l)$, where $\widetilde{n}:\left.\mathcal{B}\right|_{U} \rightarrow \mathcal{N}$ denotes the map $b \mapsto b n$. We have to prove that $\alpha(\mathcal{N})$ is an isomorphism. The $\left.\mathcal{B}\right|_{U}$-module $\mathcal{N}$ admits a presentation $\bigoplus_{j} \mathcal{B}_{U_{j}} \rightarrow \bigoplus_{i} \mathcal{B}_{U_{i}} \rightarrow \mathcal{N} \rightarrow 0$, where one sets $(\cdot)_{U}=u ! u^{-1}$ for $u: U \rightarrow X$ the open inclusion. We may then assume that $\mathcal{N}=\bigoplus_{i} \mathcal{B}_{U_{i}}$. Since $\varphi$ and $\varphi^{\prime}$ admit a right adjoint, one has $\varphi\left(\bigoplus_{i} \mathcal{B}_{U_{i}}\right) \simeq \bigoplus_{i} \varphi(\mathcal{B})_{U_{i}}$, and $\varphi^{\prime}\left(\bigoplus_{i} \mathcal{B}_{U_{i}}\right) \simeq \bigoplus_{i} \varphi^{\prime}(\mathcal{B})_{U_{i}}$ by Lemma 2.2. Hence we are reduced to prove the isomorphism $\varphi\left(\left.\mathcal{B}\right|_{U}\right) \otimes_{\left.\mathcal{B}\right|_{U}} \mathcal{B}_{U} \stackrel{\sim}{\rightarrow}$ $\varphi\left(\left.\mathcal{B}\right|_{U}\right)$, which is obvious.

Lemma 2.2. Let $\mathcal{A}$ and $\mathcal{B}$ be $\mathcal{R}$-algebras, and let $\varphi: \mathfrak{M o d}(\mathcal{B}) \rightarrow \mathfrak{M o d}(\mathcal{A})$ be an $\mathcal{R}$-functor admitting a right adjoint. Then for any family of open subsets $\left\{U_{i}\right\}_{i \in I}$ of $U \subset X$, and any $\mathcal{N} \in \operatorname{Mod}\left(\left.\mathcal{B}\right|_{U}\right)$, one has $\varphi\left(\bigoplus_{i} \mathcal{N}_{U_{i}}\right) \simeq \bigoplus_{i} \varphi(\mathcal{N})_{U_{i}}$.

Proof. The proof is straightforward. We leave it to the reader to check that a functor admitting a right adjoint commutes with inductive limits, and in particular with direct sums. Let us check that $\varphi\left(\mathcal{N}_{V}\right) \simeq \varphi(\mathcal{N})_{V}$ for an open inclusion $v: V \rightarrow$ $U$. Let $\psi$ be a right adjoint to $\varphi$. Note that the proper direct image $v_{!}$is left adjoint to the restriction functor $v^{-1}(\cdot)=\left.(\cdot)\right|_{V}$. For every $\mathcal{M} \in \operatorname{Mod}\left(\left.\mathcal{A}\right|_{U}\right)$ one has

$$
\begin{aligned}
\operatorname{Hom}_{\left.\mathcal{A}\right|_{U}}\left(\varphi\left(\mathcal{N}_{V}\right), \mathcal{M}\right) & =\operatorname{Hom}_{\left.\mathcal{A}\right|_{U}}\left(\varphi\left(v_{!}\left(\left.\mathcal{N}\right|_{V}\right)\right), \mathcal{M}\right) \\
& \simeq \operatorname{Hom}_{\left.\mathcal{B}\right|_{V}}\left(\left.\mathcal{N}\right|_{V},\left.\psi(\mathcal{M})\right|_{V}\right) \\
& \simeq \operatorname{Hom}_{\left.\mathcal{B}\right|_{V}}\left(\left.\mathcal{N}\right|_{V}, \psi\left(\left.\mathcal{M}\right|_{V}\right)\right) \\
& \simeq \operatorname{Hom}_{\left.\mathcal{A}\right|_{U}}\left(v_{!}\left(\varphi\left(\left.\mathcal{N}\right|_{V}\right)\right), \mathcal{M}\right) \\
& \simeq \operatorname{Hom}_{\left.\mathcal{A}\right|_{U}}\left(v_{!}\left(\left.\varphi(\mathcal{N})\right|_{V}\right), \mathcal{M}\right) \\
& =\operatorname{Hom}_{\left.\mathcal{A}\right|_{U}}\left(\varphi(\mathcal{N})_{V}, \mathcal{M}\right),
\end{aligned}
$$

where the second and fourth isomorphisms follow from the fact that $\psi$ and $\varphi$, respectively, are functors of stacks. 


\subsection{Internal product of stacks of twisted modules.}

We are now ready to define duality and internal product for stacks of twisted modules.

Let $\mathcal{R}$ be a sheaf of commutative rings on a topological space $X$. Recall that for $\mathfrak{S}$ and $\mathfrak{S}^{\prime}$ two $\mathcal{R}$-stacks, we denote by $\mathfrak{H o m}_{\mathcal{R}}^{\mathrm{r}}\left(\mathfrak{S}, \mathfrak{S}^{\prime}\right)$ the stack of $\mathcal{R}$-functors that admit a right adjoint.

Definition 2.3. Let $\mathfrak{S}$ and $\mathfrak{S}^{\prime}$ be $\mathcal{R}$-stacks on $X$. Set

$$
\begin{aligned}
\mathfrak{S}^{\circledast-1} & =\mathfrak{H o m}_{\mathcal{R}}^{\mathrm{r}}(\mathfrak{S}, \mathfrak{M o d}(\mathcal{R})), \\
\mathfrak{S} \circledast_{\mathcal{R}} \mathfrak{S}^{\prime} & =\mathfrak{H o m}_{\mathcal{R}}^{\mathrm{r}}\left(\mathfrak{S}^{\circledast-1}, \mathfrak{S}^{\prime}\right) .
\end{aligned}
$$

Remark 2.4. The definition of $\mathfrak{S}^{\circledast-1}$ does depend on the ring $\mathcal{R}$, but we do not keep track of this dependence in the notation to avoid more cumbersome notations like $\mathfrak{S}^{\circledast \mathcal{R}}-1$.

As a consequence of Proposition 2.1 and equivalence (1.5), we have

Proposition 2.5. If $\mathcal{A}$ and $\mathcal{A}^{\prime}$ are $\mathcal{R}$-algebras, there are $\mathcal{R}$-equivalences

$$
\begin{aligned}
\mathfrak{M o d}(\mathcal{A})^{\circledast-1} & \approx \operatorname{Mod}\left(\mathcal{A}^{\mathrm{op}}\right), \\
\mathfrak{M o d}(\mathcal{A}) \circledast_{\mathcal{R}} \mathfrak{M o d}\left(\mathcal{A}^{\prime}\right) & \approx \operatorname{Mod}\left(\mathcal{A} \otimes_{\mathcal{R}} \mathcal{A}^{\prime}\right) . \\
& \approx \operatorname{Mod}\left(\mathcal{A} ; \mathfrak{M o d}\left(\mathcal{A}^{\prime}\right)\right) .
\end{aligned}
$$

In particular, if $\mathfrak{M}$ and $\mathfrak{M}^{\prime}$ are stacks of $\mathcal{R}$-twisted modules on $X$, then $\mathfrak{M}^{\circledast-1}$ and $\mathfrak{M} \circledast_{\mathcal{R}} \mathfrak{M}^{\prime}$ are stacks of $\mathcal{R}$-twisted modules on $X$.

Let us list some properties of these operations.

Lemma 2.6. Let $\mathcal{A}$ be an $\mathcal{R}$-algebra, and $\mathfrak{S}$ and $\mathfrak{S}^{\prime}$ be $\mathcal{R}$-stacks. Then there are natural $\mathcal{R}$-functors

$$
\begin{aligned}
\mathfrak{M o d}(\mathcal{A}) \circledast_{\mathcal{R}} \mathfrak{S} & \rightarrow \mathfrak{M o d}(\mathcal{A} ; \mathfrak{S}), \\
\mathfrak{S} & \rightarrow\left(\mathfrak{S}^{\circledast-1}\right)^{\circledast-1}=\mathfrak{S}_{\mathcal{R}} \mathfrak{M} \mathfrak{M o d}(\mathcal{R}), \\
\mathfrak{H o m}_{\mathcal{R}}^{\mathrm{r}}\left(\mathfrak{S}, \mathfrak{S}^{\prime}\right) & \rightarrow \mathfrak{H o m}_{\mathcal{R}}^{\mathrm{r}}\left(\mathfrak{S}^{\circledast-1}, \mathfrak{S}^{\circledast-1}\right)=\mathfrak{S}^{\prime} \circledast_{\mathcal{R}} \mathfrak{S}^{\circledast-1} .
\end{aligned}
$$

Proof. In the identification $\mathfrak{M o d}(\mathcal{A}) \circledast_{\mathcal{R}} \mathfrak{S} \approx \mathfrak{H o m}_{\mathcal{R}}^{\mathrm{r}}\left(\mathfrak{M} \mathfrak{o d}\left(\mathcal{A}^{\mathrm{op}}\right), \mathfrak{S}\right)$, the functor (2.3) is given by $\phi \mapsto(\mathcal{F}, m)$, where $\mathcal{F}=\phi\left(\mathcal{A}^{\mathrm{op}}\right)$, and $m: \mathcal{A} \simeq \mathcal{E} n d_{\mathcal{A}^{\mathrm{op}}}\left(\mathcal{A}^{\mathrm{op}}\right) \stackrel{\phi}{\rightarrow}$ $\mathcal{E}_{n d}\left(\phi\left(\mathcal{A}^{\mathrm{op}}\right)\right)$.

The functor 2.4) is given by $\mathcal{F} \mapsto(\phi \mapsto \phi(\mathcal{F}))$, using the identification $\left(\mathfrak{S}^{\circledast-1}\right)^{\circledast-1}=\mathfrak{H o m}_{\mathcal{R}}^{\mathrm{r}}\left(\mathfrak{H}_{\mathfrak{O}} \mathfrak{m}_{\mathcal{R}}^{\mathrm{r}}(\mathfrak{S}, \mathfrak{M o d}(\mathcal{R})), \mathfrak{M o d}(\mathcal{R})\right)$.

Finally, the functor (2.5) is given by $\varphi \mapsto(\psi \mapsto \psi \circ \varphi)$, using the identification $\mathfrak{H o m}_{\mathcal{R}}^{\mathrm{r}}\left(\mathfrak{S}^{\prime \circledast-1}, \mathfrak{S}^{\circledast-1}\right)=\mathfrak{H o m}_{\mathcal{R}}^{\mathrm{r}}\left(\mathfrak{H o m}_{\mathcal{R}}^{\mathrm{r}}\left(\mathfrak{S}^{\prime}, \mathfrak{M o d}(\mathcal{R})\right), \mathfrak{H o m}_{\mathcal{R}}^{\mathrm{r}}(\mathfrak{S}, \mathfrak{M o d}(\mathcal{R}))\right)$.

We need the following lemma from [25]. 
Lemma 2.7. For $\mathfrak{M}$ a stack of $\mathcal{R}$-twisted modules, there is a natural $\mathcal{R}$-functor

$$
\otimes_{\mathcal{R}}: \mathfrak{M o d}(\mathcal{R}) \times \mathfrak{M} \rightarrow \mathfrak{M}
$$

Proof. For $\mathcal{M} \in \operatorname{Mod}(\mathcal{R})$ and $\mathcal{F} \in \mathfrak{M}(X)$, the functor

$$
\mathcal{H o m}_{\mathcal{R}}\left(\mathcal{M}, \mathcal{H o m} m_{\mathfrak{M}}(\mathcal{F}, \cdot)\right): \mathfrak{M} \rightarrow \mathfrak{M o d}(\mathcal{R})
$$

is locally (and hence globally) representable, and we denote by $\mathcal{M} \otimes_{\mathcal{R}} \mathcal{F}$ a representative.

Proposition 2.8. Let $\mathfrak{M}, \mathfrak{M}^{\prime}$, and $\mathfrak{M}^{\prime \prime}$ be stacks of $\mathcal{R}$-twisted modules. Then there is a natural $\mathcal{R}$-equivalence

$$
\mathfrak{H o m}{ }_{\mathcal{R}}^{\mathrm{r}}\left(\mathfrak{M} \circledast_{\mathcal{R}} \mathfrak{M}^{\prime}, \mathfrak{M}^{\prime \prime}\right) \stackrel{\approx}{\rightarrow} \mathfrak{H o m}_{\mathcal{R}}^{\mathrm{r}}\left(\mathfrak{M}, \mathfrak{H} \mathfrak{o m} \mathfrak{m}_{\mathcal{R}}^{\mathrm{r}}\left(\mathfrak{M}^{\prime}, \mathfrak{M}^{\prime \prime}\right)\right) .
$$

Proof. The above $\mathcal{R}$-functor is given by $\varphi \mapsto\left(\mathcal{F} \mapsto\left(\mathcal{F}^{\prime} \mapsto \varphi\left(\psi_{\mathcal{F}, \mathcal{F}^{\prime}}\right)\right)\right)$, where $\psi_{\mathcal{F}, \mathcal{F}^{\prime}} \in \mathfrak{M} \circledast_{\mathcal{R}} \mathfrak{M}^{\prime}=\mathfrak{H o m}_{\mathcal{R}}^{\mathrm{r}}\left(\mathfrak{H o m}_{\mathcal{R}}^{\mathrm{r}}(\mathfrak{M}, \mathfrak{M} \mathfrak{o d}(\mathcal{R})), \mathfrak{M}^{\prime}\right)$ is defined by $\psi_{\mathcal{F}, \mathcal{F}^{\prime}}(\eta)=$ $\eta(\mathcal{F}) \otimes_{\mathcal{R}} \mathcal{F}^{\prime}$. Here we used the $\mathcal{R}$-functor $\otimes_{\mathcal{R}}$ described in Lemma [2.7] We are then left to prove that this functor is a local equivalence. We may then assume that $\mathfrak{M} \approx \mathfrak{M}_{\mathfrak{o d}}(\mathcal{A}), \mathfrak{M}^{\prime} \approx \mathfrak{M o d}\left(\mathcal{A}^{\prime}\right)$, and $\mathfrak{M}^{\prime \prime} \approx \mathfrak{M} \mathfrak{o d}\left(\mathcal{A}^{\prime \prime}\right)$ for some $\mathcal{R}$-algebras $\mathcal{A}$, $\mathcal{A}^{\prime}$, and $\mathcal{A}^{\prime \prime}$. In this case both terms are equivalent to $\mathfrak{M o d}\left(\mathcal{A}^{\mathrm{op}} \otimes_{\mathcal{R}} \mathcal{A}^{\prime \mathrm{op}} \otimes_{\mathcal{R}} \mathcal{A}^{\prime \prime}\right)$.

Proposition 2.9. Let $\mathcal{A}$ be an $\mathcal{R}$-algebra, and let $\mathfrak{M}, \mathfrak{M}^{\prime}$, and $\mathfrak{M}^{\prime \prime}$ be stacks of $\mathcal{R}$-twisted modules. Then there are natural $\mathcal{R}$-equivalences

$$
\begin{aligned}
\mathfrak{M o d}(\mathcal{A}) \circledast_{\mathcal{R}} \mathfrak{M} & \approx \mathfrak{M o d}(\mathcal{A} ; \mathfrak{M}), \\
\mathfrak{M} & \approx\left(\mathfrak{M}^{\circledast-1}\right)^{\circledast-1}=\mathfrak{M}_{\mathcal{R}} \mathfrak{M o d}(\mathcal{R}), \\
\mathfrak{M}_{\mathcal{R}} \mathfrak{M}^{\prime} & \approx \mathfrak{M}^{\prime} \circledast_{\mathcal{R}} \mathfrak{M}, \\
\left(\mathfrak{M} \circledast_{\mathcal{R}} \mathfrak{M}^{\prime}\right)^{\circledast-1} & \approx \mathfrak{M}^{\circledast-1} \circledast_{\mathcal{R}} \mathfrak{M}^{\prime \circledast-1} \\
\left(\mathfrak{M} \circledast_{\mathcal{R}} \mathfrak{M}^{\prime}\right) \circledast_{\mathcal{R}} \mathfrak{M}^{\prime \prime} & \approx \mathfrak{M}_{\mathcal{R}}\left(\mathfrak{M}^{\prime} \circledast_{\mathcal{R}} \mathfrak{M}^{\prime \prime}\right) .
\end{aligned}
$$

Proof. Equivalences (2.6) and (2.7) follow by noticing that the functors (2.3) and (2.4) are local equivalences for $\mathfrak{S}=\mathfrak{M}$. The equivalence (2.8) follows by noticing that the functor (2.5) is locally an equivalence for $\mathfrak{S}=\mathfrak{M}^{\circledast-1}$ and $\mathfrak{S}^{\prime}=\mathfrak{M}^{\prime}$. The equivalence (2.9) follows from the chain of equivalences

$$
\begin{aligned}
& \mathfrak{H o m} \mathfrak{R}_{\mathcal{R}}^{\mathrm{r}}\left(\mathfrak{M} \circledast_{\mathcal{R}} \mathfrak{M}^{\prime}, \mathfrak{M o d}(\mathcal{R})\right) \approx \mathfrak{H o m} \mathfrak{m}_{\mathcal{R}}^{\mathrm{r}}\left(\mathfrak{M}, \mathfrak{H} \mathfrak{m}_{\mathcal{R}}^{\mathrm{r}}\left(\mathfrak{M}^{\prime}, \mathfrak{M} \mathfrak{o d}(\mathcal{R})\right)\right) \\
& \approx \mathfrak{H o m}_{\mathcal{R}}^{\mathrm{r}}\left(\left(\mathfrak{M}^{\circledast-1}\right)^{\circledast-1}, \mathfrak{M}^{\circledast \circledast-1}\right) .
\end{aligned}
$$

The equivalence (2.10) follows from the chain of equivalences

$$
\begin{aligned}
\mathfrak{H o m}_{\mathcal{R}}^{\mathrm{r}}\left(\left(\mathfrak{M} \circledast_{\mathcal{R}} \mathfrak{M}^{\prime}\right)^{\circledast-1}, \mathfrak{M}^{\prime \prime}\right) & \approx \mathfrak{H o m}_{\mathcal{R}}^{\mathrm{r}}\left(\mathfrak{M}^{\circledast-1} \circledast_{\mathcal{R}} \mathfrak{M}^{\circledast \circledast-1}, \mathfrak{M}^{\prime \prime}\right) \\
& \approx \mathfrak{H o m}_{\mathcal{R}}^{\mathrm{r}}\left(\mathfrak{M}^{\circledast-1}, \mathfrak{H o m}_{\mathcal{R}}^{\mathrm{r}}\left(\mathfrak{M}^{\prime \circledast-1}, \mathfrak{M}^{\prime \prime}\right)\right) .
\end{aligned}
$$

Let us describe a couple of other functors. There is a natural $\mathcal{R}$-functor

$$
\mathfrak{M o d}(\mathcal{R}) \rightarrow \mathfrak{M} \circledast_{\mathcal{R}} \mathfrak{M}^{\circledast-1},
$$


given by $\mathcal{F} \mapsto \mathcal{F} \otimes_{\mathcal{R}}(\cdot)$, in the identification $\mathfrak{M} \circledast{ }_{\mathcal{R}} \mathfrak{M}^{\circledast-1}=\mathfrak{H o m}_{\mathcal{R}}^{\mathrm{r}}\left(\mathfrak{M}^{\circledast-1}, \mathfrak{M}^{\circledast-1}\right)$. Locally, $\mathfrak{M} \approx \mathfrak{M o d}(\mathcal{A})$ for some $\mathcal{R}$-algebra $\mathcal{A}$, and the above functor coincides with $\mathfrak{M o d}(\mathcal{R}) \rightarrow \mathfrak{M o d}\left(\mathcal{A} \otimes_{\mathcal{R}} \mathcal{A}^{\mathrm{op}}\right), \mathcal{F} \mapsto \mathcal{F} \otimes_{\mathcal{R}} \mathcal{A}$. This has a right adjoint $\mathfrak{M o d}\left(\mathcal{A} \otimes_{\mathcal{R}}\right.$ $\left.\mathcal{A}^{\mathrm{op}}\right) \rightarrow \mathfrak{M o d}(\mathcal{R}), \mathcal{M} \mapsto Z(\mathcal{M})$, where $Z(\mathcal{M})=\mathcal{H} m_{\mathcal{A} \otimes_{\mathcal{R}} \mathcal{A}^{\text {op }}}(\mathcal{A}, \mathcal{M})=\{m \in$ $\mathcal{M}: a m=m a, \forall a \in \mathcal{A}\}$. Hence there is a right adjoint to (2.11)

$$
\mathfrak{M} \circledast_{\mathcal{R}} \mathfrak{M}^{\circledast-1} \rightarrow \mathfrak{M o d}(\mathcal{R}) .
$$

Note also that the forgetful functor

$$
\mathfrak{M o d}(\mathcal{A} ; \mathfrak{M}) \rightarrow \mathfrak{M}
$$

has (locally, and hence globally) a right adjoint

$$
\mathcal{A} \otimes_{\mathcal{R}}(\cdot): \mathfrak{M} \rightarrow \mathfrak{M o d}(\mathcal{A} ; \mathfrak{M})
$$

\subsection{Morita theory II. Relative case.}

In order to describe the pull-back functor for stacks of twisted modules, we need the following relative versions of the results in Section 2.1

Let $f: Y \rightarrow X$ be a continuous map of topological spaces, $\mathcal{R}$ a sheaf of commutative rings on $X, \mathfrak{S}$ an $\mathcal{R}$-stack, and $\mathfrak{T}$ an $f^{-1} \mathcal{R}$-stack. Denote by

$$
\mathfrak{H} \mathfrak{m}_{f^{-1} \mathcal{R}}^{\mathrm{r}-f_{*}}\left(f^{-1} \mathfrak{S}, \mathfrak{T}\right)
$$

the full $f^{-1} \mathcal{R}$-substack of $\mathfrak{H o m}_{f^{-1} \mathcal{R}}\left(f^{-1} \mathfrak{S}, \mathfrak{T}\right)$ of functors $\psi$ whose image by (1.4) belongs to $\mathfrak{H o m}_{\mathcal{R}}^{\mathrm{r}}\left(\mathfrak{S}, f_{*} \mathfrak{T}\right)$.

Proposition 2.10. Let $f: Y \rightarrow X$ be a continuous map of topological spaces, $\mathcal{B}$ an $\mathcal{R}$-algebra on $X$, and $\mathcal{C}$ an $f^{-1} \mathcal{R}$-algebra on $Y$. The functor

$$
\Psi: \mathfrak{M o d}\left(\mathcal{C} \otimes_{f^{-1} \mathcal{R}} f^{-1} \mathcal{B}^{\mathrm{op}}\right) \rightarrow \mathfrak{H o m}_{\mathcal{R}}^{\mathrm{r}-f_{*}}\left(f^{-1} \mathfrak{M o d}(\mathcal{B}), \mathfrak{M o d}(\mathcal{C})\right)
$$

given by $\mathcal{F} \mapsto \mathcal{F} \otimes_{f^{-1} \mathcal{B}}(\cdot)$ is an $f^{-1} \mathcal{R}$-equivalence.

Proof. The proof of this proposition is similar to that of Proposition 2.1 and we only show the essential surjectivity of $\Psi$. Let $\psi: f^{-1} \mathfrak{M o d}(\mathcal{B}) \rightarrow \mathfrak{M o d}(\mathcal{C})$ be an $f^{-1} \mathcal{R}$-functor such that $f_{*} \psi$ admits a right adjoint. Set $\mathcal{F}=\psi\left(f^{-1} \mathcal{B}\right)$ and $\psi^{\prime}(\cdot)=\mathcal{F} \otimes_{f^{-1} \mathcal{B}}(\cdot)$. For $V \subset Y$ and $\mathcal{N} \in f^{-1} \mathfrak{M o d}(\mathcal{B})(V)$, we have to check that the morphism

$$
\beta(\mathcal{N}):\left.\psi\left(\left.f^{-1} \mathcal{B}\right|_{V}\right) \otimes_{f-1} \mathcal{B}\right|_{V} \mathcal{N} \rightarrow \psi(\mathcal{N})
$$

defined as the morphism $\alpha$ in Proposition 2.1 is an isomorphism. By the definition of pull-back for stacks, $\mathcal{N}$ locally admits a presentation $\bigoplus_{k}^{\prime} f^{-1} \mathcal{M}_{k} \rightarrow \mathcal{N}$, where $\mathcal{M}_{k}$ are objects of $\mathfrak{M o d}(\mathcal{B})$ and $\bigoplus_{k}^{\prime}$ means that the sum is finite. Thus any $y \in Y$ has an open neighborhood $W \subset V$ such that there is a presentation

$$
\left.\left.\left.\bigoplus_{k}^{\prime} f^{-1}\left(\bigoplus_{j} \mathcal{B}_{U_{j k}}\right)\right|_{W} \rightarrow \bigoplus_{k}^{\prime} f^{-1}\left(\bigoplus_{i} \mathcal{B}_{U_{i k}}\right)\right|_{W} \rightarrow \mathcal{N}\right|_{W} \rightarrow 0
$$


Since $f_{*} \psi$ admits a right adjoint, one has

$$
\psi\left(\left.\bigoplus_{k}^{\prime} f^{-1}\left(\bigoplus_{i} \mathcal{B}_{U_{i k}}\right)\right|_{W}\right)=\bigoplus_{k}^{\prime} \bigoplus_{i} \psi\left(\left.f^{-1} \mathcal{B}\right|_{W}\right)_{f^{-1}\left(U_{i k}\right) \cap W}
$$

A similar formula holds for $\psi^{\prime}$, since also $f_{*} \psi^{\prime}$ admits a right adjoint. Hence we are reduced to prove the isomorphism $\left.\psi\left(\left.f^{-1} \mathcal{B}\right|_{W}\right) \otimes_{\left.f^{-1} \mathcal{B}\right|_{W}} f^{-1} \mathcal{B}\right|_{W} \stackrel{\sim}{\rightarrow} \psi\left(\left.f^{-1} \mathcal{B}\right|_{W}\right)$, which is obvious.

\subsection{Pull-back of stacks of twisted modules.}

We can now define the pull back of stacks of twisted modules.

Let $f: Y \rightarrow X$ be a continuous map of topological spaces, $\mathcal{R}$ a sheaf of commutative rings on $X, \mathfrak{S}$ an $\mathcal{R}$-stack, and $\mathfrak{T}$ an $f^{-1} \mathcal{R}$-stack. Recall that we denote by $\mathfrak{H o m}_{f^{-1} \mathcal{R}}^{\mathrm{r} f_{*}}\left(f^{-1} \mathfrak{S}, \mathfrak{T}\right)$ the $f^{-1} \mathcal{R}$-stack of functors $\psi$ whose image by (1.4) admits a right adjoint.

Definition 2.11. With the above notations, set

$$
f^{\circledast} \mathfrak{S}=\mathfrak{H o m}_{f^{-1} \mathcal{R}}^{\mathrm{r}-f_{*}}\left(f^{-1}\left(\mathfrak{S}^{\circledast-1}\right), \mathfrak{M o d}\left(f^{-1} \mathcal{R}\right)\right) .
$$

Remark 2.12. Again, as in Remark 2.4 we prefer the notation $f^{\circledast} \mathfrak{S}$ to the more cumbersome $f^{\circledast \mathcal{R}} \mathfrak{S}$.

As a consequence of Proposition 2.10 we have

Proposition 2.13. Let $f: Y \rightarrow X$ be a continuous map of topological spaces, and $\mathcal{A}$ an $\mathcal{R}$-algebra on $X$. Then, there is an $f^{-1} \mathcal{R}$-equivalence

$$
f^{\circledast} \mathfrak{M o d}(\mathcal{A}) \approx \mathfrak{M o d}\left(f^{-1} \mathcal{A}\right) .
$$

In particular, if $\mathfrak{M}$ a stack of $\mathcal{R}$-twisted modules, then $f^{\circledast \mathfrak{M}}$ is a stack of $f^{-1} \mathcal{R}$ twisted modules.

Let us list some properties of this operation.

Proposition 2.14. If $\mathfrak{S}$ is an $\mathcal{R}$-stack, there is a natural $\mathcal{R}$-functor

$$
f^{-1}: \mathfrak{S} \rightarrow f_{*} f^{\circledast} \mathfrak{S} .
$$

Proof. The usual sheaf-theoretical pull-back operation gives an $\mathcal{R}$-functor

$$
f^{-1}: \mathfrak{M o d}(\mathcal{R}) \rightarrow f_{*} f^{\circledast} \mathfrak{M o d}(\mathcal{R}) \approx f_{*} \mathfrak{M o d}\left(f^{-1} \mathcal{R}\right) .
$$


The functor in the statement is then obtained as the composition

$$
\begin{aligned}
\mathfrak{S} & \stackrel{\text { 2.4 }}{\longrightarrow} \mathfrak{S} \circledast_{\mathcal{R}} \mathfrak{M o d}(\mathcal{R}) \\
& \stackrel{\operatorname{id}_{\mathfrak{S}} \circledast_{\mathcal{R}} f^{-1}}{\longrightarrow} \mathfrak{S} \circledast_{\mathcal{R}} f_{*} \mathfrak{M o d}\left(f^{-1} \mathcal{R}\right) \\
& \approx \mathfrak{H o m}_{\mathcal{R}}^{\mathrm{r}}\left(\mathfrak{S}^{\circledast-1}, f_{*} \mathfrak{M o d}\left(f^{-1} \mathcal{R}\right)\right) \\
& \approx f_{*} \mathfrak{H o m}_{f^{-1} \mathcal{R}}^{\mathrm{r} f_{*}}\left(f^{-1}\left(\mathfrak{S}^{\circledast-1}\right), \mathfrak{M o d}\left(f^{-1} \mathcal{R}\right)\right) \\
& \approx f_{*} f^{\circledast} \mathfrak{S} .
\end{aligned}
$$

Proposition 2.15. Let $\mathfrak{M}$ be a stack of $\mathcal{R}$-twisted modules, and $\mathfrak{N}$ a stack of $f^{-1} \mathcal{R}$-twisted modules. Then there is a natural $\mathcal{R}$-equivalence

$$
f_{*} \mathfrak{H}_{\mathfrak{o m}}{ }_{f^{-1} \mathcal{R}}^{\mathrm{r}}\left(f^{\circledast} \mathfrak{M}, \mathfrak{N}\right) \stackrel{\approx}{\rightarrow} \mathfrak{H o m}_{\mathcal{R}}^{\mathrm{r}}\left(\mathfrak{M}, f_{*} \mathfrak{N}\right) .
$$

Proof. The functor $f^{-1}: \mathfrak{M} \rightarrow f_{*} f^{\circledast} \mathfrak{M}$ of Proposition 2.14 is locally the usual sheaf-theoretical pull-back, which has a right adjoint in the sheaf-theoretical pushforward. Moreover, it induces by (1.4) an $f^{-1} \mathcal{R}$-functor

$$
f^{-1} \mathfrak{M} \rightarrow f^{\circledast} \mathfrak{M} \text {. }
$$

Hence we get a functor

$$
\mathfrak{H} \mathfrak{o m}{ }_{f^{-1} \mathcal{R}}^{\mathrm{r}}\left(f^{\circledast} \mathfrak{M}, \mathfrak{N}\right) \rightarrow \mathfrak{H} \mathfrak{m}_{f^{-1} \mathcal{R}}^{\mathrm{r}-f_{*}}\left(f^{-1} \mathfrak{M}, \mathfrak{N}\right) .
$$

This is a local (and hence global) equivalence. We thus have the chain of equivalences

$$
\begin{aligned}
f_{*} \mathfrak{H o m}_{f^{-1} \mathcal{R}}^{\mathrm{r}}\left(f^{\circledast} \mathfrak{M}, \mathfrak{N}\right) & \approx f_{*} \mathfrak{H} \mathfrak{o m}_{f^{-1} \mathcal{R}}^{\mathrm{r}-f_{*}}\left(f^{-1} \mathfrak{M}, \mathfrak{N}\right) \\
& \approx \mathfrak{H} \mathfrak{o} \mathfrak{m}_{\mathcal{R}}^{\mathrm{r}}\left(\mathfrak{M}, f_{*} \mathfrak{N}\right) .
\end{aligned}
$$

Proposition 2.16. Let $\mathfrak{M}$ and $\mathfrak{M}^{\prime}$ be stacks of $\mathcal{R}$-twisted modules. Then there are natural $f^{-1} \mathcal{R}$-equivalences

$$
\begin{aligned}
f^{\circledast}\left(\mathfrak{M}^{\circledast-1}\right) & \approx\left(f^{\circledast} \mathfrak{M}\right)^{\circledast-1}, \\
f^{\circledast}\left(\mathfrak{M} \circledast \Re_{\mathcal{R}} \mathfrak{M}^{\prime}\right) & \approx f^{\circledast \mathfrak{M} \circledast f^{-1} \mathcal{R}} f^{\circledast} \mathfrak{M}^{\prime} .
\end{aligned}
$$

Proof. The equivalence (2.14) follows from the chain of equivalences

$$
\begin{aligned}
\mathfrak{H o m}_{f^{-1} \mathcal{R}}^{\mathrm{r}-f_{*}}\left(f^{-1}\left(\left(\mathfrak{M}^{\circledast-1}\right)^{\circledast-1}\right), \mathfrak{M o d}\left(f^{-1} \mathcal{R}\right)\right) & \approx \mathfrak{H o m}_{f^{-1} \mathcal{R}}^{\mathrm{r}-f_{*}}\left(f^{-1} \mathfrak{M}, \mathfrak{M o d}\left(f^{-1} \mathcal{R}\right)\right) \\
& \approx \mathfrak{H o m}_{f^{-1} \mathcal{R}}\left(f^{\circledast} \mathfrak{M}, \mathfrak{M o d}\left(f^{-1} \mathcal{R}\right)\right) .
\end{aligned}
$$

To prove (2.15), note that, by functoriality of $f^{\circledast}$, to any $\mathcal{R}$-stacks $\mathfrak{S}$ and $\mathfrak{S}^{\prime}$ is associated an $\mathcal{R}$-functor

$$
f^{\circledast}: \mathfrak{H o m}_{\mathcal{R}}^{\mathrm{r}}\left(\mathfrak{S}, \mathfrak{S}^{\prime}\right) \rightarrow f_{*} \mathfrak{H o m}_{f^{-1} \mathcal{R}}^{\mathrm{r}}\left(f^{\circledast} \mathfrak{S}, f^{\circledast} \mathfrak{S}^{\prime}\right)
$$


For $\mathfrak{S}=\mathfrak{M}^{\circledast-1}$ and $\mathfrak{S}^{\prime}=\mathfrak{M}^{\prime}$ this is locally the sheaf-theoretical pull-back functor

$$
f^{-1}: \mathfrak{M o d}\left(\mathcal{A} \otimes_{\mathcal{R}} \mathcal{A}^{\prime}\right) \rightarrow f_{*} \mathfrak{M o d}\left(f^{-1}\left(\mathcal{A} \otimes_{\mathcal{R}} \mathcal{A}^{\prime}\right)\right),
$$

which has a right adjoint. Hence $f^{\circledast}$ has a right adjoint, i.e.

$$
f^{\circledast} \in \mathfrak{H o m}_{\mathcal{R}}^{\mathrm{r}}\left(\mathfrak{H}_{\mathfrak{L}} \mathfrak{m}_{\mathcal{R}}^{\mathrm{r}}\left(\mathfrak{M}^{\circledast-1}, \mathfrak{M}^{\prime}\right), f_{*} \mathfrak{H} \mathfrak{m}_{f^{-1} \mathcal{R}}^{\mathrm{r}}\left(f^{\circledast}\left(\mathfrak{M}^{\circledast-1}\right), f^{\circledast} \mathfrak{M}^{\prime}\right)\right) .
$$

By Proposition 2.15 we get a functor

$$
f^{\circledast} \mathfrak{H o m}_{\mathcal{R}}^{\mathrm{r}}\left(\mathfrak{M}^{\circledast-1}, \mathfrak{M}^{\prime}\right) \rightarrow \mathfrak{H o m}_{f^{-1} \mathcal{R}}^{\mathrm{r}}\left(f^{\circledast}\left(\mathfrak{M}^{\circledast-1}\right), f^{\circledast} \mathfrak{M}^{\prime}\right) .
$$

This is locally, and hence globally, an equivalence.

\subsection{Twisted sheaf-theoretical operations.}

Let us now show how the usual operations of sheaf theory extend to the twisted case. For the classical non-twisted case, that we do not recall here, we refer e.g. to [22].

Proposition 2.17. Let $f: Y \rightarrow X$ be a continuous map of topological spaces, and $\mathfrak{M}$ and $\mathfrak{M}^{\prime}$ be stacks of $\mathcal{R}$-twisted modules. Then there exist $\mathcal{R}$-functors

$$
\begin{aligned}
\otimes_{\mathcal{R}} & : \mathfrak{M} \times \mathfrak{M}^{\prime} \rightarrow \mathfrak{M} \circledast_{\mathcal{R}} \mathfrak{M}^{\prime}, \\
\mathcal{H o m}_{\mathcal{R}} & :\left(\mathfrak{M}^{\circledast-1}\right)^{\mathrm{op}} \times \mathfrak{M}^{\prime} \rightarrow \mathfrak{M} \circledast_{\mathcal{R}} \mathfrak{M}^{\prime}, \\
f^{-1} & : \mathfrak{M} \rightarrow f_{*} f^{\circledast} \mathfrak{M}, \\
f_{*} & : f_{*} f^{\circledast} \mathfrak{M} \rightarrow \mathfrak{M} .
\end{aligned}
$$

If moreover $X$ and $Y$ are locally compact Hausdorff topological spaces, there exists an $\mathcal{R}$-functor

$$
f_{!}: f_{*} f^{\circledast} \mathfrak{M} \rightarrow \mathfrak{M} .
$$

If $U \subset X$ is an open subset where $\left.\mathfrak{M}\right|_{U} \approx \mathfrak{M o d}(\mathcal{A})$ and $\left.\mathfrak{M}^{\prime}\right|_{U} \approx \mathfrak{M o d}\left(\mathcal{A}^{\prime}\right)$ for some $\left.\mathcal{R}\right|_{U}$-algebras $\mathcal{A}$ and $\mathcal{A}^{\prime}$, then the restrictions to $U$ of the above functors coincide with the usual sheaf operations.

Proof. The functor

$$
\otimes_{\mathcal{R}}: \mathfrak{M} \times \mathfrak{M}^{\prime} \rightarrow \mathfrak{M} \circledast_{\mathcal{R}} \mathfrak{M}^{\prime}=\mathfrak{H o m}_{\mathcal{R}}^{\mathrm{r}}\left(\mathfrak{H o m}{ }_{\mathcal{R}}^{\mathrm{r}}(\mathfrak{M}, \mathfrak{M o d}(\mathcal{R})), \mathfrak{M}^{\prime}\right)
$$

is defined by $\left(\mathcal{F}, \mathcal{F}^{\prime}\right) \mapsto\left(\phi \mapsto \phi(\mathcal{F}) \otimes_{\mathcal{R}} \mathcal{F}^{\prime}\right)$, using Lemma 2.7

For $\mathcal{F}$ an object of $\mathfrak{M}^{\circledast-1}$ there is a natural functor

$$
\mathfrak{M} \circledast_{\mathcal{R}} \mathfrak{M}^{\prime} \rightarrow \mathfrak{M}^{\prime}
$$

given by $\phi \mapsto \phi(\mathcal{F})$ in the identification $\mathfrak{M}_{\mathcal{R}_{\mathcal{R}}} \mathfrak{M}^{\prime}=\mathfrak{H o m}_{\mathcal{R}}^{\mathrm{r}}\left(\mathfrak{M}^{\circledast-1}, \mathfrak{M}^{\prime}\right)$. Locally this corresponds to the functor $\mathfrak{M o d}\left(\mathcal{A} \otimes_{\mathcal{R}} \mathcal{A}^{\prime}\right) \rightarrow \mathfrak{M o d}\left(\mathcal{A}^{\prime}\right), \mathcal{M} \mapsto \mathcal{F} \otimes_{\mathcal{A}} \mathcal{M}$, for $\mathcal{F} \in \operatorname{Mod}\left(\mathcal{A}^{\text {op }}\right)$. If $\mathcal{N}$ is an $\mathcal{A}^{\prime}$-module, there is a functorial isomorphism $\mathcal{H o m}_{\mathcal{A}^{\prime}}\left(\mathcal{F} \otimes_{\mathcal{A}} \mathcal{M}, \mathcal{N}\right) \simeq \mathcal{H o m}_{\mathcal{A} \otimes_{\mathcal{R}} \mathcal{A}^{\prime}}\left(\mathcal{M}, \mathcal{H o m} m_{\mathcal{R}}(\mathcal{F}, \mathcal{N})\right)$. Hence 2.16) admits a 
right adjoint, that we denote by $\mathcal{H o m}_{\mathcal{R}}(\mathcal{F}, \cdot)$. This construction is functorial in $\mathcal{F}$, and hence we get the bifunctor $\mathcal{H o m}_{\mathcal{R}}(\cdot, \cdot)$.

The functor $f^{-1}$ was constructed in Proposition 2.14

The functor $f_{*}$ is obtained by noticing that if $\mathfrak{M}$ is a stack of $\mathcal{R}$-twisted modules, then $f^{-1}$ is locally the usual sheaf-theoretical pull-back, which admits a right adjoint.

Assume that $f: Y \rightarrow X$ is a continuous map of locally compact Hausdorff topological spaces. Recall that for an $f^{-1} \mathcal{A}$-module $\mathcal{G}$ on $Y$ one denotes by $f_{!} \mathcal{G}$ the subsheaf of $f_{*} \mathcal{G}$ of sections $s \in f_{*} \mathcal{G}(U)$ such that $\left.f\right|_{\operatorname{supp}(s)}$ is proper. Such a condition is local on $X$, and hence for a stack of $\mathcal{R}$-twisted modules $\mathfrak{M}$ there is an $\mathcal{R}$-functor $f_{!}: f_{*} f^{\circledast} \mathfrak{M} \rightarrow \mathfrak{M}$ locally given by the usual proper direct image functor for sheaves just recalled.

\subsection{Derived twisted operations.}

Let us now deal with the twisted version of Grothendieck's formalism of six operations for sheaves over locally compact Hausdorff topological spaces. We do not recall here such formalism for the classical non-twisted case, referring instead e.g. to 22 .

Let $\mathfrak{M}$ be a stack of $\mathcal{R}$-twisted modules, and denote by $\mathrm{D}(\mathfrak{M})$ the derived category of the abelian category $\mathfrak{M}(X)$. Let $\mathrm{D}^{\mathrm{b}}(\mathfrak{M})\left(\right.$ resp. $\mathrm{D}^{+}(\mathfrak{M})$, resp. $\left.\mathrm{D}^{-}(\mathfrak{M})\right)$ be the full triangulated subcategory of $\mathrm{D}(\mathfrak{M})$ whose objects have bounded (resp. bounded below, resp. bounded above) amplitude.

Lemma 2.18. The category $\mathfrak{M}(X)$ has enough injective objects.

Proof. The classical proof, found e.g. in 22, Proposition 2.4.3], adapts as follows. Consider the natural map $p: \hat{X} \rightarrow X$, where $\hat{X}$ is the set $X$ endowed with the discrete topology. For $F \in \mathfrak{M}(X)$, the adjunction morphism $F \rightarrow p_{*} p^{-1} F$ is injective, and the functor $p_{*}$ is left exact. It is thus enough to find an injection $p^{-1} F \rightarrow I$, where $I$ is an injective object in $p^{\circledast} \mathfrak{M}(\hat{X})$. Since $\hat{X}$ is discrete, $p^{\circledast} \mathfrak{M}$ is equivalent to a stack of (non twisted) modules. ${ }^{8}$

Let $f: Y \rightarrow X$ be a continuous map of topological spaces. Deriving the functors $f^{-1}, f_{*}$, and $\mathcal{H o m}_{\mathcal{R}}$, one gets functors

$$
\begin{aligned}
f^{-1}: \mathrm{D}^{ \pm, \mathrm{b}}(\mathfrak{M}) \rightarrow \mathrm{D}^{ \pm, \mathrm{b}}\left(f^{\circledast} \mathfrak{M}\right), \\
R f_{*}: \mathrm{D}^{+}\left(f^{\circledast} \mathfrak{M}\right) \rightarrow \mathrm{D}^{+}(\mathfrak{M}), \\
R \mathcal{H o m}{ }_{\mathcal{R}}: \mathrm{D}^{-}\left(\mathfrak{M}^{\circledast-1}\right)^{\mathrm{op}} \times \mathrm{D}^{+}\left(\mathfrak{M}^{\prime}\right) \rightarrow \mathrm{D}^{+}\left(\mathfrak{M} \circledast_{\mathcal{R}} \mathfrak{M}^{\prime}\right) .
\end{aligned}
$$

\footnotetext{
${ }^{8}$ Another proof is obtained by applying Grothendieck's criterion, stating that a category has enough injective objects if it admits small filtrant inductive limits, which are exact, and if it admits a generator. Let $\left\{U_{i}\right\}_{i \in I}$ be an open covering of $X$, let $\varphi_{i}:\left.\mathfrak{M o d}\left(\mathcal{A}_{i}\right) \stackrel{\widetilde{\sim}}{\rightarrow} \mathfrak{M}\right|_{U_{i}}$ be $\mathcal{R}$ equivalences for some $\mathcal{R}$-algebras $\mathcal{A}_{i}$, and let $G_{i}$ be generators of $\operatorname{Mod}\left(\mathcal{A}_{i}\right)$. Then a generator of $\mathfrak{M}(X)$ is given by $G=\bigoplus_{i} j_{i !} \varphi_{i}\left(G_{i}\right)$, where $j_{i}: U_{i} \rightarrow X$ are the open inclusions.
} 
Assuming that the weak global dimension of $\mathcal{R}$ is finite, one gets that $\mathfrak{M}(X)$ has enough flat objects. Deriving $\otimes_{\mathcal{R}}$ one gets a functor

$$
\otimes_{\mathcal{R}}^{L}: \mathrm{D}^{ \pm, \mathrm{b}}(\mathfrak{M}) \times \mathrm{D}^{ \pm, \mathrm{b}}\left(\mathfrak{M}^{\prime}\right) \rightarrow \mathrm{D}^{ \pm, \mathrm{b}}\left(\mathfrak{M} \circledast_{\mathcal{R}} \mathfrak{M}^{\prime}\right)
$$

Assuming that $f$ is a map between locally compact Hausdorff topological spaces, one can derive the functor $f_{!}$, and get

$$
R f_{!}: \mathrm{D}^{+}\left(f^{\circledast} \mathfrak{M}\right) \rightarrow \mathrm{D}^{+}(\mathfrak{M})
$$

Assume that $f$ ! has finite cohomological dimension. The usual construction of Poincaré-Verdier duality (cf e.g. [22, §3.1]) extends to the twisted case as follows. Let $L \in \operatorname{Mod}\left(\mathbb{Z}_{X}\right)$, and consider the functor

$$
f_{!}\left(\cdot \otimes_{\mathbb{Z}} L\right): f_{*} f^{\circledast} \mathfrak{M} \rightarrow \mathfrak{M}
$$

Denote by $\mathcal{I}(\mathfrak{M})$ the full substack of $\mathfrak{M}$ of injective objects. Assuming that $L$ is flat and $f$-soft, there exists a functor

$$
f_{L}^{!}: \mathcal{I}(\mathfrak{M}) \rightarrow \mathcal{I}\left(f_{*} f^{\circledast} \mathfrak{M}\right)
$$

characterized by the isomorphism, functorial in $I$ and $G$,

$$
\mathcal{H o m}_{\mathfrak{M}}\left(f_{!}\left(G \otimes_{\mathbb{Z}} L\right), I\right) \simeq f_{*} \mathcal{H} o m_{f^{\circledast \mathfrak{M}}}\left(G, f_{L}^{!} I\right)
$$

In fact, the above isomorphism shows that the existence of $f_{L}^{!}$is a local problem, and locally this is the classical construction. As in the classical case, one finally gets a functor

$$
f^{!}: \mathrm{D}^{+}(\mathfrak{M}) \rightarrow \mathrm{D}^{+}\left(f^{\circledast} \mathfrak{M}\right)
$$

by letting $f^{!} F$ be the simple complex associated to the double complex $f_{L}^{!} \bullet I^{\bullet}$, where $I^{\bullet} \in K^{+}(\mathcal{I}(\mathfrak{M})(X))$ is quasi-isomorphic to $F$, and $L^{\bullet}$ is a (non twisted) bounded, flat, $f$-soft resolution of $\mathbb{Z}_{Y}$.

One proves that the usual formulas relating the six operations above, like adjunction, base-change, or projection formulas, hold.

\section{Descent}

Effective descent data for stacks of twisted modules, called twisting data, are considered in [18, 26, 25], and we recall here this notion using the language of semisimplicial complexes. We then describe in terms of twisting data equivalences, operations, and the example of twisted modules associated with a line bundle. 


\subsection{Morita theory III. Equivalences.}

In Section 2.1 we recalled how functors between stacks of modules admitting an adjoint are described in term of bimodules. We discuss here the particular case of equivalences. (References are again made to [1, 12, 25.)

Two $\mathcal{R}$-algebras $\mathcal{A}$ and $\mathcal{B}$ are called Morita equivalent if $\mathfrak{M o d}(\mathcal{A})$ and $\mathfrak{M o d}(\mathcal{B})$ are $\mathcal{R}$-equivalent. Let us recall how such equivalences are described in terms of $\mathcal{A} \otimes_{\mathcal{R}} \mathcal{B}^{\text {op }}$-modules.

Proposition 3.1. Let $\mathcal{L}$ be an $\mathcal{A} \otimes_{\mathcal{R}} \mathcal{B}^{\text {op }}$-module. Then the following conditions are equivalent:

(i) There exists a $\mathcal{B} \otimes_{\mathcal{R}} \mathcal{A}^{\mathrm{op}}$-module $\mathcal{L}^{\prime}$, such that $\mathcal{L} \otimes_{\mathcal{B}} \mathcal{L}^{\prime} \simeq \mathcal{A}$ as $\mathcal{A} \otimes_{\mathcal{R}} \mathcal{A}^{\mathrm{op}}$ modules and $\mathcal{L}^{\prime} \otimes_{\mathcal{A}} \mathcal{L} \simeq \mathcal{B}$ as $\mathcal{B} \otimes_{\mathcal{R}} \mathcal{B}^{\text {op }}$-modules.

(ii) For $\mathcal{L}^{* \mathcal{A}}=\mathcal{H o m}_{\mathcal{A}}(\mathcal{L}, \mathcal{A})$, the canonical morphism $\mathcal{L} \otimes_{\mathcal{B}} \mathcal{L}^{* \mathcal{A}} \rightarrow \mathcal{A}$ is an isomorphism of $\mathcal{A} \otimes_{\mathcal{R}} \mathcal{A}^{\text {op }}$-modules, and $\mathcal{L}^{* \mathcal{A}} \otimes_{\mathcal{A}} \mathcal{L} \simeq \mathcal{B}$ as $\mathcal{B} \otimes_{\mathcal{R}} \mathcal{B}^{\text {op }}$-modules.

(iii) $\mathcal{L}$ is a faithfully flat $\mathcal{A}$-module locally of finite presentation, and there is an $\mathcal{R}$-algebra isomorphism $\mathcal{B}^{\text {op }} \stackrel{\sim}{\rightarrow} \mathcal{E} d_{\mathcal{A}}(\mathcal{L})$.

(iv) $\mathcal{L}$ is a faithfully flat $\mathcal{B}^{\mathrm{op}}$-module locally of finite presentation, and there is an $\mathcal{R}$-algebra isomorphism $\mathcal{A} \stackrel{\sim}{\rightarrow} \mathcal{E} n d_{\mathcal{B}^{\circ \mathrm{p}}}(\mathcal{L})$.

(v) $\mathcal{L} \otimes_{\mathcal{B}}(\cdot): \mathfrak{M o d}(\mathcal{B}) \rightarrow \mathfrak{M o d}(\mathcal{A})$ is an $\mathcal{R}$-equivalence.

(vi) $\mathcal{H o m}_{\mathcal{A}}(\mathcal{L}, \cdot): \mathfrak{M o d}(\mathcal{A}) \rightarrow \mathfrak{M o d}(\mathcal{B})$ is an $\mathcal{R}$-equivalence.

Definition 3.2. An $\mathcal{A} \otimes_{\mathcal{R}} \mathcal{B}^{\text {op }}$-module $\mathcal{L}$ is called invertible if the equivalent conditions in Proposition 3.1 are satisfied.

The ring $\mathcal{A}$ itself is the invertible $\mathcal{A} \otimes_{\mathcal{R}} \mathcal{A}^{\mathrm{op}}$-module corresponding to the identity functor of $\mathfrak{M o d}(\mathcal{A})$. Note that invertible $\mathcal{A} \otimes_{\mathcal{R}} \mathcal{A}^{\mathrm{op}}$-modules are not necessarily locally isomorphic to $\mathcal{A}$ as $\mathcal{A}$-modules, even if $\mathcal{A}$ is a commutative ring.

Theorem 3.3 (Morita). If $\varphi: \mathfrak{M o d}(\mathcal{B}) \rightarrow \mathfrak{M o d}(\mathcal{A})$ is an equivalence of $\mathcal{R}$-stacks, then $\mathcal{L}=\varphi(\mathcal{B})$ is an invertible $\mathcal{A} \otimes_{\mathcal{R}} \mathcal{B}^{\mathrm{op}}$-module, and $\varphi \simeq \mathcal{L} \otimes_{\mathcal{B}}(\cdot)$. Moreover, a quasi-inverse to $\varphi$ is given by $\operatorname{Hom}_{\mathcal{A}}(\mathcal{L}, \cdot) \simeq \mathcal{L}^{* \mathcal{A}} \otimes_{\mathcal{A}}(\cdot)$.

Proof. Let $\psi$ be a quasi-inverse to $\varphi$. Since $\psi$ is right adjoint to $\varphi$, by Proposition $2.1 \mathcal{L}=\varphi(\mathcal{B})$ is an $\mathcal{A} \otimes_{\mathcal{R}} \mathcal{B}^{\text {op }}$-module such that $\varphi \simeq \mathcal{L} \otimes_{\mathcal{B}}(\cdot)$. Interchanging the role of $\varphi$ and $\psi$ there also exists a $\mathcal{B} \otimes_{\mathcal{R}} \mathcal{A}^{\text {op }}$-module $\mathcal{L}^{\prime}$ such that $\psi \simeq \mathcal{L}^{\prime} \otimes_{\mathcal{A}}(\cdot)$. Since $\varphi \circ \psi$ and $\psi \circ \varphi$ are isomorphic to the identity functors, $\mathcal{L}$ is invertible and $\mathcal{L}^{\prime} \simeq \mathcal{L}^{* \mathcal{A}}$. Finally, since $\mathcal{L}$ is a flat $\mathcal{A}$-module locally of finite presentation, one has $\mathcal{L}^{* \mathcal{A}} \otimes_{\mathcal{A}}(\cdot) \simeq \mathcal{H o m}_{\mathcal{A}}(\mathcal{L}, \cdot)$. 


\subsection{Twisting data on an open covering.}

By definition, if $\mathfrak{M}$ is a stack of $\mathcal{R}$-twisted modules there exist an open covering $\left\{U_{i}\right\}_{i \in I}$ of $X,\left.\mathcal{R}\right|_{U_{i}}$-algebras $\mathcal{A}_{i}$ on $U_{i}$, and $\left.\mathcal{R}\right|_{U_{i}}$-equivalences of $\left.\mathcal{R}\right|_{U_{i}}$-stacks $\varphi_{i}:\left.\mathfrak{M}\right|_{U_{i}} \rightarrow \mathfrak{M o d}\left(\mathcal{A}_{i}\right)$. Let $\psi_{i}$ be a quasi-inverse of $\varphi_{i}$, and let $\alpha_{i}: \psi_{i} \circ \varphi_{i} \Rightarrow \operatorname{id}_{\mathfrak{M}}$ be an invertible transformation. By (the $\mathcal{R}$-linear analogue of) Proposition 1.6 the following descent datum for stacks is enough to reconstruct $\mathfrak{M}$

$$
\left(\left\{U_{i}\right\}_{i \in I},\left\{\mathfrak{M o d}\left(\mathcal{A}_{i}\right)\right\}_{i \in I},\left\{\varphi_{i j}\right\}_{i, j \in I},\left\{\alpha_{i j k}\right\}_{i, j, k \in I}\right) .
$$

Here $\varphi_{i j}=\left.\left.\varphi_{i}\right|_{U_{i j}} \circ \psi_{j}\right|_{U_{i j}}$, and $\alpha_{i j k}: \varphi_{i j} \circ \varphi_{j k} \Rightarrow \varphi_{i k}$ is induced by $\alpha_{j}$, so that they satisfy condition (1.3). Functors as $\varphi_{i j}$ are described by Morita's Theorem 3.3 so that the descent datum (3.1) is replaced by

$$
\mathrm{t}=\left(\left\{U_{i}\right\}_{i \in I},\left\{\mathcal{A}_{i}\right\}_{i \in I},\left\{\mathcal{L}_{i j}\right\}_{i, j \in I},\left\{a_{i j k}\right\}_{i, j, k \in I}\right),
$$

where $\left\{U_{i}\right\}_{i \in I}$ is an open covering of $X, \mathcal{A}_{i}$ is an $\left.\mathcal{R}\right|_{U_{i}}$-algebra on $U_{i}, \mathcal{L}_{i j}$ are invertible $\left.\mathcal{A}_{i} \otimes_{\mathcal{R}} \mathcal{A}_{j}^{\text {op }}\right|_{U_{i j}}$-modules, and $a_{i j k}:\left.\left.\mathcal{L}_{i j} \otimes_{\mathcal{A}_{j}} \mathcal{L}_{j k}\right|_{U_{i j k}} \rightarrow \mathcal{L}_{i k}\right|_{U_{i j k}}$ are isomorphisms of $\left.\mathcal{A}_{i} \otimes_{\mathcal{R}} \mathcal{A}_{k}^{\text {op }}\right|_{U_{i j k}}$-modules satisfying the analogue of condition (1.3). As in the proof of Proposition 1.6 up to equivalence a twisted module $\mathcal{F} \in \mathfrak{M}(X)$ is thus described by a pair

$$
\left(\left\{\mathcal{F}_{i}\right\}_{i \in I},\left\{m_{i j}\right\}_{i, j \in I}\right),
$$

where $\mathcal{F}_{i} \in \operatorname{Mod}\left(\mathcal{A}_{i}\right)$, and $m_{i j}:\left.\left.\mathcal{L}_{i j} \otimes_{\mathcal{A}_{j}} \mathcal{F}_{j}\right|_{U_{i j}} \rightarrow \mathcal{F}_{i}\right|_{U_{i j}}$ is an isomorphism of $\left.\mathcal{A}_{i}\right|_{U_{i j}}$-modules on $U_{i j}$ such that the following diagram on $U_{i j k}$ commutes

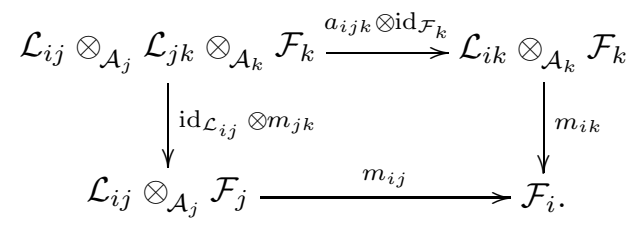

This is actually the definition of twisted modules given in [18. It is also an example of twisting data, of which we now give a more general definition.

\subsection{Twisting data.}

We shall use here the language of semisimplicial complexes. On the one hand, this allows one to consider more general situations than open coverings, on the other hand, it provides a very efficient bookkeeping of indices.

Recall that semisimplicial complexes are diagrams of continuous maps of topological spaces ${ }^{9}$

$$
X^{[3]} \stackrel{q_{0}^{[3]}, \ldots, q_{3}^{[3]}}{\Longrightarrow} X^{[2]} \stackrel{q_{0}^{[2]}, q_{1}^{[2]}, q_{2}^{[2]}}{\Longrightarrow} X^{[1]} \stackrel{q_{0}^{[1]}, q_{1}^{[1]}}{\Longrightarrow} X^{[0]} \stackrel{q_{0}^{[0]}=q^{[0]}=q}{\longrightarrow} X^{[-1]}=X,
$$

\footnotetext{
${ }^{9}$ In dealing with stacks, we will only need the terms $X^{[r]}$ with $r \leq 3$.
} 
satisfying the commutativity relations

$$
q_{j}^{[r]} \circ q_{i}^{[r+1]}=q_{i}^{[r]} \circ q_{j+1}^{[r+1]},
$$

for $0 \leq i \leq j \leq r$. In the coskeleton construction, one considers the topological space

$$
X^{[r]+1}=\left\{\left(x_{0}, \ldots, x_{r+1}\right) \in\left(X^{[r]}\right)^{r+2}: q_{j}^{[r]}\left(x_{i}\right)=q_{i}^{[r]}\left(x_{j+1}\right) \text { for } 0 \leq i \leq j \leq r\right\},
$$

and let $q^{[r+1]}: X^{[r+1]} \rightarrow X^{[r]+1}$ be the map $x \mapsto\left(q_{0}^{[r+1]}(x), \ldots, q_{r+1}^{[r+1]}(x)\right)$. Hence there are commutative diagrams for $0 \leq i \leq r+1$

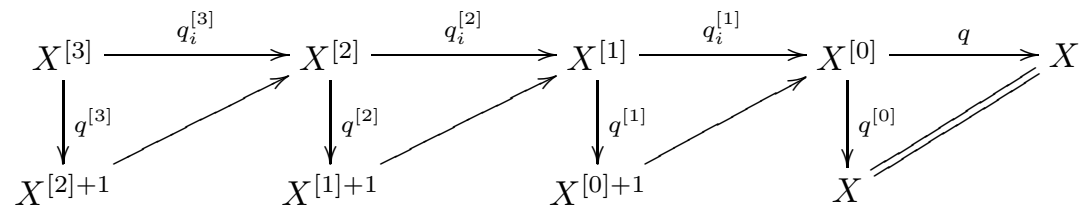

where the diagonal arrows are the projection to the $i$ th factor.

Example 3.4. (a) Let us say that a semisimplicial complex is coskeletal if $X^{[r+1]} \simeq X^{[r]+1}$ for $r \geq 0$. In other words, $X^{[r]}=Y \times_{X} \cdots \times_{X} Y$ is the $(r+1)$-fold fibered product of a continuous map $q: Y \rightarrow X$, and $q_{i}^{[r]}$ the projection omitting the $i$ th factor.

(a1) A particular case of coskeletal semisimplicial complex is the one attached to an open covering $\left\{U_{i}\right\}_{i \in I}$ of $X$. In this case, $Y=\bigsqcup_{i \in I} U_{i}$ is the disjoint union of the $U_{i}$ 's, and $q$ is the natural map (which is a local homeomorphism). Note that $X^{[r]}=\bigsqcup_{i_{0}, \ldots, i_{r} \in I} U_{i_{0} \cdots i_{r}}$.

(a2) Another particular case of coskeletal semisimplicial complex is obtained when $q: Y \rightarrow X$ is a principal $G$-bundle, for $G$ a topological group. Denoting by $m: G \times Y \rightarrow Y$ the group action, this semisimplicial complex is identified with

$$
G \times G \times G \times Y \stackrel{q_{i}^{[3]}}{\Longrightarrow} G \times G \times Y \stackrel{q_{i}^{[2]}}{\Longrightarrow} G \times Y \stackrel{q_{i}^{[1]}}{ }=Y \stackrel{q}{\longrightarrow} X
$$

where $q_{r}^{[r]}=\operatorname{id}_{G^{r-1}} \times m, q_{0}^{[r]}$ is the projection omitting the 0th factor, and $q_{i}^{[r]}\left(g_{0}, \ldots, g_{r-1}, y\right)=\left(g_{0}, \ldots, g_{i-1} g_{i}, \ldots, g_{r-1}, y\right)$ for $0 \leq i<r$.

(b) Other examples of semisimplicial complexes are the ones attached to hypercoverings, where $X^{[r+1]}$ is induced by an open covering of $X^{[r]+1}$. These are of the form $X^{[0]}=\bigsqcup_{i} U_{i}$ for $X=\bigcup_{i \in I} U_{i}, X^{[1]}=\bigsqcup_{i, j, \alpha} U_{i j}^{\alpha}$, for $U_{i j}=$ $\bigcup_{\alpha \in A_{i j}} U_{i j}^{\alpha}, X^{[2]}=\bigsqcup_{i, j, k, \alpha, \beta, \gamma, \xi} U_{i j k \alpha \beta \gamma}^{\xi}$ for $U_{i j}^{\alpha} \cap U_{k j}^{\beta} \cap U_{k i}^{\gamma}=\bigcup_{\xi \in \Xi_{i j k}^{\alpha \beta \gamma}} U_{i j k \alpha \beta \gamma}^{\xi}$, and so on.

Let $s>r, 0 \leq i_{0}<\cdots<i_{r} \leq s$, and $0 \leq i_{r+1}<\cdots<i_{s} \leq s$, be such that $\left\{i_{0}, \ldots, i_{s}\right\}=\{0, \ldots, s\}$. If $\mathcal{F}$ is a sheaf on $X^{[r]}$, we denote by $\mathcal{F}_{i_{0} \cdots i_{r}}=$ 
$\left(q_{i_{r+1}}^{[r+1]} \circ \cdots \circ q_{i_{s}}^{[s]}\right)^{-1} \mathcal{F}$ its sheaf-theoretical pull-back to $X^{[s]}$, and we use the same notations for morphisms of sheaves. ${ }^{10}$

Definition 3.5. (i) An $\mathcal{R}$-twisting datum on $X$ is a quadruplet ${ }^{11}$

$$
\mathrm{t}=\left(X^{[\bullet]} \stackrel{q}{\rightarrow} X, \mathcal{A}, \mathcal{L}, a\right),
$$

where $X^{[\bullet]} \stackrel{q}{\rightarrow} X$ is a semisimplicial complex, $\mathcal{A}$ is a $q^{-1} \mathcal{R}$-algebra on $X^{[0]}, \mathcal{L}$ is an invertible $\mathcal{A}_{0} \otimes_{\mathcal{R}} \mathcal{A}_{1}^{\text {op }}$-module on $X^{[1]}$, and $a: \mathcal{L}_{01} \otimes_{\mathcal{A}_{1}} \mathcal{L}_{12} \rightarrow \mathcal{L}_{02}$ is an isomorphism of $\mathcal{A}_{0} \otimes_{\mathcal{R}} \mathcal{A}_{2}^{\text {op }}$-modules on $X^{[2]}$ such that the following diagram on $X^{[3]}$ commutes $^{12}$

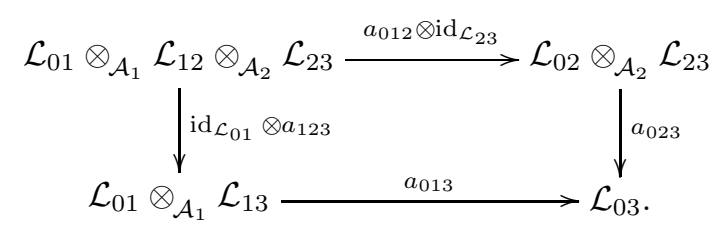

(ii) A coskeletal $\mathcal{R}$-twisting datum on $X$ is an $\mathcal{R}$-twisting datum whose associated semisimplicial complex is coskeletal.

One can now mimic the construction in the sketch of proof of Proposition 1.6 Denote by $\operatorname{Mod}(\mathrm{t})$ the category whose objects are pairs $(\mathcal{F}, m)$, where $\mathcal{F}$ is an $\mathcal{A}$-module on $X^{[0]}$, and $m: \mathcal{L} \otimes_{\mathcal{A}_{1}} \mathcal{F}_{1} \rightarrow \mathcal{F}_{0}$ is an isomorphism of $\mathcal{A}_{0}$-modules on

\footnotetext{
${ }^{10}$ In the coskeletal case, $\mathcal{F}_{i_{0} \cdots i_{r}}$ is the pull-back of $\mathcal{F}$ by the projection to the $\left(i_{0}, \ldots, i_{r}\right)$ th factors.

${ }^{11}$ This notion was discussed in 18 for semisimplicial complexes attached to open coverings, and in [26] for coskeletal semisimplicial complexes.

${ }^{12}$ Let us denote by $\mathcal{L}_{i j}^{[r]}$ the sheaf $\mathcal{L}_{i j}$ on $X^{[r]}$. Then one should pay attention to the fact that in $X^{[3]}$ one has isomorphisms like $\mathcal{L}_{01}^{[3]} \simeq\left(q_{3}^{[3]}\right)^{-1} \mathcal{L}_{01}^{[2]}$, but not equalities. Thus, much as in Definition 1.4 (iv), one should write 3.5 more precisely as

$$
\begin{aligned}
& \left(q_{3}^{[3]}\right)^{-1}\left(\mathcal{L}_{01}^{[2]} \otimes_{\mathcal{A}_{1}} \mathcal{L}_{12}^{[2]}\right) \otimes_{\mathcal{A}_{2}} \mathcal{L}_{23}^{[3]} \simeq \mathcal{L}_{01}^{[3]} \otimes_{\mathcal{A}_{1}} \mathcal{L}_{12}^{[3]} \otimes_{\mathcal{A}_{2}} \mathcal{L}_{23}^{[3]} \simeq \mathcal{L}_{01}^{[3]} \otimes_{\mathcal{A}_{1}}\left(q_{0}^{[3]}\right)^{-1}\left(\mathcal{L}_{01}^{[2]} \otimes_{\mathcal{A}_{1}} \mathcal{L}_{12}^{[2]}\right) \\
& { }^{a \otimes \mathrm{id}}{ }_{\mathcal{L}_{23}^{[3]} \downarrow} \\
& \left(q_{3}^{[3]}\right)^{-1} \mathcal{L}_{02}^{[2]} \otimes_{\mathcal{A}_{2}} \mathcal{L}_{23}^{[3]} \\
& \sim 1 \\
& \mathcal{L}_{02}^{[3]} \otimes_{\mathcal{A}_{2}} \mathcal{L}_{23}^{[3]} \\
& \sim 1 \\
& \left(q_{1}^{[3]}\right)^{-1}\left(\mathcal{L}_{01}^{[2]} \otimes_{\mathcal{A}_{1}} \mathcal{L}_{12}^{[2]}\right) \\
& a \downarrow \\
& { }_{\text {id }} \mathcal{L}_{01}^{[3]} \otimes a \downarrow \\
& \mathcal{L}_{01}^{[3]} \otimes_{\mathcal{A}_{1}}\left(q_{0}^{[3]}\right)^{-1} \mathcal{L}_{02}^{[2]} \\
& \sim 1 \\
& \mathcal{L}_{01}^{[3]} \otimes_{\mathcal{A}_{1}} \mathcal{L}_{13}^{[3]} \\
& \sim 1 \\
& \left(q_{2}^{[3]}\right)^{-1}\left(\mathcal{L}_{01}^{[2]} \otimes_{\mathcal{A}_{1}} \mathcal{L}_{12}^{[2]}\right) \\
& a \downarrow \\
& \mathcal{L}_{03}^{[3]} \sim\left(q_{2}^{[3]}\right)^{-1} \mathcal{L}_{02}^{[2]} .
\end{aligned}
$$$$
\left(q_{1}^{[3]}\right)^{-1} \mathcal{L}_{02}^{[2]} \sim \mathcal{L}_{03}^{[3}
$$

Such a level of precision is both quite cumbersome and easy to attain, so we prefer a sloppier but clearer presentation. 
$X^{[1]}$ such that the following diagram on $X^{[2]}$ commutes

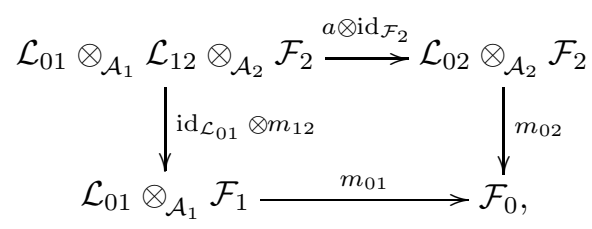

and whose morphisms $\alpha:(\mathcal{F}, m) \rightarrow\left(\mathcal{F}^{\prime}, m^{\prime}\right)$ consists of morphisms of $\mathcal{A}$-modules $\alpha: \mathcal{F} \rightarrow \mathcal{F}^{\prime}$, such that the following diagram on $X^{[1]}$ commutes

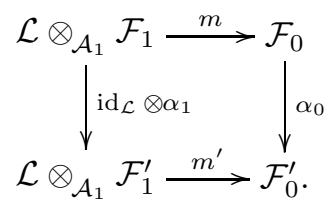

Definition 3.6. Let $\mathrm{t}$ be an $\mathcal{R}$-twisting datum on $X$. We denote by $\mathfrak{M o d}(\mathrm{t})$ the prestack on $X$ defined by $U \mapsto \operatorname{Mod}\left(\left.\mathrm{t}\right|_{U}\right)$, which is in fact an $\mathcal{R}$-stack. Here, $\left.\mathrm{t}\right|_{U}$ denotes the $\left.\mathcal{R}\right|_{U}$-twisting datum on $U$ naturally induced by $\mathrm{t}$.

Note that if $\mathcal{B}$ is an $\mathcal{R}$-algebra on $X$, then $\mathfrak{M o d}(\mathcal{B}) \approx \mathfrak{M o d}\left(1_{\mathcal{B}}\right)$ for

$$
1_{\mathcal{B}}=(X \stackrel{\text { id }}{\rightarrow} X, \mathcal{B}, \mathcal{B}, \cdot)
$$

the trivial $\mathcal{R}$-twisting datum, with $\cdot$ being the canonical isomorphism $\mathcal{B} \otimes_{\mathcal{B}} \mathcal{B} \stackrel{\sim}{\rightarrow} \mathcal{B}$.

We spend the rest of this section to show that $\mathfrak{M o d}(\mathrm{t})$ is actually a stack of $\mathcal{R}$ twisted modules, using arguments adapted from those in [26]. In order to get this result it seems natural to assume that the maps $q^{[r]}: X^{[r]} \rightarrow X^{[r-1]+1}$ admit local sections for $r=0,1,2,3$. However, we will consider here the stronger assumption

$$
\text { the maps } q^{[r]} \text {, for } r=0,1,2,3 \text {, admit sections locally on } X \text {. }
$$

Note that for coskeletal semisimplicial complexes this reduces to the assumption

$$
q: X^{[0]} \rightarrow X \text { admits local sections, }
$$

which holds true for semisimplicial complexes attached to open coverings or to principal $G$-bundles, as in Example 3.4 (a1) and (a2). In general, (3.7) does not hold for semisimplicial complexes attached to hypercoverings, as in Example 3.4 (b).

Let $\mathrm{t}^{\prime}=\left(X^{\prime[\bullet]} \stackrel{q^{\prime}}{\rightarrow} X, \mathcal{A}^{\prime}, \mathcal{L}^{\prime}, a^{\prime}\right)$ be another $\mathcal{R}$-twisting datum on $X$. 
Definition 3.7. (i) A refinement of $\mathcal{R}$-twisting data $\rho: \mathrm{t}^{\prime} \rightarrow \mathrm{t}$ consists of commutative diagrams

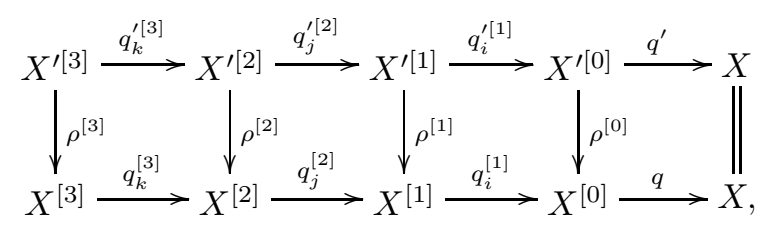

of an isomorphism of $q^{-1} \mathcal{R}$-algebras $\left(\rho^{[0]}\right)^{-1} \mathcal{A} \stackrel{\sim}{\rightarrow} \mathcal{A}^{\prime}$, and of an isomorphism of $\mathcal{A}_{0}^{\prime} \otimes_{\mathcal{R}} \mathcal{A}_{1}^{\text {op }}$-modules $\left(\rho^{[1]}\right)^{-1} \mathcal{L} \stackrel{\sim}{\rightarrow} \mathcal{L}^{\prime}$ compatible with $a$ and $a^{\prime}$.

(ii) To $\rho: \mathrm{t}^{\prime} \rightarrow \mathrm{t}$ one associates the functor $\rho^{-1}: \mathfrak{M} \mathfrak{o d}(\mathrm{t}) \rightarrow \mathfrak{M o d}\left(\mathrm{t}^{\prime}\right)$, given by $(\mathcal{F}, m) \mapsto\left(\left(\rho^{[0]}\right)^{-1} \mathcal{F},\left(\rho^{[1]}\right)^{-1} m\right)$.

Lemma 3.8. Let $\mathrm{t}=\left(X^{[\bullet]} \stackrel{q}{\rightarrow} X, \mathcal{A}, \mathcal{L}, a\right)$ be such that the maps $q^{[r]}$ admit global sections $s^{[r]}$. Then

(i) there is a refinement of $\mathcal{R}$-twisting data $\tilde{s}: 1_{\left(s^{[0]}\right)^{-1} \mathcal{A}} \rightarrow \mathrm{t}$,

(ii) the functor $\tilde{s}^{-1}: \mathfrak{M o d}(\mathrm{t}) \rightarrow \mathfrak{M o d}\left(\left(s^{[0]}\right)^{-1} \mathcal{A}\right)$ is an equivalence.

Proof. Define the maps $\tilde{s}^{[r]}: X \rightarrow X^{[r]}$ by induction as ${ }^{13}$

$$
\tilde{s}^{[0]}(x)=s^{[0]}(x), \quad \tilde{s}^{[r+1]}(x)=s^{[r+1]}\left(\tilde{s}^{[r]}(x), \ldots, \tilde{s}^{[r]}(x)\right) .
$$

Since $q_{i}^{[2]} \circ \tilde{s}^{[2]}=\tilde{s}^{[1]}$, one has isomorphisms $\left(\tilde{s}^{[2]}\right)^{-1} \mathcal{L}_{j k} \simeq\left(\tilde{s}^{[1]}\right)^{-1} \mathcal{L}$. Then, $a$ gives an isomorphism

$$
\left(\tilde{s}^{[1]}\right)^{-1} \mathcal{L} \otimes_{\left(s^{[0]}\right)^{-1} \mathcal{A}}\left(\tilde{s}^{[1]}\right)^{-1} \mathcal{L} \simeq\left(\tilde{s}^{[1]}\right)^{-1} \mathcal{L} .
$$

Since $\mathcal{L}$ is invertible, there is an $\mathcal{A}_{1} \otimes_{\mathcal{R}} \mathcal{A}_{0}^{\text {op }}$-module $\mathcal{L}^{\prime}$ such that $\mathcal{L} \otimes_{\mathcal{A}_{1}} \mathcal{L}^{\prime} \simeq \mathcal{A}_{0}$. Applying the functor $(\cdot) \otimes_{\left(s^{[0]}\right)^{-1} \mathcal{A}}\left(\tilde{s}^{[1]}\right)^{-1} \mathcal{L}^{\prime}$ to (3.9), we get an isomorphism of $\left(s^{[0]}\right)^{-1} \mathcal{A} \otimes_{\mathcal{R}}\left(s^{[0]}\right)^{-1} \mathcal{A}^{\mathrm{op}}$-modules $\left(\tilde{s}^{[1]}\right)^{-1} \mathcal{L} \simeq\left(s^{[0]}\right)^{-1} \mathcal{A}$. This proves (i).

To prove (ii), let us define the maps $\sigma^{[r]}: X^{[r]} \rightarrow X^{[r+1]}$ by induction as ${ }^{14}$

$$
\sigma^{[-1]}=s^{[0]}, \quad \sigma^{[r]}(x)=s^{[r+1]}\left(\sigma^{[r-1]}\left(q_{0}^{[r]}(x)\right), \ldots, \sigma^{[r-1]}\left(q_{r}^{[r]}(x)\right), x\right) .
$$

Using the maps $\sigma^{[r]}$ one gets a functor $\sigma^{-1}: \mathfrak{M o d}\left(\left(s^{[0]}\right)^{-1} \mathcal{A}\right) \rightarrow \mathfrak{M o d}(\mathrm{t})$, given by $\mathcal{G} \mapsto\left(\left(\sigma^{[0]}\right)^{-1} \mathcal{L} \otimes_{q^{-1}\left(s^{[0]}\right)^{-1} \mathcal{A}} q^{-1} \mathcal{G},\left(\sigma^{[1]}\right)^{-1} a\right)$. This is well-defined, since [3.6] is obtained by applying $\left(\sigma^{[2]}\right)^{-1}$ to (3.5). One checks that $\sigma^{-1}$ is a quasi-inverse to $\tilde{s}^{-1}$.

Proposition 3.9. (i) Let $\mathrm{t}$ be an $\mathcal{R}$-twisting datum on $X$ satisfying (3.7). Then $\mathfrak{M o d}(\mathrm{t})$ is a stack of $\mathcal{R}$-twisted modules.

\footnotetext{
${ }^{13}$ For coskeletal semisimplicial complexes, one has $\tilde{s}^{[r]}=\delta^{[r]} \circ s^{[0]}$, where $\delta^{[r]}: X^{[0]} \rightarrow X^{[r]}$ is the diagonal embedding.

${ }^{14}$ For coskeletal semisimplicial complexes, one has $\sigma^{[r]}(x)=\left(x, s^{[0]}(q(x))\right) \in X^{[r]} \times{ }_{X} X^{[0]}=$ $X^{[r+1]}$, where $q: X^{[r]} \rightarrow X$ is the composite of the $q_{i}^{[j]}$, s maps.
} 
(ii) Any stack of $\mathcal{R}$-twisted modules on $X$ is $\mathcal{R}$-equivalent to $\mathfrak{M}$ od $(\mathrm{t})$ for some coskeletal $\mathcal{R}$-twisting datum t satisfying (3.8).

Proof. By definition, the maps $q^{[r]}$ 's admit local sections on $X$. Hence part (i) follows from Lemma 3.8 As for (ii), it is enough to take $t$ as in (3.2).

Proposition 3.10. Let $\rho: \mathrm{t}^{\prime} \rightarrow \mathrm{t}$ be a refinement of coskeletal $\mathcal{R}$-twisting data on $X$ satisfying (3.8). Then the functor $\rho^{-1}: \mathfrak{M o d}(\mathrm{t}) \rightarrow \mathfrak{M o d}\left(\mathrm{t}^{\prime}\right)$ is an $\mathcal{R}$-equivalence.

Proof. Let $\mathrm{t}=\left(X^{[\bullet]} \stackrel{q}{\rightarrow} X, \mathcal{A}, \mathcal{L}, a\right)$ and $\mathrm{t}^{\prime}=\left(X^{\prime[\bullet} \stackrel{q^{\prime}}{\rightarrow} X, \mathcal{A}^{\prime}, \mathcal{L}^{\prime}, a^{\prime}\right)$. Proving that $\rho^{-1}$ is an equivalence is a local problem, and we may thus assume that $q^{\prime}: X^{\prime[0]} \rightarrow X$ admits a global section. Then $s=\rho^{[0]} \circ s^{\prime}$ is a global section of $q: X^{[0]} \rightarrow X$. With the notations of Lemma 3.8 one has $\tilde{s}=\rho \circ \tilde{s}^{\prime}$. Hence there is a diagram of functors commuting up to an invertible transformation

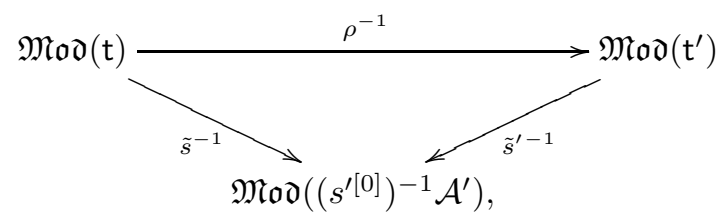

whose diagonal arrows are equivalences.

\subsection{Classification of stacks of twisted modules.}

One may consider coskeletal $\mathcal{R}$-twisting data $\mathrm{t}=\left(X^{[\bullet]} \stackrel{q}{\rightarrow} X, \mathcal{A}, \mathcal{L}, a\right)$ as a kind of Cech cocycles attached to the covering $q$, with (3.5) playing the role of the cocycle condition. There is also a straightforward analogue to the notion of coboundary, given by Morita theorem as follows. Let $\mathrm{u}=\left(X^{[\bullet]} \stackrel{q}{\rightarrow} X, \mathcal{B}, \mathcal{M}, b\right)$ be another coskeletal $\mathcal{R}$-twisting datum attached to the same covering $q$ as t. Let us say that $\mathrm{t}$ and $\mathrm{u}$ differ by a coboundary if there exist a pair $(\mathcal{E}, e)$ where $\mathcal{E}$ is an invertible $\mathcal{A} \otimes_{\mathcal{R}} \mathcal{B}^{\text {op }}$-module on $X^{[0]}$, and $e: \mathcal{L} \otimes_{\mathcal{A}_{1}} \mathcal{E}_{1} \stackrel{\sim}{\rightarrow} \mathcal{E}_{0} \otimes_{\mathcal{B}_{0}} \mathcal{M}$ is an isomorphism of $\mathcal{A}_{0} \otimes_{\mathcal{R}} \mathcal{B}_{1}^{\text {op }}$-modules on $X^{[1]}$ such that the following diagram on $X^{[2]}$ commutes

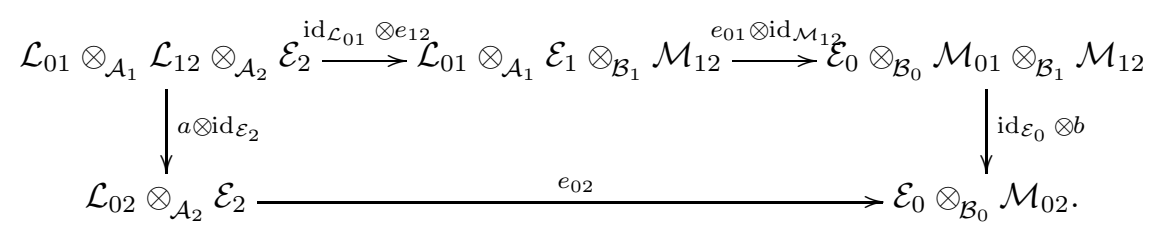

In this case, there is an $\mathcal{R}$-equivalence $\mathfrak{M} \mathfrak{l o d}(\mathrm{u}) \rightarrow \mathfrak{M o d}(\mathrm{t})$ given by $(\mathcal{G}, n) \mapsto$ $\left(\mathcal{E} \otimes_{\mathcal{B}} \mathcal{G},\left(\operatorname{id}_{\mathcal{E}_{0}} \otimes n\right) \circ\left(e \otimes \operatorname{id}_{\mathcal{G}_{1}}\right)\right)$.

Note that $\mathcal{R}$-equivalence classes of stacks of $\mathcal{R}$-twisted modules are in oneto-one correspondence with this "cohomology". (The analogue correspondence appears in [32 for the case of bundle gerbes, and in [6] for general gerbes.) In fact, one checks that if $\mathrm{t}=\left(X^{[\bullet]} \stackrel{q}{\rightarrow} X, \mathcal{A}, \mathcal{L}, a\right)$ and $\mathrm{u}=\left(Y^{[\bullet]} \stackrel{r}{\rightarrow} X, \mathcal{B}, \mathcal{M}, b\right)$ are 
arbitrary coskeletal $\mathcal{R}$-twisting data, then $\mathfrak{M o d}(\mathrm{t})$ and $\mathfrak{M o d}(\mathrm{u})$ are $\mathcal{R}$-equivalent if and only if $t$ and $u$ differ by a coboundary on a common refinement. This means that there exist refinements of coskeletal $\mathcal{R}$-twisting data $\mathrm{t}^{\prime} \rightarrow \mathrm{t}$ and $\mathrm{u}^{\prime} \rightarrow \mathrm{u}$ such that $\mathrm{t}^{\prime}$ and $\mathrm{u}^{\prime}$ are attached to the same covering, and differ by a coboundary.

\subsection{Operations in terms of twisting data.}

Operations for stacks of twisted $\mathbb{C}$-modules were described in [26] using twisting data. We give here a similar description for general twisted modules.

Let $\mathrm{t}=\left(X^{[\bullet]} \stackrel{q}{\rightarrow} X, \mathcal{A}, \mathcal{L}, a\right)$ be an $\mathcal{R}$-twisting datum on the topological space $X$. Its opposite is the $\mathcal{R}$-twisting datum

$$
\mathrm{t}^{\mathrm{op}}=\left(X^{[\bullet]} \stackrel{q}{\rightarrow} X, \mathcal{A}^{\mathrm{op}}, \mathcal{L}^{-1}, a^{-1}\right),
$$

where $\mathcal{L}^{-1}=\mathcal{H o m}_{\mathcal{A}_{0}}\left(\mathcal{L}, \mathcal{A}_{0}\right)$, and $a^{-1}$ is the inverse of the following chain of isomorphisms

$$
\begin{aligned}
\mathcal{L}_{02}^{-1} & =\mathcal{H o m}_{\mathcal{A}_{0}}\left(\mathcal{L}_{02}, \mathcal{A}_{0}\right) \\
& \stackrel{a}{\rightarrow} \mathcal{H o m}_{\mathcal{A}_{0}}\left(\mathcal{L}_{01} \otimes_{\mathcal{A}_{1}} \mathcal{L}_{12}, \mathcal{A}_{0}\right) \\
& \simeq \mathcal{H o m}{ }_{\mathcal{A}_{1}}\left(\mathcal{L}_{12}, \mathcal{H} \mathrm{Hom}_{\mathcal{A}_{0}}\left(\mathcal{L}_{01}, \mathcal{A}_{0}\right)\right) \\
& \simeq \mathcal{H o m} \\
& =\mathcal{L}_{\mathcal{A}_{1}}\left(\mathcal{L}_{12}, \mathcal{A}_{1}\right) \otimes_{\mathcal{A}_{1}} \mathcal{H o m}_{\mathcal{A}_{0}}\left(\mathcal{L}_{01}, \mathcal{A}_{0}\right) \\
\otimes_{\mathcal{A}_{1}^{\mathrm{op}}} & \mathcal{L}_{12}^{-1},
\end{aligned}
$$

where in the last isomorphism holds because $\mathcal{L}_{12}$ is a flat $\mathcal{A}_{1}$-module locally of finite presentation.

Let $\mathrm{t}^{\prime}=\left(X^{\prime[\bullet]} \stackrel{q^{\prime}}{\rightarrow} X, \mathcal{A}^{\prime}, \mathcal{L}^{\prime}, a^{\prime}\right)$ be another $\mathcal{R}$-twisting datum on $X$. Consider the semisimplicial complex $X^{[\bullet]} \times{ }_{X} X^{\prime[\bullet]} \stackrel{p}{\rightarrow} X$, and denote by $\pi^{[\bullet]}: X^{[\bullet]} \times_{X} X^{\prime[\bullet]} \rightarrow$ $X^{[\bullet]}$ and $\pi^{\prime[\bullet]}: X^{[\bullet]} \times_{X} X^{\prime[\bullet]} \rightarrow X^{\prime[\bullet]}$ the natural maps. If $\mathcal{F}$ is a sheaf on $X^{[r]}$ and $\mathcal{F}^{\prime}$ is a sheaf on $X^{\prime[r]}$, write for short $\mathcal{F} \otimes_{\mathcal{R}} \mathcal{F}^{\prime}=\left(\pi^{[r]}\right)^{-1} \mathcal{F} \otimes_{\mathcal{R}}\left(\pi^{\prime[r]}\right)^{-1} \mathcal{F}^{\prime}$ on $X^{[r]} \times_{X} X^{\prime[r]}$. The product of $\mathrm{t}$ and $\mathrm{t}^{\prime}$ is the $\mathcal{R}$-twisting datum on $X$

$$
\mathrm{t} \otimes_{\mathcal{R}} \mathrm{t}^{\prime}=\left(X^{[\bullet]} \times_{X} X^{\prime[\bullet]} \stackrel{p}{\rightarrow} X, \mathcal{A} \otimes_{\mathcal{R}} \mathcal{A}^{\prime}, \mathcal{L} \otimes_{\mathcal{R}} \mathcal{L}^{\prime}, a \otimes_{\mathcal{R}} a^{\prime}\right) .
$$

Let $f: Y \rightarrow X$ be a continuous map of topological spaces. Consider the semisimplicial complex $Y \times_{X} X^{[\bullet]} \stackrel{r}{\rightarrow} Y$, and denote by $f^{[\bullet]}: Y \times_{X} X^{[\bullet]} \rightarrow X^{[\bullet]}$ the natural maps. The pull-back of t by $f$ is the $f^{-1} \mathcal{R}$-twisting datum on $Y$

$$
f^{-1} \mathbf{t}=\left(Y \times_{X} X^{[\bullet]} \stackrel{r}{\rightarrow} Y,\left(f^{[0]}\right)^{-1} \mathcal{A},\left(f^{[1]}\right)^{-1} \mathcal{L},\left(f^{[2]}\right)^{-1} a\right) .
$$

One checks that, for $\mathrm{t}$ and $\mathrm{t}^{\prime}$ satisfying (3.8), there are two $\mathcal{R}$-equivalences and one $f^{-1} \mathcal{R}$-equivalence

$$
\begin{aligned}
\mathfrak{M o d}(\mathrm{t})^{\circledast-1} & \approx \mathfrak{M o d}\left(\mathrm{t}^{\mathrm{op}}\right), \\
\mathfrak{M o d}(\mathrm{t}) \circledast_{\mathcal{R}} \mathfrak{M o d}\left(\mathrm{t}^{\prime}\right) & \approx \mathfrak{M o d}\left(\mathrm{t} \otimes_{\mathcal{R}} \mathrm{t}^{\prime}\right), \\
f^{\circledast} \mathfrak{M o d}(\mathrm{t}) & \approx \mathfrak{M o d}\left(f^{-1} \mathrm{t}\right) .
\end{aligned}
$$


Recall that a topological manifold $X$ is a paracompact Hausdorff topological space locally homeomorphic to $\mathbb{R}^{n}$. In particular, $X$ is locally compact. In the context of twisting data, the sheaf theoretical operations of Proposition 2.17 are easily described under the assumption that $f: Y \rightarrow X$ is a morphism of topological manifolds, and $X^{[\bullet]} \stackrel{q}{\rightarrow} X$ and $X^{\prime[\bullet]} \stackrel{q^{\prime}}{\rightarrow} X$ are semisimplicial complexes of topological manifolds with submersive maps. (Note that this last requirement is automatically fulfilled for twisting data as in (3.2).)

For example, let us describe the direct image functor $f_{*}$. With the same notations as in (3.12), consider the Cartesian squares

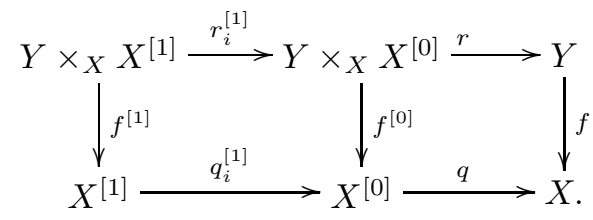

If $(\mathcal{G}, n)$ is an object of $\operatorname{Mod}\left(f^{-1} \mathrm{t}\right)$, then $f_{*}(\mathcal{G}, n)=\left(f_{*}^{[0]} \mathcal{G}, f_{*} n\right)$, where $f_{*} n$ is the composite

$$
\begin{aligned}
\mathcal{L} \otimes_{\mathcal{A}_{1}}\left(q_{1}^{[1]}\right)^{-1} f_{*}^{[0]} \mathcal{G} & \simeq \mathcal{L} \otimes_{\mathcal{A}_{1}} f_{*}^{[1]}\left(r_{1}^{[1]}\right)^{-1} \mathcal{G} \\
& \simeq f_{*}^{[1]}\left(\left(f^{[1]}\right)^{-1} \mathcal{L} \otimes_{\left(f^{[1]}\right)^{-1} \mathcal{A}_{1}}\left(r_{1}^{[1]}\right)^{-1} \mathcal{G}\right) \\
& \stackrel{\sim}{\vec{n}} f_{*}^{[1]}\left(r_{0}^{[1]}\right)^{-1} \mathcal{G} \\
& \simeq\left(q_{0}^{[1]}\right)^{-1} f_{*}^{[0]} \mathcal{G} .
\end{aligned}
$$

Here, the first and last isomorphisms hold because the maps $q_{i}^{[1]}$,s are submersive (and hence so are the $r_{i}^{[1]}$ 's), while the second isomorphism is due the fact that $\mathcal{L}$ is a flat $\mathcal{A}_{1}$-module locally of finite presentation, and hence locally a direct summand of a free $\mathcal{A}_{1}$-module of finite rank.

\subsection{Complex powers of line bundles.}

Let us discuss the example of twisting data attached to line bundles.

Let $X$ be a complex analytic manifold, and denote by $\mathcal{O}_{X}$ its structural sheaf. Let $\pi: F \rightarrow X$ be a line bundle, let $q: Y=F \backslash X \rightarrow X$ be the associated principal $\mathbb{C}^{\times}$-bundle obtained by removing the zero-section, and denote by $\mathcal{F}$ the sheaf of sections of $\pi$.

As in Example 3.4 (a2), consider the semisimplicial complex where $X^{[r]}$ is the $(r+1)$-fold fibered product of $Y$. For $\lambda \in \mathbb{C}$, one has a local system on $X^{[1]}$

$$
\mathcal{L}^{\lambda}=p^{-1} \mathbb{C} t^{\lambda}
$$


where $p: X^{[1]} \rightarrow \mathbb{C}^{\times}$is the map $(x, y) \mapsto x / y$, and $\mathbb{C} t^{\lambda} \subset \mathcal{O}_{\mathbb{C}^{\times}}$is the local system on $\mathbb{C}^{\times}$generated by $t^{\lambda}$. This defines a $\mathbb{C}_{X}$-twisting datum

$$
\mathrm{t}^{\lambda}=\left(X^{[\bullet]} \stackrel{q}{\rightarrow} X, \mathbb{C}_{Y}, \mathcal{L}^{\lambda}, a\right),
$$

where $a$ is given by $\left(c(x / y)^{\lambda}, d(y / z)^{\lambda}\right) \mapsto c d(x / z)^{\lambda}$.

Denote by $\mathcal{O}_{Y}(\lambda)$ the subsheaf of $\mathcal{O}_{Y}$ of $\lambda$-homogeneous functions, i.e. solutions of $e u-\lambda$, where $e u$ is the infinitesimal generator of the action of $\mathbb{C}^{\times}$on the fibers of $q$. It is a $q^{-1} \mathcal{O}_{X}$-module locally constant along the fibers of $q$, and there is a natural isomorphism $m: \mathcal{L}^{\lambda} \otimes\left(q_{1}^{[1]}\right)^{-1} \mathcal{O}_{Y}(\lambda) \rightarrow\left(q_{0}^{[1]}\right)^{-1} \mathcal{O}_{Y}(\lambda)$ on $X^{[1]}$ given by $\left(c(x / y)^{\lambda}, \varphi(y)\right) \mapsto c \varphi(x)$. This gives an object

$$
\mathcal{F}^{-\lambda}=\left(\mathcal{O}_{Y}(\lambda), m\right) \in \operatorname{Mod}\left(\mathcal{O}_{X} ; \mathrm{t}^{\lambda}\right) .
$$

The choice of sign is due to the fact that there is an isomorphism

$$
\mathcal{F} \stackrel{\sim}{\rightarrow} q_{*} \mathcal{O}_{Y}(-1)
$$

given by $\varphi \mapsto(x \mapsto \varphi(q(x)) / x)$, with inverse $\psi \mapsto(x \mapsto \psi(x) x)$.

\section{Examples and applications}

Giraud 14] uses gerbes to define the second cohomology of a sheaf of not necessarily commutative groups $\mathcal{G},{ }^{15}$ and if $\mathcal{G}$ is abelian this provides a geometric description of the usual cohomology group $H^{2}(X ; \mathcal{G})$. We consider here the case of a sheaf of commutative local rings $\mathcal{R}$, and recall how $\mathcal{R}$-equivalence classes of stacks of twisted $\mathcal{R}$-modules are in one-to-one correspondence with $H^{2}\left(X ; \mathcal{R}^{\times}\right)$. We also discuss the examples of stacks of twisted modules associated with inner forms of an $\mathcal{R}$-algebra, considering in particular the case of Azumaya algebras and TDO-rings. As an application, we state a twisted version of an adjunction formula for sheaves and $D$-modules in the context of Radon-type integral transforms.

\subsection{Twisted modules over commutative local rings.}

Let $\mathcal{R}$ be a sheaf of commutative rings. With the terminology of Definition 3.2 an $\mathcal{R}$-module is called invertible if it is invertible as $\mathcal{R} \otimes_{\mathcal{R}} \mathcal{R}^{\mathrm{op}}$-module. Denote by $\operatorname{Pic}(\mathcal{R})$ the set of isomorphism classes of invertible $\mathcal{R}$-modules, endowed with the abelian group law given by tensor product over $\mathcal{R}$. This is called the Picard group of $\mathcal{R}$.

Proposition 4.1. Let $\mathfrak{M}$ be a stack of twisted $\mathcal{R}$-modules. The group of isomorphism classes of $\mathcal{R}$-equivalences of $\mathfrak{M}$ to itself is isomorphic to $\operatorname{Pic}(\mathcal{R})$.

\footnotetext{
${ }^{15}$ We will discuss in $[9]$ the linear analogue, where $\mathcal{G}$ is replaced by a not necessarily commutative $\mathcal{R}$-algebra
} 
Proof. To an invertible $\mathcal{R}$-module $\mathcal{L}$, one associates the $\mathcal{R}$-functor $\varphi=\mathcal{L} \otimes_{\mathcal{R}}(\cdot)$. To an $\mathcal{R}$-equivalence $\varphi$ of $\mathfrak{M}$ to itself, one associates the invertible $\mathcal{R}$-module $\mathcal{L}=\mathcal{H} o m\left(\operatorname{id}_{\mathfrak{M}}, \varphi\right)$.

Let $\mathfrak{M}$ be a stack of twisted $\mathcal{R}$-modules, and denote by $[\mathfrak{M}]$ its $\mathcal{R}$-equivalence class. The multiplication $[\mathfrak{M}]\left[\mathfrak{M}^{\prime}\right]=\left[\mathfrak{M} \circledast_{\mathcal{R}} \mathfrak{M}^{\prime}\right]$ is well defined, with identity $[\mathfrak{M o d}(\mathcal{R})]$ and inverse $[\mathfrak{M}]^{-1}=\left[\mathfrak{M}^{\circledast-1}\right]$. Let us denote by $\operatorname{Tw}(\mathcal{R})$ the set of $\mathcal{R}$ equivalence classes of stacks of twisted $\mathcal{R}$-modules endowed with this abelian group structure.

Definition 4.2. Let us say that $\mathcal{R}$ is Picard good if invertible $\mathcal{R}$-modules are locally isomorphic to $\mathcal{R}$ itself.

Recall that a sheaf of commutative rings $\mathcal{R}$ is called local if for any $U \subset X$ and any $r \in \mathcal{R}(U)$ there exists an open covering $\left\{V_{i}\right\}_{i \in I}$ of $U$ such that for any $i \in I$ either $\mathcal{R} / \mathcal{R} r=0$ or $\mathcal{R} / \mathcal{R}(1-r)=0$ on $V_{i}$. Sheaves of commutative local rings are examples of Picard good rings.

In the rest of this section we assume that $\mathcal{R}$ is Picard good. Denote by $\mathcal{R}^{\times}$the multiplicative group of invertible elements in $\mathcal{R}$.

Proposition 4.3. $\quad$ (i) There is a group isomorphism $\operatorname{Pic}(\mathcal{R}) \simeq H^{1}\left(X ; \mathcal{R}^{\times}\right)$.

(ii) There is a group isomorphism $\mathrm{Tw}(\mathcal{R}) \simeq H^{2}\left(X ; \mathcal{R}^{\times}\right)$.

Part (i) easily follows from the definition of Picard good. Part (ii) of the above proposition is proved as the analogue result for gerbes discussed e.g. in [5] §2.7]. Recall that $H^{2}\left(X ; \mathcal{R}^{\times}\right)$is calculated using hypercoverings, and coincides with Cech cohomology if $X$ is Hausdorff paracompact.

Definition 4.4. Let $\mathfrak{M}$ be a stack of twisted $\mathcal{R}$-modules. We say that $\mathcal{F} \in \mathfrak{M}(X)$ is a locally free twisted $\mathcal{R}$-module of finite rank if there exists a covering $\left\{U_{i}\right\}_{i \in I}$ of $X$, and $\left.\mathcal{R}\right|_{U_{i}}$-equivalences $\varphi_{i}:\left.\mathfrak{M}\right|_{U_{i}} \rightarrow \mathfrak{M o d}\left(\left.\mathcal{R}\right|_{U_{i}}\right)$, such that $\varphi_{i}\left(\left.\mathcal{F}\right|_{U_{i}}\right)$ is a locally free $\left.\mathcal{R}\right|_{U_{i}}$-module of finite rank. More generally, for an $\mathcal{R}$-algebra $\mathcal{A}$ we will speak of locally free $\mathcal{R}$-twisted $\mathcal{A}$-modules of finite $\operatorname{rank}$ in $\operatorname{Mod}(\mathcal{A} ; \mathfrak{M})$.

Note that if $\mathcal{F}$ is a locally free twisted $\mathcal{R}$-module of finite rank, then for any $\left.\mathcal{R}\right|_{U}$-equivalence $\varphi:\left.\mathfrak{M}\right|_{U} \rightarrow \mathfrak{M o d}\left(\left.\mathcal{R}\right|_{U}\right), \varphi(\mathcal{F})$ is a locally free $\mathcal{R}$-module of finite rank. Note also that the rank of $\mathcal{F}$ is a well defined locally constant function.

Proposition 4.5. Let $\mathfrak{M}$ be a stack of twisted $\mathcal{R}$-modules.

(i) $\mathfrak{M}$ is $\mathcal{R}$-equivalent to $\mathfrak{M o d}(\mathcal{R})$ if and only if $\mathfrak{M}(X)$ has a locally free twisted $\mathcal{R}$-module of rank 1 .

(ii) More generally, $\mathfrak{M}$ is $\mathcal{R}$-equivalent to another stack of twisted $\mathcal{R}$-modules $\mathfrak{N}$ if and only if $\mathfrak{M}^{\circledast-1} \circledast_{\mathcal{R}} \mathfrak{N}(X)$ has a locally free twisted $\mathcal{R}$-module of rank 1 .

(iii) If $\mathfrak{M}(X)$ has a locally free twisted $\mathcal{R}$-module of rank $n$, then $n$-fold product $\mathfrak{M}^{\circledast n}=\mathfrak{M}_{\mathcal{R}} \cdots \circledast_{\mathcal{R}} \mathfrak{M}$ is $\mathcal{R}$-equivalent to $\mathfrak{M o d}(\mathcal{R})$. 
Proof. To a locally free twisted $\mathcal{R}$-module $\mathcal{L}$ of rank 1 in $\mathfrak{M}(X)$ one associates the $\mathcal{R}$-equivalence $\mathcal{L} \otimes_{\mathcal{R}}(\cdot): \mathfrak{M o d}(\mathcal{R}) \rightarrow \mathfrak{M}$. To an $\mathcal{R}$-equivalence $\varphi: \mathfrak{M o d}(\mathcal{R}) \rightarrow \mathfrak{M}$, one associates the locally free twisted $\mathcal{R}$-module of rank one $\varphi(\mathcal{R})$. This proves (i). (ii) follows from (i). As for (iii), let $\mathcal{F} \in \mathfrak{M}(X)$ be a locally free twisted $\mathcal{R}$-module of rank $n$. Then $\operatorname{det} \mathcal{F}$ is a locally free twisted $\mathcal{R}$-module of rank 1 in $\mathfrak{M}^{\circledast n}$.

\subsection{Twisting by inner forms.}

Let $\mathcal{R}$ be a Picard good sheaf of commutative rings, and let $\mathcal{A}$ be an $\mathcal{R}$-algebra. Denote by $\mathcal{A} u t_{\mathcal{R} \text {-alg }}(\mathcal{A})$ the sheaf of groups of automorphisms of $\mathcal{A}$ as an $\mathcal{R}$-algebra, and by $\operatorname{Inn}(\mathcal{A})$ its normal subgroup of inner automorphisms, i.e. the image of the adjunction morphism ad: $\mathcal{A}^{\times} \rightarrow \mathcal{A} u t_{\mathcal{R} \text {-alg }}(\mathcal{A}), a \mapsto\left(b \mapsto a b a^{-1}\right)$.

Definition 4.6. An $\mathcal{R}$-algebra $\mathcal{B}$ is called an inner form of $\mathcal{A}$ if there exist an open covering $\left\{U_{i}\right\}_{i \in I}$ of $X$ and isomorphisms $\theta_{i}:\left.\left.\mathcal{A}\right|_{U_{i}} \stackrel{\sim}{\rightarrow} \mathcal{B}\right|_{U_{i}}$ of $\mathcal{R}$-algebras such that the automorphisms $\theta_{j}^{-1} \circ \theta_{i}$ of $\left.\mathcal{A}\right|_{U_{i j}}$ are inner.

Isomorphism classes of inner forms of $\mathcal{A}$ are classified by $H^{1}(X ; \mathcal{I} n n(\mathcal{A}))$.

Assume that $\mathcal{A}$ is a central $\mathcal{R}$-algebra, i.e. that its center $Z(\mathcal{A})$ is equal to $\mathcal{R}$. (If $\mathcal{A}$ is not central, the following discussion still holds by replacing $\mathcal{R}$ with $Z(\mathcal{A})$.) Then the exact sequence

$$
1 \rightarrow \mathcal{R}^{\times} \rightarrow \mathcal{A}^{\times} \stackrel{\text { ad }}{\longrightarrow} \operatorname{Inn}(\mathcal{A}) \rightarrow 1
$$

induces the exact sequence of pointed sets

$$
H^{1}\left(X ; \mathcal{A}^{\times}\right) \stackrel{\gamma}{\rightarrow} H^{1}(X ; \mathcal{I} n n(\mathcal{A})) \stackrel{\delta}{\rightarrow} H^{2}\left(X ; \mathcal{R}^{\times}\right) .
$$

If $\mathcal{L}$ is a locally free $\mathcal{A}$-module of rank one, then $\gamma([\mathcal{L}])=\left[\mathcal{E}_{n} d_{\mathcal{A}^{\text {op }}}\left(\mathcal{L}^{*}\right)\right]$. If $\mathcal{B}$ is an inner form of $\mathcal{A}$, then $\delta([\mathcal{B}])=\left[\mathfrak{M}_{\mathcal{B}}\right]$, where $\mathfrak{M}_{\mathcal{B}}$ is the stack of twisted $\mathcal{R}$-modules described in the following proposition.

Proposition 4.7. Let $\mathcal{A}$ be a central $\mathcal{R}$-algebra, and $\mathcal{B}$ an inner form of $\mathcal{A}$. Then there exists an $\mathcal{R}$-equivalence $\varphi: \mathfrak{M o d}(\mathcal{B}) \rightarrow \mathfrak{M o d}\left(\mathcal{A} ; \mathfrak{M}_{\mathcal{B}}\right)$, where $\mathfrak{M}_{\mathcal{B}}$ is a stack of twisted $\mathcal{R}$-modules. Moreover, $\varphi \simeq \mathcal{L}_{\mathcal{B}} \otimes_{\mathcal{B}}(\cdot)$ where $\mathcal{L}_{\mathcal{B}}=\varphi(\mathcal{B})$ is a locally free $\mathcal{R}$-twisted $\mathcal{A}$-module of rank one in $\operatorname{Mod}\left(\mathcal{A} \otimes_{\mathcal{R}} \mathcal{B}^{\text {op }} ; \mathfrak{M}_{\mathcal{B}}\right)$, and there is an isomorphism of $\mathcal{R}$-algebras $\mathcal{B} \simeq \mathcal{E} d_{\mathcal{A}^{\text {op }}}\left(\mathcal{L}_{\mathcal{B}}^{* \mathcal{A}}\right)$, where $\mathcal{L}_{\mathcal{B}}^{* \mathcal{A}}=\mathcal{H} m_{\mathcal{A}}\left(\mathcal{L}_{\mathcal{B}}, \mathcal{A}\right) \in$ $\operatorname{Mod}\left(\mathcal{B} \otimes_{\mathcal{R}} \mathcal{A}^{\mathrm{op}} ; \mathfrak{M}_{\mathcal{B}}^{\circledast-1}\right)$.

Proof. Since $\mathcal{B}$ is an inner form of $\mathcal{A}$, there exist an open covering $\left\{U_{i}\right\}_{i \in I}$ of $X$, and isomorphisms $\theta_{i}:\left.\left.\mathcal{A}\right|_{U_{i}} \stackrel{\sim}{\rightarrow} \mathcal{B}\right|_{U_{i}}$ of $\mathcal{R}$-algebras such that $\theta_{j}^{-1} \circ \theta_{i}$ are inner. Let $\varphi_{i}: \mathfrak{M o d}\left(\left.\mathcal{B}\right|_{U_{i}}\right) \rightarrow \mathfrak{M o d}\left(\left.\mathcal{A}\right|_{U_{i}}\right)$ be the induced $\left.\mathcal{R}\right|_{U_{i}}$-equivalences, denote by $\psi_{i}$ a quasi-inverse to $\varphi_{i}$, set $\varphi_{i j}=\left.\left.\varphi_{i}\right|_{U_{i j}} \circ \psi_{j}\right|_{U_{i j}}$, and let $\alpha_{i j k}: \varphi_{i j} \circ \varphi_{j k} \Rightarrow \varphi_{i k}$ be the associated invertible transformations. One checks that $\varphi_{i j} \simeq \mathrm{id}_{\mathfrak{M}_{\mathfrak{o O}}\left(\left.\mathcal{A}\right|_{U_{i j}}\right.}$, so that $\alpha_{i j k} \in \operatorname{End}\left(\operatorname{id}_{\mathfrak{M} \mathfrak{o d}\left(\left.\mathcal{A}\right|_{U_{i j k}}\right)}\right)^{\times} \simeq \Gamma\left(U_{i j k} ; \mathcal{R}^{\times}\right)$. By Proposition 4.3 (ii), this is thus an $\mathcal{R}$-twisting datum defining a stack of twisted $\mathcal{R}$-modules $\mathfrak{M}_{\mathcal{B}}$. The equivalences $\varphi_{i}$ glue together, giving an equivalence $\varphi: \mathfrak{M o d}(\mathcal{B}) \rightarrow \mathfrak{M o d}\left(\mathcal{A} ; \mathfrak{M}_{\mathcal{B}}\right)$. 
The rest of the statement is a twisted version of Morita theorem. $\mathcal{L}_{\mathcal{B}}=\varphi(\mathcal{B})$ is a locally free $\mathcal{R}$-twisted $\mathcal{A}$-module of $\operatorname{rank}$ one in $\operatorname{Mod}\left(\mathcal{A} ; \mathfrak{M}_{\mathcal{B}}\right)$ which inherits a compatible $\mathcal{B}^{\text {op }}$-module structure by that of $\mathcal{B}$ itself, and is such that $\mathcal{B} \simeq$ $\mathcal{E} n d_{\mathcal{A}^{\mathrm{op}}}\left(\mathcal{L}_{\mathcal{B}}^{* \mathcal{A}}\right)$.

\subsection{Azumaya algebras.}

We consider here modules over Azumaya algebras as natural examples of twisted $\mathcal{R}$-modules. Refer to 14,13 for more details. See also 8, 11, where a twisted version of the Fourier-Mukai transform is discussed, and [16, for applications to mathematical physics.

In this section we assume that $\mathcal{R}$ is a sheaf of commutative local rings on $X$.

Definition 4.8. An Azumaya $\mathcal{R}$-algebra ${ }^{16}$ is an $\mathcal{R}$-algebra locally isomorphic to the endomorphism algebra of a locally free $\mathcal{R}$-module of finite rank. If the rank of such modules is constant and equal to $n$, then one says that the Azumaya $\mathcal{R}$-algebra has rank $n^{2}$.

If $\mathcal{F}$ is a locally free $\mathcal{R}$-module of finite rank, then $\mathcal{R}$ and $\mathcal{E} n d_{\mathcal{R}}(\mathcal{F})$ are Morita equivalent. This is a basic example of Morita equivalence, and is proved by noticing that $\mathcal{F}$ itself is an invertible $\mathcal{R} \otimes_{\mathcal{R}} \mathcal{E} n d_{\mathcal{R}}(\mathcal{F})^{\text {op }}$-module (in fact, one has natural isomorphisms $\mathcal{F}^{*} \otimes_{\mathcal{R}} \mathcal{F} \simeq \mathcal{E} n d_{\mathcal{R}}(\mathcal{F})$ and $\mathcal{F} \otimes_{\mathcal{E}_{n d}(\mathcal{F})} \mathcal{F}^{*} \simeq \mathcal{R}$, where $\left.\mathcal{F}^{*}=\mathcal{H o m}_{\mathcal{R}}(\mathcal{F}, \mathcal{R})\right)$. It follows that if $\mathcal{A}$ is an Azumaya $\mathcal{R}$-algebra then $\mathfrak{M o d}(\mathcal{A})$ provides an example of stack of twisted $\mathcal{R}$-modules. Moreover, the Skolem-Noether theorem (see e.g. 14, Lemme V.4.1]) asserts

Proposition 4.9. Any $\mathcal{R}$-algebra automorphism of an Azumaya $\mathcal{R}$-algebra is inner. In particular, Azumaya $\mathcal{R}$-algebras of rank $n^{2}$ are inner forms of the central $\mathcal{R}$-algebra $M_{n}(\mathcal{R})=\mathcal{E} n d_{\mathcal{R}}\left(\mathcal{R}^{n}\right)$.

Set $G L_{n}(\mathcal{R})=M_{n}(\mathcal{R})^{\times}$, and $P G L_{n}(\mathcal{R})=G L_{n}(\mathcal{R}) / \mathcal{R}^{\times}$. Then the set of $\mathcal{R}$ algebra isomorphism classes of Azumaya $\mathcal{R}$-algebras of rank $n^{2}$ is isomorphic to $H^{1}\left(X ; P G L_{n}(\mathcal{R})\right)$.

Proposition 4.10. Let $\mathcal{A}$ be an Azumaya $\mathcal{R}$-algebra of rank $n^{2}$. Then $\mathfrak{M o d}(\mathcal{A}) \approx$ $\mathfrak{M}_{\mathcal{A}}$ is a stack of twisted $\mathcal{R}$-modules, and there exists a locally free twisted $\mathcal{R}$ modules $\mathcal{F}_{\mathcal{A}}$ of rank $n$ in $\mathfrak{M}_{\mathcal{A}}^{\circledast-1}(X) \approx \operatorname{Mod}\left(\mathcal{A}^{\text {op }}\right)$ such that $\mathcal{A} \simeq \mathcal{E}_{n} d_{\mathcal{R}}\left(\mathcal{F}_{\mathcal{A}}\right)$ as $\mathcal{R}$-algebras.

\footnotetext{
${ }^{16}$ The definition that we give here is good for the analytic topology, or for the étale topology. With this definition, if $\mathcal{A}$ is an Azumaya $\mathcal{R}$-algebra, then the morphism of $\mathcal{R}$-algebras

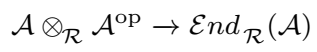

given by $a \otimes b \mapsto(c \mapsto a c b)$ is an isomorphism. For algebraic manifolds with the Zariski topology, it is this property which is sometimes used to define Azumaya $\mathcal{R}$-algebras when $\mathcal{R}$ is the sheaf of rings of regular functions.
} 
Proof. By Proposition 4.7 there exists a stack of twisted $\mathcal{R}$-modules $\mathfrak{M}_{\mathcal{A}}$, and an $\mathcal{R}$-equivalence

$$
\varphi: \mathfrak{M o d}(\mathcal{A}) \rightarrow \mathfrak{M o d}\left(M_{n}(\mathcal{R}) ; \mathfrak{M}_{\mathcal{A}}\right)
$$

The functor $\mathcal{R}^{n} \otimes_{M_{n}(\mathcal{R})}(\cdot)$ gives an $\mathcal{R}$-equivalence $\mathfrak{M o d}\left(M_{n}(\mathcal{R})\right) \rightarrow \mathfrak{M o d}(\mathcal{R})$. By (2.6), this induces an $\mathcal{R}$-equivalence

$$
\psi: \mathfrak{M o d}\left(M_{n}(\mathcal{R}) ; \mathfrak{M}_{\mathcal{A}}\right) \rightarrow \mathfrak{M}_{\mathcal{A}}
$$

Since $\mathcal{A}$ is locally isomorphic to $M_{n}(\mathcal{R}), \psi(\varphi(\mathcal{A}))$ is locally isomorphic to $\mathcal{R}^{n}$. Set $\mathcal{F}_{\mathcal{A}}=\mathcal{H o m}_{\mathcal{R}}(\psi(\varphi(\mathcal{A})), \mathcal{R})$.

With these notations, (4.1) and (4.2) read

$$
1 \rightarrow \mathcal{R}^{\times} \rightarrow G L_{n}(\mathcal{R}) \rightarrow P G L_{n}(\mathcal{R}) \rightarrow 1,
$$

and

$$
H^{1}\left(X ; G L_{n}(\mathcal{R})\right) \stackrel{\gamma_{n}}{\longrightarrow} H^{1}\left(X ; P G L_{n}(\mathcal{R})\right) \stackrel{\delta_{n}}{\longrightarrow} H^{2}\left(X ; \mathcal{R}^{\times}\right),
$$

respectively. If $\mathcal{F}$ is a locally free $\mathcal{R}$-module of $\operatorname{rank} n$, then $\gamma_{n}([\mathcal{F}])=\left[\mathcal{E}_{n} d_{\mathcal{R}}(\mathcal{F})\right]$. If $\mathcal{A}$ is an Azumaya $\mathcal{R}$-algebra of rank $n^{2}$, then $\delta_{n}([\mathcal{A}])=[\mathfrak{M o d}(\mathcal{A})]$.

One says that two Azumaya $\mathcal{R}$-algebras $\mathcal{A}$ and $\mathcal{A}^{\prime}$ are equivalent if there exist two locally free (non twisted) $\mathcal{R}$-modules of finite rank $\mathcal{F}$ and $\mathcal{F}^{\prime}$ such that

$$
\mathcal{A} \otimes_{\mathcal{R}} \mathcal{E} n d_{\mathcal{R}}(\mathcal{F}) \simeq \mathcal{A}^{\prime} \otimes_{\mathcal{R}} \mathcal{E} n d_{\mathcal{R}}\left(\mathcal{F}^{\prime}\right)
$$

Lemma 4.11. Two Azumaya $\mathcal{R}$-algebras are equivalent if and only if they are Morita $\mathcal{R}$-equivalent.

Proof. Since $\mathcal{E} n d_{\mathcal{R}}(\mathcal{F})$ and $\mathcal{R}$ are Morita equivalent, so are $\mathcal{A} \otimes_{\mathcal{R}} \mathcal{E} n d_{\mathcal{R}}(\mathcal{F})$ and $\mathcal{A}$ by (2.6). On the other hand, if $\mathcal{A}$ and $\mathcal{A}^{\prime}$ are Morita equivalent, then there is an $\mathcal{R}$-equivalence $\varphi: \mathfrak{M o d}(\mathcal{R}) \rightarrow \mathfrak{M o d}(\mathcal{A}) \circledast_{\mathcal{R}} \mathfrak{M o d}\left(\mathcal{A}^{\prime}\right)^{\circledast-1} \approx \mathfrak{M o d}\left(\mathcal{A} \otimes_{\mathcal{R}} \mathcal{A}^{\prime \mathrm{op}}\right)$. Hence $\mathcal{A} \otimes_{\mathcal{R}} \mathcal{A}^{\prime \mathrm{op}} \simeq \mathcal{E} n d_{\mathcal{R}}(\mathcal{F})$ for $\mathcal{F}=\varphi(\mathcal{R})$. Tensoring with $\mathcal{A}^{\prime}$ we finally get an isomorphism $\mathcal{A} \otimes_{\mathcal{R}} \mathcal{E} n d_{\mathcal{R}}\left(\mathcal{A}^{\prime}\right) \simeq \mathcal{A}^{\prime} \otimes_{\mathcal{R}} \mathcal{E} n d_{\mathcal{R}}(\mathcal{F})$.

Denote by $[\mathcal{A}]$ the equivalence class of $\mathcal{A}$. The multiplication $[\mathcal{A}]\left[\mathcal{A}^{\prime}\right]=\left[\mathcal{A} \otimes_{\mathcal{R}} \mathcal{A}^{\prime}\right]$ is well defined, with identity $[\mathcal{R}]$ and inverse $[\mathcal{A}]^{-1}=\left[\mathcal{A}^{\text {op }}\right]$. Denote by $\operatorname{Br}(\mathcal{R})$ the set of equivalence classes of Azumaya $\mathcal{R}$-algebras endowed with this abelian group law, which is called Brauer group of $\mathcal{R}$. By the Skolem-Noether theorem one has a group isomorphism

$$
\operatorname{Br}(\mathcal{R}) \simeq \underset{n}{\lim } H^{1}\left(X ; P G L_{n}(\mathcal{R})\right) .
$$

The limit of the maps $\delta_{n}$ in (4.3) gives a group homomorphism

$$
\delta: \operatorname{Br}(\mathcal{R}) \rightarrow \operatorname{Tw}(\mathcal{R})
$$

which is described by $[\mathcal{A}] \mapsto[\mathfrak{M o d}(\mathcal{A})]$. 
Proposition 4.12. The homomorphism $\delta$ is injective, and its image is contained in the torsion part of $\mathrm{Tw}(\mathcal{R})$.

Proof. Let $\mathcal{A}$ be an Azumaya $\mathcal{R}$-algebra of rank $n^{2}$. Let $\mathcal{F}_{\mathcal{A}}$ be the locally free twisted $\mathcal{R}$ module of rank $n$ in $\operatorname{Mod}\left(\mathcal{A}^{\mathrm{op}}\right)$ of Proposition 4.10 If $\delta([\mathcal{A}])=$ $[\mathfrak{M o d}(\mathcal{A})]=0$, then $\mathcal{F}_{\mathcal{A}}$ is not twisted, and $[\mathcal{A}]=\left[\mathcal{E} n d_{\mathcal{R}}\left(\mathcal{F}_{\mathcal{A}}\right)\right]=0$. This proves the injectivity. As for the description of the image, by Proposition 4.5 (iii) one has $n \cdot[\mathfrak{M o d}(\mathcal{A})]=-\left[\mathfrak{M o d}\left(\mathcal{A}^{\mathrm{op}}\right)^{\circledast n}\right]=0$.

\subsection{Twisted differential operators.}

Rings of twisted differential operators (TDO-rings for short) were introduced in a representation theoretical context in 3, 4. Modules over TDO-rings provide another example of twisted modules, and we recall here these facts following the presentation in 18] (see also [27]). Since we deal with complex analytic manifolds, as opposed to algebraic varieties, many arguments are simpler than in loc. cit.

Let $X$ be a complex analytic manifold, and denote by $\mathcal{O}_{X}$ its structural sheaf of holomorphic functions. Recall that an $\mathcal{O}_{X}$-ring is a $\mathbb{C}$-algebra $\mathcal{A}$ endowed with a morphism of $\mathbb{C}$-algebras $\beta: \mathcal{O}_{X} \rightarrow \mathcal{A}$. Morphisms of $\mathcal{O}_{X}$-rings are morphisms of $\mathbb{C}$-algebras compatible with $\beta$.

Denote by $\mathcal{D}_{X}$ the sheaf of differential operators on $X$. Recall that $\mathcal{D}_{X}$ is a simple $\mathcal{O}_{X}$-ring with center $\mathbb{C}_{X}$.

Definition 4.13. A TDO-ring on $X$, short for ring of twisted differential operators, is an $\mathcal{O}_{X}$-ring locally isomorphic to $\mathcal{D}_{X}$ as $\mathcal{O}_{X}$-ring.

A TDO-ring $\mathcal{A}$ has a natural increasing exhaustive filtration defined by induction by $F_{-1} \mathcal{A}=0, F_{m+1} \mathcal{A}=\left\{P \in \mathcal{A}:[P, a] \in F_{m} \mathcal{A} \forall a \in \mathcal{O}_{X}\right\}$, where $[P, Q]=P Q-Q P$ is the commutator. Note that $F_{m+1} \mathcal{A}=F_{1} \mathcal{A} F_{m} \mathcal{A}$ for $m \geq 0$, and that the associated graded algebra $G \mathcal{A}$ is naturally isomorphic to $S_{\mathcal{O}_{X}}\left(\Theta_{X}\right)$, the symmetric algebra of vector fields over $\mathcal{O}_{X}$.

Proposition 4.14. There are group isomorphisms

$$
\mathcal{A} u t_{\mathcal{O}_{X-\text { ring }}}(\mathcal{A}) \simeq d \mathcal{O}_{X} \simeq \operatorname{Inn}(\mathcal{A}) .
$$

In particular, TDO-rings are inner forms of the central $\mathbb{C}$-algebra $\mathcal{D}_{X}$.

Proof. One has $\mathcal{A}^{\times}=\mathcal{O}_{X}^{\times}$. Hence the short exact sequence

$$
1 \rightarrow \mathbb{C}_{X}^{\times} \rightarrow \mathcal{O}_{X}^{\times} \stackrel{d \log }{\longrightarrow} d \mathcal{O}_{X} \rightarrow 0
$$

gives a group isomorphism $\operatorname{Inn}(\mathcal{A}) \simeq d \mathcal{O}_{X}$. This proves the second isomorphism. To prove the first, note that any $\mathcal{O}_{X}$-ring automorphism $\varphi$ of $\mathcal{A}$ preserves the filtration. Let $\omega \in d \mathcal{O}_{X}, P \in F_{1} \mathcal{A}$, and denote by $\sigma_{1}(P) \in \Theta_{X}$ its symbol of order one. Then $P \mapsto P+\left\langle\sigma_{1}(P), \omega\right\rangle$ extends uniquely to an $\mathcal{O}_{X}$-ring automorphism of $\mathcal{A}$. On the other hand, to an $\mathcal{O}_{X}$-ring automorphism $\varphi$ of $\mathcal{A}$ one associates the closed form $\theta \mapsto \varphi(\tilde{\theta})-\tilde{\theta}$, where $\tilde{\theta} \in F_{1}(\mathcal{A})$ is such that $\sigma_{1}(\tilde{\theta})=\theta$. 
Let $\mathcal{F}$ be a locally free $\mathcal{O}_{X}$-module of rank one, and set $\mathcal{F}^{*}=\mathcal{H}^{\circ} m_{\mathcal{O}_{X}}\left(\mathcal{F}, \mathcal{O}_{X}\right)$. Then the basic example of TDO-ring is given by

$$
\mathcal{D}_{\mathcal{F}}=\mathcal{F} \underset{\mathcal{O}}{\otimes} \mathcal{D}_{X} \underset{\mathcal{O}}{\otimes} \mathcal{F}^{*}
$$

where $\left(s \otimes P \otimes s^{*}\right) \cdot\left(t \otimes Q \otimes t^{*}\right)=s \otimes P\left\langle t, s^{*}\right\rangle Q \otimes t^{*}$. Equivalently, $\mathcal{D}_{\mathcal{F}}$ is the sheaf of differential endomorphisms of $\mathcal{F}$, i.e. $\mathbb{C}$-endomorphisms $\varphi$ such that for any $s \in \mathcal{F}$ there exists $P \in \mathcal{D}_{X}$ with $\varphi(a s)=P(a) s$ for any $a \in \mathcal{O}_{X}$. More generally, for $\lambda \in \mathbb{C}$ one has the TDO-ring

$$
\mathcal{D}_{\mathcal{F}^{\lambda}}=\mathcal{F}^{\lambda} \underset{\mathcal{O}}{\otimes} \mathcal{D}_{X} \underset{\mathcal{O}}{\otimes} \mathcal{F}^{-\lambda}
$$

where $\mathcal{F}^{\lambda}$ was described in Section [3.6 By definition, sections of $\mathcal{D}_{\mathcal{F} \lambda}$ are locally of the form $s^{\lambda} \otimes P \otimes s^{-\lambda}$, where $s$ is a nowhere vanishing local section of $\mathcal{F}$, with the gluing condition $s^{\lambda} \otimes P \otimes s^{-\lambda}=t^{\lambda} \otimes Q \otimes t^{-\lambda}$ if and only if $Q=(s / t)^{\lambda} P(s / t)^{-\lambda}$. This is independent from the choice of a branch for the ramified function $s^{\lambda}$.

Proposition 4.15. Let $\mathcal{A}$ be a TDO-ring. Then there exists a stack of twisted $\mathbb{C}_{X}$-modules $\mathfrak{M}_{\mathcal{A}}$ such that $\mathfrak{M o d}(\mathcal{A})$ is $\mathbb{C}$-equivalent to $\mathfrak{M}_{0} \mathfrak{d}\left(\mathcal{D}_{X} ; \mathfrak{M}_{\mathcal{A}}\right)$. Moreover, in $\operatorname{Mod}\left(\mathcal{O}_{X} ; \mathfrak{M}_{\mathcal{A}}^{\circledast-1}\right)$ there exists a locally free $\mathbb{C}_{X}$-twisted $\mathcal{O}_{X}$-module of rank one $\mathcal{O}_{\mathcal{A}}$, such that $\mathcal{A} \simeq \mathcal{D}_{\mathcal{O}_{\mathcal{A}}}$ as $\mathcal{O}_{X}$-rings.

Proof. By Proposition 4.7 there exists a stack of twisted $\mathbb{C}_{X}$-modules $\mathfrak{M}_{\mathcal{A}}$, and a $\mathbb{C}$-equivalence

$$
\varphi: \mathfrak{M o d}(\mathcal{A}) \rightarrow \mathfrak{M o d}\left(\mathcal{D}_{X} ; \mathfrak{M}_{\mathcal{A}}\right)
$$

Since $\mathcal{D}_{X}^{\times}=\mathcal{O}_{X}^{\times}$, any locally free $\mathbb{C}_{X}$-twisted $\mathcal{D}_{X}$-module of rank one is isomorphic to $\mathcal{D}_{X} \otimes_{\mathcal{O}_{X}} \mathcal{F}$ for a locally free $\mathbb{C}_{X}$-twisted $\mathcal{O}_{X}$-module of rank one $\mathcal{F}$. In particular, $\varphi(\mathcal{A}) \simeq \mathcal{D}_{X} \otimes_{\mathcal{O}_{X}} \mathcal{F}_{\mathcal{A}}$ for some $\mathcal{F}_{\mathcal{A}} \in \operatorname{Mod}\left(\mathcal{O}_{X} ; \mathfrak{M}_{\mathcal{A}}\right)$, and we set $\mathcal{O}_{\mathcal{A}}=\operatorname{Hom}_{\mathcal{O}_{X}}\left(\mathcal{F}_{\mathcal{A}}, \mathcal{O}_{X}\right)$.

Conjecture 4.16. Any stack of $\mathbb{C}_{X}$-twisted $\mathcal{D}_{X}$-modules is $\mathbb{C}$-equivalent to a stack of the form $\mathfrak{M o d}\left(\mathcal{D}_{X} ; \mathfrak{M}\right)$ for some stack of twisted $\mathbb{C}_{X}$-modules $\mathfrak{M}$.

Denote by $\Omega_{X}$ the sheaf of differential forms of top degree, and recall that there is a natural isomorphism of $\mathcal{O}_{X}$-rings $\mathcal{D}_{X}^{\text {op }} \simeq \mathcal{D}_{\Omega_{X}}$. To the TDO-rings $\mathcal{A}$ and $\mathcal{A}^{\prime}$ one associates the TDO-rings

$$
\mathcal{A} \sharp \mathcal{A}^{\prime}=\mathcal{E} n d_{\mathcal{A} \otimes \mathcal{A}^{\prime}}\left(\mathcal{A} \underset{\mathcal{O}}{\otimes} \mathcal{A}^{\prime}\right), \quad \mathcal{A}^{\sharp-1}=\Omega_{X}^{*} \underset{\mathcal{O}}{\otimes} \mathcal{A}^{\mathrm{op}}{\underset{\mathcal{O}}{\otimes}}_{\Omega_{X}},
$$

where in the right-hand-side of the first equation $\mathcal{A}$ and $\mathcal{A}^{\prime}$ are regarded as $\mathcal{O}_{X^{-}}$ modules by left multiplication and $\mathcal{A} \underset{\mathcal{O}}{\otimes} \mathcal{A}^{\prime}$ is regarded as an $\mathcal{A} \underset{\mathbb{C}}{\otimes} \mathcal{A}^{\prime}$-module by right multiplication. Note that if $\mathcal{F}$ and $\mathcal{F}^{\prime}$ are locally free twisted $\mathcal{O}_{X}$-modules of rank one, then

$$
\begin{aligned}
\mathcal{D}_{\mathcal{F}}^{\sharp-1} & \simeq \mathcal{D}_{\mathcal{F} *}, \\
\mathcal{D}_{\mathcal{F}} \sharp \mathcal{D}_{\mathcal{F}^{\prime}} & \simeq \mathcal{D}_{\mathcal{F} \otimes_{\mathcal{O}} \mathcal{F}^{\prime}},
\end{aligned}
$$


where $\mathcal{F}^{*}=\mathcal{H o m}_{\mathcal{O}_{X}}\left(\mathcal{F}, \mathcal{O}_{X}\right)$.

Let us denote by $[\mathcal{A}]$ the $\mathcal{O}_{X}$-ring isomorphism class of $\mathcal{A}$. The multiplication $[\mathcal{A}]\left[\mathcal{A}^{\prime}\right]=\left[\mathcal{A} \sharp \mathcal{A}^{\prime}\right]$ is well defined, with identity $\left[\mathcal{D}_{X}\right]$ and inverse $[\mathcal{A}]^{-1}=\left[\mathcal{A}^{\sharp-1}\right]$. Let us denote by $\operatorname{TDO}\left(\mathcal{O}_{X}\right)$ the set of $\mathcal{O}_{X}$-ring isomorphism classes of TDO-rings, endowed with this abelian group law. As a corollary of Proposition 4.14 we get

Proposition 4.17. There is a group isomorphism $\operatorname{TDO}\left(\mathcal{O}_{X}\right) \simeq H^{1}\left(X ; d \mathcal{O}_{X}\right)$.

For inner forms of $\mathcal{D}_{X}$, the short exact sequence (4.1) reads

$$
1 \rightarrow \mathbb{C}_{X}^{\times} \rightarrow \mathcal{O}_{X}^{\times} \stackrel{d \log }{\longrightarrow} d \mathcal{O}_{X} \rightarrow 0 .
$$

It induces the long exact cohomology sequence

$$
H^{1}\left(X ; \mathbb{C}_{X}^{\times}\right) \rightarrow H^{1}\left(X ; \mathcal{O}_{X}^{\times}\right) \rightarrow H^{1}\left(X ; d \mathcal{O}_{X}\right) \rightarrow H^{2}\left(X ; \mathbb{C}_{X}^{\times}\right) \rightarrow H^{2}\left(X ; \mathcal{O}_{X}^{\times}\right),
$$

which may be written as the exact sequence of groups

$$
\operatorname{Pic}\left(\mathbb{C}_{X}\right) \rightarrow \operatorname{Pic}\left(\mathcal{O}_{X}\right) \stackrel{\gamma}{\rightarrow} \operatorname{TDO}\left(\mathcal{O}_{X}\right) \stackrel{\delta}{\rightarrow} \operatorname{Tw}\left(\mathbb{C}_{X}\right) \rightarrow \operatorname{Tw}\left(\mathcal{O}_{X}\right),
$$

where $\gamma([\mathcal{F}])=\left[\mathcal{D}_{\mathcal{F}}\right]$ and $\delta([\mathcal{A}])=\left[\mathfrak{M}_{\mathcal{A}}\right]$. Note that [4.5) implies the relations $\left[\mathcal{A}^{\mathrm{op}}\right]=\gamma\left(\left[\Omega_{X}\right]\right)-[\mathcal{A}]$, and $\left[\mathfrak{M}_{\mathcal{A}}\right]=-\left[\mathfrak{M}_{\mathcal{A}^{\mathrm{op}}}\right]$. Note also that the complex span of the image of $\gamma$ is described by $\lambda \cdot \gamma([\mathcal{F}])=\left[\mathcal{D}_{\mathcal{F}^{\lambda}}\right]$, for $\lambda \in \mathbb{C}$.

Example 4.18. Let $X=\mathbb{P}$ be a complex finite dimensional projective space. Then the above long exact sequence reads $0 \rightarrow \mathbb{Z} \rightarrow \mathbb{C} \rightarrow \mathbb{C} / \mathbb{Z} \rightarrow 0$. Denote by $\mathcal{O}_{\mathbb{P}}(-1)$ the tautological line bundle, and for $\lambda \in \mathbb{C}$ set $\mathcal{O}_{\mathbb{P}}(\lambda)=\left(\mathcal{O}_{\mathbb{P}}(-1)\right)^{-\lambda}$. Then

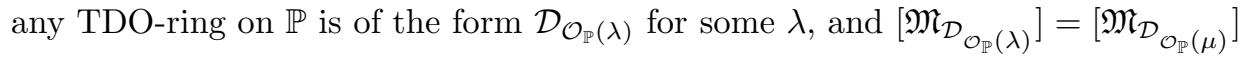
if and only if $\lambda-\mu \in \mathbb{Z}$. In this case, an equivalence $\mathfrak{M o d}\left(\mathcal{D}_{\mathcal{O}_{\mathbb{P}}(\lambda)}\right) \rightarrow \mathfrak{M o d}\left(\mathcal{D}_{\mathcal{O}_{\mathbb{P}}(\mu)}\right)$ is given by $\mathcal{O}_{\mathbb{P}}(\mu-\lambda) \otimes_{\mathcal{O}_{X}}(\cdot)$.

\subsection{Twisted $\mathcal{D}$-module operations.}

We recall here the twisted analogue of $D$-module operations, following [18, 27. (We do not recall here the classical formalism of operations for $D$-modules, referring instead to 21, 19.)

Besides the internal operations for TDO-rings recalled in (4.5), there is an external operation defined as follows. Let $f: Y \rightarrow X$ be a morphism of complex analytic manifolds. To a TDO-ring $\mathcal{A}$ on $X$ one associates the TDO-ring on $Y$

$$
f^{\sharp} \mathcal{A}=\mathcal{E} n d_{f^{-1} \mathcal{A}}\left(\mathcal{O}_{Y} \otimes_{f^{-1} \mathcal{O}_{X}} f^{-1} \mathcal{A}\right),
$$

where $\mathcal{O}_{Y} \otimes_{f^{-1} \mathcal{O}_{X}} f^{-1} \mathcal{A}$ is regarded as a right $f^{-1} \mathcal{A}$-module. One has

$$
\begin{aligned}
f^{\sharp}\left(\mathcal{A}^{\sharp-1}\right) & \simeq\left(f^{\sharp} \mathcal{A}\right)^{\sharp-1}, \\
f^{\sharp}\left(\mathcal{A} \sharp \mathcal{A}^{\prime}\right) & \simeq f^{\sharp} \mathcal{A} \sharp f^{\sharp} \mathcal{A}^{\prime} .
\end{aligned}
$$


Moreover, if $\mathcal{F}$ is a locally free twisted $\mathcal{O}_{X}$-module of rank one, then

$$
f^{\sharp} \mathcal{D}_{\mathcal{F}} \simeq \mathcal{D}_{f * \mathcal{F}},
$$

where $f^{*} \mathcal{F}=\mathcal{O}_{Y} \otimes_{f-1} \mathcal{O}_{X} f^{-1} \mathcal{F}$.

Let $f: Y \rightarrow X$ be a morphism of complex analytic manifolds, and $\mathcal{A}$ a TDOring on $X$. Consider the transfer modules

$$
\begin{array}{ll}
\mathcal{A}_{Y \rightarrow X}=\mathcal{O}_{Y} \otimes_{f^{-1} \mathcal{O}_{X}} f^{-1} \mathcal{A}, & \text { an } f^{\sharp} \mathcal{A} \otimes_{\mathbb{C}_{Y}}\left(f^{-1} \mathcal{A}\right)^{\mathrm{op}} \text {-module, } \\
\mathcal{A}_{X \leftarrow Y}=f^{-1} \mathcal{A} \otimes_{f^{-1} \mathcal{O}_{X}} \Omega_{f}, & \text { an } f^{-1} \mathcal{A} \otimes_{\mathbb{C}_{Y}}\left(f^{\sharp} \mathcal{A}\right)^{\mathrm{op}} \text {-module, }
\end{array}
$$

where $\Omega_{f}=\Omega_{Y} \underset{\mathcal{O}}{\otimes} f^{*} \Omega_{X}^{*}, \Omega_{X}^{*}$ denoting the dual $\mathcal{H o m} \mathcal{O}_{X}\left(\Omega_{X}, \mathcal{O}_{X}\right)$ of $\Omega_{X}$. Note that if $\mathcal{F}$ is a locally free twisted $\mathcal{O}_{X}$-module of rank one, then

$$
\begin{aligned}
& \left(\mathcal{D}_{\mathcal{F}}\right)_{Y \rightarrow X} \simeq f^{*} \mathcal{F} \otimes_{\mathcal{O}_{Y}} \mathcal{D}_{Y \rightarrow X} \otimes_{f^{-1} \mathcal{O}_{X}} f^{-1} \mathcal{F}^{*}, \\
& \left(\mathcal{D}_{\mathcal{F}}\right)_{X \leftarrow Y} \simeq f^{-1} \mathcal{F} \otimes_{f^{-1} \mathcal{O}_{X}} \mathcal{D}_{X \leftarrow Y} \otimes_{\mathcal{O}_{Y}} f^{*} \mathcal{F}^{*},
\end{aligned}
$$

where $\mathcal{D}_{Y \rightarrow X}$ and $\mathcal{D}_{X \leftarrow Y}$ are the classical transfer bimodules.

Let $\mathfrak{M}$ and $\mathfrak{M}^{\prime}$ be two stacks of twisted $\mathbb{C}_{X}$-modules, $\mathcal{A}$ and $\mathcal{A}^{\prime}$ two TDOrings on $X$. Denote by $\mathrm{D}^{\mathrm{b}}(\mathcal{A} ; \mathfrak{M})$ the bounded derived category of $\operatorname{Mod}(\mathcal{A} ; \mathfrak{M})=$ $\mathfrak{M o d}(\mathcal{A} ; \mathfrak{M})(X)$. The usual operations for $D$-modules extend to the twisted case, yielding the functors

$$
\begin{aligned}
& \mathbb{D}: \mathrm{D}^{\mathrm{b}}(\mathcal{A} ; \mathfrak{M}) \times \mathrm{D}^{\mathrm{b}}\left(\mathcal{A}^{\prime} ; \mathfrak{M}^{\prime}\right) \rightarrow \mathrm{D}^{\mathrm{b}}\left(\mathcal{A} \sharp \mathcal{A}^{\prime} ; \mathfrak{M} \circledast \circledast_{\mathbb{C}} \mathfrak{M}^{\prime}\right), \\
& \mathbb{D} f^{*}: \mathrm{D}^{\mathrm{b}}(\mathcal{A} ; \mathfrak{M}) \rightarrow \mathrm{D}^{\mathrm{b}}\left(f^{\sharp} \mathcal{A} ; f^{\circledast} \mathfrak{M}\right), \\
& \mathbb{D} f_{*}: \mathrm{D}^{\mathrm{b}}\left(f^{\sharp} \mathcal{A} ; f^{\circledast} \mathfrak{M}\right) \rightarrow \mathrm{D}^{\mathrm{b}}(\mathcal{A} ; \mathfrak{M}),
\end{aligned}
$$

defined by $\mathcal{M} \stackrel{\mathbb{D}}{\otimes} \mathcal{M}^{\prime}=\mathcal{M} \stackrel{L}{\otimes} \mathcal{M}^{\prime}, \mathbb{D} f^{*} \mathcal{M}=\mathcal{A}_{Y \rightarrow X} \otimes_{f^{-1} \mathcal{A}}^{L} f^{-1} \mathcal{M}$, and $\mathbb{D} f_{*} \mathcal{N}=$ $R f_{*}\left(\mathcal{A}_{X \leftarrow Y} \otimes_{f^{\sharp} \mathcal{A}}^{L} \mathcal{N}\right)$. The usual formulas, like base-change or projection formula, hold. Moreover, all local notions like those of coherent module, of characteristic variety, or of regular holonomic module, still make sense.

We will also consider the functor

$$
R \mathcal{H o m}{ }_{\mathcal{A}}: \mathrm{D}^{\mathrm{b}}\left(\mathcal{A} ; \mathfrak{M}^{\circledast-1}\right)^{\mathrm{op}} \times \mathrm{D}^{\mathrm{b}}\left(\mathcal{A} ; \mathfrak{M}^{\prime}\right) \rightarrow \mathrm{D}^{\mathrm{b}}\left(\mathfrak{M} \circledast_{\mathbb{C}} \mathfrak{M}^{\prime}\right) .
$$

\subsection{Twisted adjunction formula.}

An adjunction formula for sheaves and $D$-modules in the context of Radon-type integral transforms was established in [10. We briefly explain here how such formula generalizes to the twisted case. Note that a twisted adjunction formula for Poisson-type integral transforms was established in [26], where the group action and the topology of functional spaces are also taken into account.

Let $X$ and $Y$ be complex analytic manifolds, $\mathfrak{M}$ a stack of twisted $\mathbb{C}_{X}$-modules, $\mathfrak{N}$ a stack of twisted $\mathbb{C}_{Y}$-modules, $\mathcal{A}$ a TDO-ring on $X$, and $\mathcal{B}$ a TDO-ring on $Y$. We will use the notations $\mathfrak{M}_{\mathcal{A}}$ and $\mathcal{O}_{\mathcal{A}}$ from Proposition 4.15. Consider the natural 
projections

$$
X \stackrel{\pi_{1}}{\longleftarrow} X \times Y \stackrel{\pi_{2}}{\longrightarrow} Y
$$

and set

$$
\begin{aligned}
\mathfrak{M} * \mathfrak{N} & =\pi_{1}^{\circledast} \mathfrak{M} \circledast_{\mathbb{C}} \pi_{2}^{\circledast} \mathfrak{N}, \\
\mathcal{A} \boxplus \mathcal{B} & =\pi_{1}^{\sharp} \mathcal{A} \sharp \pi_{2}^{\sharp} \mathcal{B} .
\end{aligned}
$$

To $K \in \mathrm{D}^{\mathrm{b}}\left(\mathfrak{M} \circledast \mathfrak{N}^{\circledast-1}\right)$ one associates the functor

$$
K \circ(\cdot): \mathrm{D}^{\mathrm{b}}(\mathfrak{N}) \rightarrow \mathrm{D}^{\mathrm{b}}(\mathfrak{M}), \quad G \mapsto R \pi_{1 !}\left(K \otimes \pi_{2}^{-1} G\right) .
$$

To $\mathcal{K} \in \mathrm{D}^{\mathrm{b}}\left(\mathcal{A}^{\sharp-1} \boxplus \mathcal{B} ; \mathfrak{M}^{\circledast-1}\right.$ 困 $\left.\mathfrak{N}\right)$ one associates the functor

$$
(\cdot) \stackrel{\mathbb{D}}{\circ} \mathcal{K}: \mathrm{D}^{\mathrm{b}}(\mathcal{A} ; \mathfrak{M}) \rightarrow \mathrm{D}^{\mathrm{b}}(\mathcal{B} ; \mathfrak{N}), \quad \mathcal{M} \mapsto \mathbb{D} \pi_{2 *}\left(\mathbb{D} \pi_{1}^{*} \mathcal{M} \stackrel{\mathbb{D}}{\otimes} \mathcal{K}\right) .
$$

To $F \in \mathrm{D}^{\mathrm{b}}\left(\mathfrak{M}_{\mathcal{A}} \circledast_{\mathbb{C}} \mathfrak{M}\right)$ one associates the objects of $\mathrm{D}^{\mathrm{b}}(\mathcal{A} ; \mathfrak{M})$ defined by

$$
\mathcal{C}^{\natural}(F)= \begin{cases}F \otimes \mathcal{O}_{\mathcal{A}} & \text { for } \emptyset=\omega, \\ F \stackrel{\mathrm{w}}{\otimes} \mathcal{O}_{\mathcal{A}} & \text { for } \emptyset=\infty, \\ T \mathcal{H o m}\left(F^{\prime}, \mathcal{O}_{\mathcal{A}}\right) & \text { for } \underline{\natural}=-\infty, \\ R \mathcal{H o m}\left(F^{\prime}, \mathcal{O}_{\mathcal{A}}\right) & \text { for } \emptyset=-\omega,\end{cases}
$$

where $F^{\prime}=R \mathcal{H}$ om $\left(F, \mathbb{C}_{X}\right)$, and THom and $\stackrel{\text { w }}{\otimes}$ are the functors of formal and temperate cohomology of [17, 23]. (One checks that the construction in [23] of the functors of formal and temperate cohomology, starting from exact functors defined on the underlying real analytic manifolds, extends to the twisted case.) Hence, for $\natural= \pm \infty$ we have to assume that $F$ is $\mathbb{R}$-constructible.

Let $\mathcal{M} \in \mathrm{D}^{\mathrm{b}}(\mathcal{A} ; \mathfrak{M}), \mathcal{K} \in \mathrm{D}^{\mathrm{b}}\left(\mathcal{A}^{\sharp-1}\right.$ 团 $\left.\mathcal{B} ; \mathfrak{M}^{\circledast-1} \circledast \mathfrak{N}\right)$, and $G \in \mathrm{D}^{\mathrm{b}}\left(\mathfrak{M}_{\mathcal{B}} \circledast_{\mathbb{C}} \mathfrak{N}\right)$. Consider the solution complex $K=R \mathcal{H o m}{ }_{\mathcal{A}^{\sharp-1} \text { 团B }}\left(\mathcal{K}, \mathcal{O}_{\mathcal{A}^{\sharp-1} \text { 团B }}\right)$ of $\mathcal{K}$, which is an object of $\mathrm{D}^{\mathrm{b}}\left(\left(\mathfrak{M}_{\mathcal{A}} \circledast_{\mathbb{C}} \mathfrak{M}\right) \circledast\left(\mathfrak{M}_{\mathcal{B}} \circledast_{\mathbb{C}} \mathfrak{N}\right)^{\circledast-1}\right)$.

Theorem 4.19. With the above notations, assume that $\mathcal{M}$ is coherent, and $\mathcal{K}$ is regular holonomic, so that $K$ is $\mathbb{C}$-constructible. If $\square= \pm \omega$, assume that $\pi_{2}$ is proper on $\operatorname{supp}(\mathcal{K})$, and that $\operatorname{char}(\mathcal{K}) \cap\left(T^{*} X \times T_{Y}^{*} Y\right)$ is contained in the zerosection of $T^{*}(X \times Y)$. If $\downarrow= \pm \infty$, assume instead that $G$ is $\mathbb{R}$-constructible. Then, there is an isomorphism in $\mathrm{D}^{\mathrm{b}}(\mathbb{C})$

$$
\operatorname{RHom}_{\mathcal{A}}\left(\mathcal{M}, \mathcal{C}^{ \pm \natural}(K \circ G)\right)\left[d_{X}\right] \simeq \operatorname{RHom}_{\mathcal{B}}\left(\mathcal{M} \stackrel{\mathbb{D}}{\circ} \mathcal{K}, \mathcal{C}^{ \pm \natural}(G)\right)
$$

where $\left[d_{X}\right]$ denotes the shift by the complex dimension of $X$.

We do not give here the proof, which follows the same lines as the one for the non-twisted case given in [10, 23]. 


\section{References}

[1] H. Bass, Algebraic K-theory, Benjamin (1968)

[2] K. Behrend, Localization and Gromov-Witten invariants, in: Quantum cohomology (CIME, Cetraro, 1997), Lecture Notes in Math. 1776, Springer (2002), 3-38.

[3] A. Beilinson and J. Bernstein, Localisation de g-modules, C. R. Acad. Sci. Paris Sér. I Math. 292 no. 1 (1981), 15-18.

[4] _ A proof of Jantzen conjectures, in: I. M. Gelfand Seminar, Adv. Soviet Math. 16 part 1, Amer. Math. Soc. (1993), 1-50.

[5] L. Breen, On the classification of 2-gerbes and 2-stacks, Astérisque 225 (1994)

[6] L. Breen and W. Messing, Differential geometry of gerbes, e-print (2001), arXiv:math.AG/0106083.

[7] J.-L. Brylinski, Loop Spaces, Characteristic Classes and Geometric Quantization, Progress in Math. 107 (1993), Birkhäuser.

[8] A.H. Caldararu, Nonfine moduli spaces of sheaves on $K 3$ surfaces, Int. Math. Res. Not. 20 (2002), 1027-1056.

[9] A. D'Agnolo and P. Polesello, Stacks of modules over algebroids, in preparation.

[10] A. D'Agnolo and P. Schapira, Leray's quantization of projective duality, Duke Math. J. 84 no. 2 (1996), 453-496.

[11] R. Donagi and T. Pantev, Torus fibrations, gerbes, and duality, e-print (2003), arXiv:math.AG/0306213.

[12] C. Faith, Algebra. I. Rings, modules, and categories, Grundlehren der Mathematischen Wissenschaften 190, Springer (1981).

[13] O. Gabber, Letter to Bogomolov (1988).

[14] J. Giraud, Cohomologie non abelienne, Grundlehren der Math. Wiss. 179, Springer (1971)

[15] N.J. Hitchin, Lectures on Special Lagrangian Submanifolds Lectures given at the ICTP School on differential geometry, e-print (1999), arXiv:math.DG/9907034.

[16] A. Kapustin, D-branes in a topologically nontrivial B-fields, Adv. Theor. Math. Phys. 4 no. 1 (2000), 127-154.

[17] M. Kashiwara, The Riemann-Hilbert problem for holonomic systems, Publ. Res. Inst. Math. Sci. 20 no. 2 (1984), 319-365.

[18] $\_$, Representation theory and $\mathcal{D}$-modules on flag varieties, in: Orbites unipotentes et représentations, III, Astérisque 173-174 (1989), 55-109.

[19] - Algebraic study of systems of partial differential equations, Kashiwara's Master's Thesis (Tokyo University 1970), translated from the Japanese by A. D'Agnolo and J.-P. Schneiders, Mém. Soc. Math. France 63 (1995).

[20] Quantization of contact manifolds, Publ. Res. Inst. Math. Sci. 32 no. 1 (1996), 1-7.

[21] _ D-modules and microlocal calculus, translated from the 2000 Japanese original by M. Saito, Transl. of Math. Monographs 217, A.M.S. (2003). 
[22] M. Kashiwara and P. Schapira, Sheaves on manifolds, Grundlehren der Mathematischen Wissenschaften 292, Springer (1990).

[23] Moderate and formal cohomology associated with constructible sheaves, Mém. Soc. Math. France 64 (1996).

[24] Ind-sheaves, Astérisque 271 (2001).

[25] Categories and sheaves, preliminary version of a book in preparation $(8 / 2002)$.

[26] M. Kashiwara and W. Schmid, Equivariant derived category and representation of semisimple Lie groups, preliminary version (1994). See the announcement: Quasiequivariant $\mathcal{D}$-modules, equivariant derived category, and representations of reductive Lie groups, in: Lie theory and geometry, Birkhäuser (1994), 457-488.

[27] M. Kashiwara and T. Tanisaki, Kazhdan-Lusztig conjecture for affine Lie algebras with negative level. II. Nonintegral case, Duke Math. J. 84 no. 3 (1996), 771-813.

[28] M. Kontsevich, Deformation quantization of algebraic varieties. in: EuroConférence Moshé Flato, Part III (Dijon, 2000). Lett. Math. Phys. 56 no. 3 (2001), 271-294.

[29] G. Laumon and L. Moret-Bailly, Champs algébriques, Ergebnisse der Mathematik und ihrer Grenzgebiete 39, Springer (2000).

[30] P. Polesello and P. Schapira, Stacks of quantization-deformation modules on complex symplectic manifolds, e-print (2003), arXiv:math.AG/0305171.

[31] M. Murray, Bundle gerbes, J. London Math. Soc. 54 (1996), 403-416.

[32] D. Stevenson, The geometry of bundle gerbes, Ph.D. Thesis (Adelaide Univ. 2000), e-print (2000), arXiv:math.DG/0004117.

[33] R. Street, Categorical structures, in: Handbook of algebra 1, North-Holland (1996), 529-577.

[SGA1] A. Grothendieck, Revêtements étales et groupe fondamental. With contributions by M. Raynaud. Lecture Notes in Mathematics 224, Springer (1971).

[SGA4] M. Artin, A. Grothendieck, and J.L. Verdier, Théorie des topos et cohomologie étale des schémas. Tome 1: Théorie des topos. Lecture Notes in Mathematics 269, Springer (1972).

A.D'A.: Dipartimento di Matematica Pura ed Applicata; Università di Padova; Via G. Belzoni, 7; 35131 Padova; Italy

P.P.: Dipartimento di Matematica Pura ed Applicata; Università di Padova; Via G. Belzoni, 7; 35131 Padova; Italy; and: Analyse Algébrique; Institut de Mathématiques; 175, rue du Chevaleret; 75013 Paris; France

Email: dagnolo@math.unipd.it,pietro@math.jussieu.fr 